\title{
K-MACE Clustering for Gaussian Clusters
}

\author{
by \\ Edward Wyndel Nidoy \\ Bachelor of Engineering, Ryerson University, 2015
}

A thesis

presented to Ryerson University

in partial fulfillment of the

requirements for the degree of

Master of Applied Science

in the Program of

Electrical and Computer Engineering

Toronto, Ontario, Canada, 2017

(C)Edward Wyndel Nidoy 2017 


\section{AUTHOR'S DECLARATION FOR ELECTRONIC SUBMISSION OF A THESIS}

I hereby declare that I am the sole author of this thesis. This is a true copy of the thesis, including any required final revisions, as accepted by my examiners.

I authorize Ryerson University to lend this thesis to other institutions or individuals for the purpose of scholarly research.

I further authorize Ryerson University to reproduce this thesis by photocopying or by other means, in total or in part, at the request of other institutions or individuals for the purpose of scholarly research.

I understand that my dissertation may be made electronically available to the public. 


\title{
k-MACE Clustering for Gaussian Clusters
}

Master of Applied Science 2017

Edward Wyndel Nidoy

Electrical and Computer Engineering

Ryerson University

\begin{abstract}
Conventional clustering approaches require a preprocessing step that estimates the correct number of cluster prior to the cluster center allocation step. In these approaches, the preprocessing step minimizes one objective function while the second step concentrates on optimization of another objective function. Inspired by MACE-means, we use a single objective function to simultaneously estimate the Correct Number of Cluster (CNC) and acquire the cluster centers. Similarly, we use the Average Central Error (ACE) as our cost function. The proposed method, denoted by k-minimum ACE (k-MACE), improves MACE-means by rigorous calculation of probabilistic estimate of ACE. While MACEmeans (Minimum ACE) only concentrates on Independent Indentically Distributed (IID) clusters, $\mathrm{k}-\mathrm{MACE}$ is a solution for Gaussian clusters with any covariance structure. Simulation results show superiority of $\mathrm{k}$ - MACE over MACE means and over conventional clustering methods such as G-means, DBSCAN, and validity indices methods such as Calinkski Harabaz, Silhoutte, and gap index. Performance is evaluated in terms of
\end{abstract}


accuracy of CNC estimation, adjusted random index and normalized variation information. 


\section{Acknowledgements}

First and foremost, I would like to express my sincerest gratitude to Dr. Soosan Beheshti for all her support through my MASc study and other research endeavors. Her immense knowledge, patience and motivation has been a vital part of this work and had greatly helped me in the research and writing of this thesis. I am truly grateful for having such an amazing mentor.

I would also like to thank the members of my thesis committee: Dr. Reza Sedaghat, Dr. Lian Zhao, and Dr. Alagan Anpalagan as well as Dr. Assad Sahabelam, for their insightful comments and encouragement that inspired me to widen my research from various perspectives.

I also want to thank my fellow lab-mates in the Signal and Information Processing Lab as well as my friends at Ryerson University for the stimulating discussions.

Last but not the least, I would like to thank my family: my brothers, and my aunt and uncle who serve as our guardian. Their support through out all these years made this accomplishment possible. 


\section{Dedication}

To the loving memory of my mom and dad. 


\section{Contents}

Declaration ............................... ii

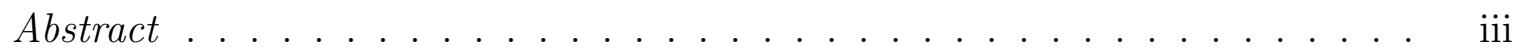

Acknowledgements ......................... . . v

Dedication ................................... vi

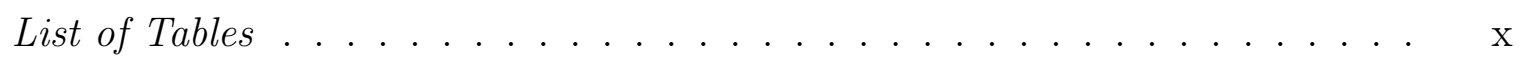

List of Figures . . . . . . . . . . . . . . . . . . . xi

List of Abbreviations . . . . . . . . . . . . . . . . . xiv

$\begin{array}{lll}1 & \text { Introduction } & 1\end{array}$

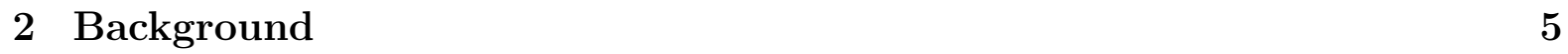

2.1 Partitional Clustering Methods $\ldots \ldots \ldots \ldots$

2.1 .1 K-means . . . . . . . . . . . . . . . . . 6

$2.1 .2 \quad$ Fuzzy-C means $\ldots \ldots \ldots \ldots \ldots \ldots \ldots$

$2.2 \quad$ Index Validity Methods $\ldots \ldots \ldots$

$2.2 .1 \quad$ Xie-Beni Index $\ldots \ldots \ldots \ldots \ldots$

2.2 .2 Silhouette Index . . . . . . . . . . . . . . . . . . 11

2.2 .3 Dunn Index $\ldots \ldots \ldots \ldots$

2.2 .4 Calinski-Harabasz index $\ldots \ldots \ldots \ldots \ldots$

2.2 .5 Davies-Bouldin Index . . . . . . . . . . . . . . . . . . . 15 
$2.2 .6 \quad$ Several Validity Index Behavior With Respect to k . . . . . . . . 16

2.3 Information-Theoretic Approach to Signal Denoising and Best Basis Selection 20 2.3 .1 Estimation of Reconstruction Error from Data Error . . . . . . . 23

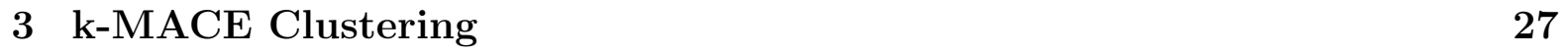

3.1 Preliminaries and Notations . . . . . . . . . . . . . . . . . . . . . . . . 28

3.1 .1 Data Model . . . . . . . . . . . . . . . . . . . . . . . . . . . . . 28

$3.1 .2 \quad$ Clustering the Available Data Into $m$ Clusters . . . . . . . . . . . 32

3.1 .3 Average Central Error . . . . . . . . . . . . . . . . . . . . . . 32

3.1 .4 Data Error . . . . . . . . . . . . . . . . . . . . . . 34

3.2 k-MACE Approach . . . . . . . . . . . . . . . . . . . . . . . . . . . . . 35

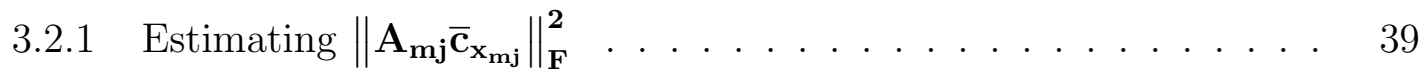

$3.3 \quad$ Estimating $\bar{\wedge}_{\mathbf{x}_{\mathbf{i}}}$. . . . . . . . . . . . . . . . . . . . . . . . . . . 42

3.3 .1 Obtaining $\hat{\mathbf{m}}^{0} \ldots \ldots \ldots \ldots$. . . . . . . . . . . . . . . 45

3.3 .2 Why Use GMM for Estimation of Covariances . . . . . . . . . . . 46

3.4 Computational Complexity of k-MACE . . . . . . . . . . . . . . . . . . . 49

4 k-MACE Clustering $\quad 51$

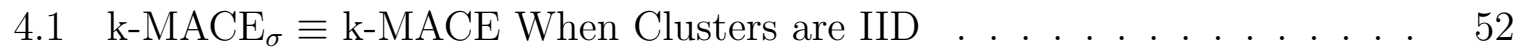

4.1 .1 Average Central Error . . . . . . . . . . . . . . . . . . 53

4.1 .2 Data Error . . . . . . . . . . . . . . . . . 54

$4.1 .3 \quad$ Estimating $\sigma_{\mathrm{w}}^{2} \ldots \ldots \ldots \ldots \ldots \ldots \ldots$

4.2 Computational Analysis of $\mathrm{k}-\mathrm{MACE}_{\sigma}$. . . . . . . . . . . . . . 56

4.3 An Example of k-MACE and $\mathrm{k}-M A C E_{\sigma}$ on Clusters That Are IID . . . . 57

4.4 Smart k-MACE ${ }_{\sigma}$ Validation $\ldots \ldots \ldots$

4.5 Smart k-MACE ${ }_{\sigma}$ Clustering . . . . . . . . . . . . . . . . . 60 
4.5 .1 Indentifying Touching Clusters $\ldots \ldots \ldots \ldots \ldots$

4.5 .2 Unimodality Test for Touching Clusters . . . . . . . . . . . 62

$\begin{array}{lll}5 & \text { Simulations and Result } & 67\end{array}$

$5.1 \quad$ Artificial Data Sets . . . . . . . . . . . . . . . . . . . . 67

5.2 Real World Bench Mark Data Sets . . . . . . . . . . . . . . 75

6 Conclusion and Future Work $\quad 81$

\begin{tabular}{lr}
\hline Appendices & 84
\end{tabular}

\begin{tabular}{lr}
\hline A Average Central Error & 86
\end{tabular}

A.1 Expected Value of ACE $\ldots \ldots \ldots$

A.2 Variance of $\mathrm{ACE} \ldots \ldots \ldots \ldots$

A.3 Average Central Error When $\bar{\Sigma}_{X_{i}}=\sigma_{w}^{2} I_{d x d} \ldots \ldots \ldots \ldots$. . . . . 89

\begin{tabular}{ll}
\hline B Data Error & 91
\end{tabular}

B.1 Expected Value of Data Error . . . . . . . . . . . . . . . . . . . . 92

B.2 Variance of Data Error . . . . . . . . . . . . . . . . . . . . . . . . 92

B.3 Average Central Error When $\overline{\boldsymbol{\Sigma}}_{\mathbf{X}_{\mathbf{i}}}=\sigma_{\mathbf{w}}^{2} \mathbf{I}_{\mathbf{d x d}} \ldots \ldots \ldots \ldots . \ldots . \ldots 94$ 


\section{List of Tables}

$2.1 \quad$ Advantages and disadvantages of k-means and FCM . . . . . . . . . . . . 9

3.1 Table of symbols used in $\mathrm{k}-\mathrm{MACE}$ and $\mathrm{k}-\mathrm{MACE}_{\sigma}$ formulations Part 129

3.2 Table of symbols used in $\mathrm{k}-\mathrm{MACE}$ and $\mathrm{k}-\mathrm{MACE}_{\sigma}$ formulations Part 230

$5.1 \quad$ Artificial data set. Result is generated from the average of 50 runs. Results

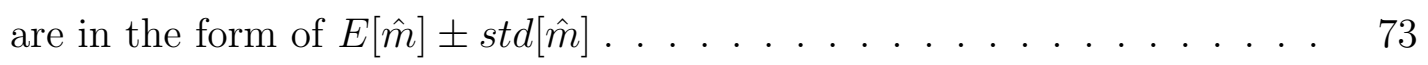

5.2 Real data set 1. Result is generated from the average of 50 runs. . . . . . 78

5.3 Real data set 2. Result is generated from the average of 50 runs. . . . . . 79

5.4 Real data set 3. Result is generated from the average of 50 runs. . . . . . 80 


\section{List of Figures}

$2.1 \quad$ Validity index on data set with IID clusters, uniform variance and uniform proximity. . . . . . . . . . . . . . . . . . 16

$2.2 \quad$ Validity index on data set with IID clusters, unique variance and inconsistent proximity. . . . . . . . . . . . . . . . . . . . . . . . . 17

$2.3 \quad$ Validity index on data set with general Gaussian clusters, unique distribution and uniform proximity. . . . . . . . . . . . . . . . . . . . . . . . . 17

2.4 Validity index on data set with general Gaussian clusters, unique distribution and inconsistent proximity. . . . . . . . . . . . . . . . . . . . . . . . 18

2.5 Validity index on Aggregation data set [29] . . . . . . . . . . . . . . . . . 18

$2.6 \quad$ Validity index on R15 data set $[30] \ldots$. . . . . . . . . . . . . . . . . . . . 19

$2.7 \quad$ Validity index on D31 data set $[30][\ldots \ldots$. . . . . . . . . . . . . . 19

$3.1 \quad$ Example of clustering solution and its corresponding estimated centers $\left(\hat{c}_{m j}\right)$ as well as the true cluster centers $\left(\bar{c}_{j}\right) \ldots \ldots$. . . . . . . . . . 33

3.2 Example behavior of $Z_{s_{m}}$ and $Y_{s_{m}}$ on general Gaussian clusters . . . . . . 36

3.3 Expected value and variance of $Z_{s_{m}}$ for a range of $m$ derived from data set depicted on Figure $3.2-\mathrm{a}$. . . . . . . . . . . . . . . . . . . . . . . . . 38

$3.4 \quad$ Expected value and variance of $Y_{s_{m}}$ for a range of $m$ derived from data set S1 depicted on figure $1 \mathrm{a}$. . . . . . . . . . . . . . . . . . . 40 
3.5 Behavior of $Z_{s_{m}}$ and $m_{l}$ and its derived bounds derived from data set

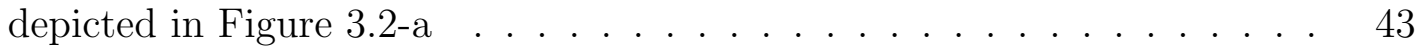

$3.6 \quad$ Clustering result when $m>\bar{m}$ (here, $\bar{m}=2, d=2, N=21$ ) . . . . . . . . 43

3.7 Clustering solution: GMM vs K-means . . . . . . . . . . . . . . . . . . . 47

3.8 Behavior of $Z_{s_{m}}$ and $Y_{s_{m}}$ : GMM vs K-means . . . . . . . . . . . . . . 48

$4.1 \quad$ Example behavior of $Z_{s_{m}}$ and $Y_{s_{m}}$ on IID clusters . . . . . . . . . 52

4.2 Example Clustering Solution: $\mathrm{k}-\mathrm{MACE}$ vs $\mathrm{k}-\mathrm{MACE}_{\sigma}$ on IID clusters 58

4.3 k-MACE on D31 data set . . . . . . . . . . . . . . . . . . . . . . . . . . 59

4.4 Example Clustering Solution: $\mathrm{k}$ - MACE vs k - MACE ${ }_{\sigma}$ on general Gaussian clusters. . . . . . . . . . . . . . . . . . . . . 61

4.5 Touching clusters formed by splitting of a unimodal cluster . . . . . . . . 64

4.6 Two generally distributed touching clusters . . . . . . . . . . . . . . . . . 64

4.7 Two touching IID clusters $\ldots \ldots \ldots$. . . . . . . . . . . . . . 64

4.8 Clusters connected through a neck . . . . . . . . . . . . . . . . 64

$5.1 \quad$ IID clusters, uniform variance and uniform proximity (data set characteristic: $\bar{m}=9, d=2, N=900$ (result: $\left.\hat{m}_{\mathrm{k}-\mathrm{MACE}}=9, \hat{m}_{\mathrm{k}-\mathrm{MACE}_{\sigma}}=9, \hat{m}_{\mathrm{MM}}=9\right) \quad 68$

5.2 IID clusters, unique variance, unique proximity (data set characteristic: $\bar{m}=5, d=2, N=1731$ ) (result: $\hat{m}_{\mathrm{k}-\mathrm{MACE}}=4, \hat{m}_{\mathrm{k}-\mathrm{MACE}_{\sigma}}=9, \hat{m}_{\mathrm{mm}}=6$ ) $\quad 69$

5.3 General Gaussian, unique covariance, uniform proximity (data set characteristic: $\bar{m}=9, d=2, N=900$ ) (result: $\hat{m}_{\mathrm{k}-\mathrm{MACE}}=9, \hat{m}_{\mathrm{k}-\mathrm{MACE}_{\sigma}}=14, \hat{m}_{\mathrm{mm}}=13$ ) 69

5.4 General Gaussian, Unique covariance, varying proximity (data set characteristic: $\bar{m}=9, d=2, N=900)\left(\right.$ result: $\left.\hat{m}_{\mathrm{k}-\mathrm{MACE}}=9, \hat{m}_{\mathrm{k}-\mathrm{MACE}_{\sigma}}=13, \hat{m}_{\mathrm{mm}}=15\right)$

$5.5 \quad$ Aggregation data set (data set characteristic: $\bar{m}=7, d=2, N=787$ ) (result: $\left.\left.\hat{m}_{\mathrm{k}-\mathrm{MACE}}=11, \hat{m}_{\mathrm{k}-\mathrm{MACE}_{\sigma}}=14, \hat{m}_{\mathrm{mm}}=23\right)\right] \ldots . . . . . \quad 71$ 
5.6 R15 data set (data set characteristic: $\bar{m}=15, d=2, N=600$ ) (result: $\left.\hat{m}_{\mathrm{k}-\mathrm{MACE}}=15, \hat{m}_{\mathrm{k}-\mathrm{MACE}_{\sigma}}=15, \hat{m}_{\mathrm{mm}}=15\right) \ldots \ldots \ldots 71$

5.7 D31 data set (data set characteristic: $\bar{m}=31, d=2, N=3100$ ) (result: $\left.\hat{m}_{\mathrm{k}-\mathrm{MACE}}=22, \hat{m}_{\mathrm{k}-\mathrm{MACE}}=31, \hat{m}_{\mathrm{mm}}=31\right) \ldots \ldots \ldots . \ldots . \ldots 72$

5.8 Dimension reduced abalone data set $\ldots \ldots \ldots$. . . . . . . . . 77 


\section{List of Abbreviations}

CNC ........ Correct Number of Cluster

ACE....$\ldots$. Average Central Error

IID .......... Independent Indentically Distributed

GMM $\ldots . . .$. Gaussian Mixuture Model

NVI ......... Normalized Variation Index

ARI ......... Adjusted Random Index

BIC .......... Bayesian Information Criterion

AD $\ldots \ldots \ldots \ldots$ Anderson-Darling

WGSS ....... Within-Group Scatter

BGSS ....... Between Group Scatter

WTP ........ Water Treatment Plant

WDBC ....... Wisconsin Diagnostic Breast Cancer 



\section{Chapter 1}

\section{Introduction}

Clustering is the process of grouping observed data samples based on their similarities and dissimilarities such that objects from the same group exhibits more similar properties compared to objects from a different group. Clustering has a wide variety of application in the fields of engineering, statistics, academics, image processing, bioinformatics, business, medicine, psychiatry, psychology, geography and computer science, to name a few [1. In adaptive image denoising, clustering can be utilized to identify patches of an image that exhibits similar geometric and photometric characteristic. This improves the exploitation of image redundancy since identification of similar patches are not limited by a search window [4. In market research, clustering is used to group customers based on their Internet search results, be it in the form of text, image or audio data. Using such information, product recommendations can be made making it beneficial for both the customer and the seller. In the field of academics, a class's academic performance can be monitored by grouping students based on their performance levels with the use of clustering [2]. In health sciences, clustering was used to determine underlying characteristic of cancerous data. Known cancerous and non cancerous data is mixed into one data 
set and several clustering methods were employed to partition this data set. It was found that non-linear clustering methods were able to provide an accurate partition between the cancerous and the non-cancerous data concluding the non-linear nature of cancerous data 3$]$.

Clustering algorithm can be sub-categorized by the following [5]: partitional clustering and hierarchical clustering. Partitional clustering attempts to partition the data into non-overlapping clusters and evaluate such partition based on an objective function. Hierarchical clustering algorithm is done by repeatedly dividing larger clusters into smaller ones or merging smaller clusters into larger ones. By doing so, a hierarchy of clusters, known as a dendogram, is produced. Depending on the level at which the dendogram is cut, different clustering solution can be obtained. A priori assumption of the cluster structure plays an important role in clustering since it determines which clustering algorithms are more suitable for a specific data set. For example, data sets that follow an arbitrary shape are better dealt with using hierarchical clustering as oppose to partitional clustering. Partional clustering is more applicable if the cluster distribution is known (i.e Gaussian, uniform, etc).

Majority of partitional clustering method requires a priori knowledge of the CNC. Overestimating the number of clusters typically result into inappropriate separations from one good compact cluster into smaller clusters that are close to each other. Underestimating the number of clusters, on the other hand, results into combining compact clusters to form a loose cluster. In real life applications, $\mathrm{CNC}$ is not available and an optimum estimation is difficult to be intuitively determined beforehand.

Pioneer methods of estimation of correct number of clusters involves formulation of validity indexes. Validity indexes provide a quantitative measurement of the wellness of the clustering result based on the estimated number of clusters. Examples of these validity 
indexes are Xie-Beni index [7, Silhouette index 88, Dunn index 99, Calinski-Harabasz index 10, Davies-Bouldin index 11, Krzanowski-Lai index 12, weighted inter-to-intracluster ratio [13, Fukuyama-Sugeno index [14, the partition density 15 and the PBM index 16 just to name a few. In general, the process of these validity indexes method involves clustering the data, typically with k-means [6], into $k$ clusters in a predetermined range $k=\left[k_{\min }, k_{\min }+1, \ldots, k_{\max }\right]$. For each $k$-clustering result, validity indexes are evaluated. The $k$ value in which the validity index is optimized is chosen as the estimated correct number of clusters.

Another approach of estimating the correct number of clusters are X-means 17] and G-means 18 which are typically used as wrapper to k-means. The difference between the two is the statistical test that each method uses to asses whether or not a single cluster should be further divided. For both cases, the algorithm starts with clustering the data using k-means with $k$ amount of clusters. A statistical test is then run for each cluster in order to determine whether a cluster is already compact or if it needs to be split further. In the case of X-means, Bayesian Information Criterion (BIC) 19] is the choice of statistical test whereas in G-means, Anderson-Darling (AD) 20 is used.

Similar to the clustering methods mentioned above, MACE-means 23 clustering tackles the problem of estimating the correct number of clusters but in a probabilistic approach. MACE means algorithm is suitable when all the cluster structures are assumed to have an IID. It also serves as a wrapper to k-means clustering. In MACE means, the criterion that is being optimized as a function of $m$ is called the ACE which is the average distance between the estimated centers and the true cluster centers. The calculation of ACE requires the knowledge of the cluster's true center which is unavailable in real applications. MACE means provide a probabilistic approach, derived from 21, to estimate the expected value of the unobservable ACE using the available cluster 
compactness. MACE means shows weaknesses when data is composed of clusters that follow a general Gaussian distribution. In addition to this, MACE means only use the estimated expected value of ACE and disregard ACE's variance which affects the accuracy of ACE's estimation. 21.

Our Objectives: In this thesis, our main focus is to improve MACE-means through rigorous calculation of probabilistic bounds of ACE. We also aim to extend the applicability of MACE means by making it suitable for Gaussian clusters with any covariance structure.

Thesis Outline: This thesis is organized as follows: In Chapter 2, brief background of several widely used clustering methods as well as the data denoising technique that inspired MACE means is provided. k-minimizing Average Central Error (k - MACE) for estimating CNC for general Gaussian Clusters is proposed in Chapter 3. The role of probabilistic bounds on ACE's estimation is also introduced in Chapter 3. Chapter 4 presents a special case of $\mathrm{k}-\mathrm{MACE}$ denoted by $\mathrm{k}-\mathrm{MACE}_{\sigma}$ that is applicable when data set is composed of clusters that are IID. Smart $\mathrm{k}-\mathrm{MACE}_{\sigma}$, a method that can validate whether clusters are IID is also discussed in Chapter 4. Discussion of our simulation results is shown on Chapter 5 and finally, Chapter 6 presents concluding remarks and suggestions for future works. 


\section{Chapter 2}

\section{Background}

In this Chapter, we review some of the widely used clustering algorithms as well as the signal denoising scheme that inspired the $\mathrm{k}-\mathrm{MACE}$ clustering.

In section 2.1, we discuss the two most common partitional clustering methods which are K-means and Fuzzy C-means. We compared the performance of these two clustering scheme and highlight their strengths and weaknesses.

Validity indexes such as Xie-Beni, Silhouette, Dunn, Calinkski-Harabasz and DaviesBouldin which can be used to assess the wellness of a clustering solution is discussed in section 2.2. The role of these indexes in estimation of CNC is also discussed.

In section 2.3, we discuss a signal denoising scheme that inspired k-MACE. Signal denoising is achieved through best subspace selection and through estimation of an unobservable error.

\subsection{Partitional Clustering Methods}

Partitional clustering divides a data set into a set of disjoint clusters. Given a data set of length $N, x^{N}=\left[x_{1}, x_{2}, \ldots, x_{N}\right]$, partitional clustering algorithms constructs $k(k<N)$ 
clusters. The value $k$, number of clusters, is usually provided by the user.

It is important to note that partitional clustering works best when the clusters are distance separated and follows some distribution (i.e. Gaussian). However, it is weak against data where clusters follow an arbitrary shape. For this type of data, hierarchical clustering or spectral clustering would be more appropriate.

\subsubsection{K-means}

k-means is one of the most well-known and widely used clustering methods mainly due to its simplicity and reasonable convergence speed. The procedure aims to classify the data with a certain number of clusters $(k)$ given apriori. k-means iteratively looks for the optimum location of $k$ cluster centers, $c_{j}([j=1, \ldots, k])$, so that the following objective function is optimized:

$$
J(c)=\sum_{j=1}^{k} \sum_{x_{i} \in C_{j}}\left(\left\|x_{i}-c_{j}\right\|\right)^{2}
$$

where $c_{j}$ is the center of cluster $C_{j}, x_{i} \in C_{j}$ pertains to all elements in cluster $C_{j}, n_{j}$ is the number of elements in cluster $C_{j}$ and $\|*\|$ calculates for the Euclidean distance.

The algorithm for $k$ - means are as follows:

Consider a data set of length $N, x^{N}=\left[x_{1}, x_{2}, \ldots ., x_{N}\right]^{T}$ where $x_{i} \in R_{1 \times d}$. Each element $x_{i}$ is a vector of length $d$, and each scalar element of $x_{i}$ represents the observed feature. The goal is to partition the data samples into $k$ clusters in a way that the internal distance of a sample to its cocluster members are smaller compared to its distances to samples that belongs to a different cluster. Let $c=\left[c_{1}, \ldots, c_{k}\right]$ be the set of centers of each cluster where $c_{j} \in R_{1 \times d}$.

1. In $d$ dimensional space, randomly select the location of $k$ cluster centers, $c$.

2. Calculate the Euclidean distance between all data samples and each of the cluster 
center.

3. For each data sample $x_{i}$, find the cluster whose center is closest to $x_{i}$ and assign $x_{i}$ to that cluster.

4. Recalculate each of the cluster center by taking the mean of all data samples that belongs to that cluster.

$$
c_{j}=\frac{1}{n_{j}} \Sigma_{i=1}^{n_{j}} x_{i}
$$

5. Recalculate the distance between the new cluster centers and each data sample.

6. Reassign each sample's cluster membership based on the recalculated center.

7. If no data sample was reassigned or the maximum number of iteration is reached, then stop. If not, go back to step 4.

\subsubsection{Fuzzy-C means}

Fuzzy clustering is a clustering algorithm in which a sample is given a weighted membership to all the clusters. This alleviates one of the weaknesses of k-means that comes from exclusive assignment. In Fuzzy C means, the clustering solution is achieved by optimization of the following objective function:

$$
J(c)=\sum_{i=1}^{N} \sum_{j=1}^{k} \mu_{i j}\left(\left\|x_{i}-c_{j}\right\|\right)^{2}
$$

where $k$ is the number of clusters, $u_{i j}$ is the degree of membership of $x_{i}$ to the $j^{t h}$ cluster. (Note that $\sum_{j=1}^{m} \mu_{i j}=1$ ).

Similar to k-means, fuzzy-C means is also carried out through an iterative optimization of the objective function in 2.3. The step-by-step process is described below: 
Consider a data set of length $N, x^{N}$ to be clustered into $k$ groups. We let $x_{i}$ be an element of the data set and $c_{j}$ be the center of the $j^{\text {th }}$ cluster.

1. Randomly initialize cluster membership $U=\left[\mu_{i j}\right]$, an $(N \times k)$ matrix where each column represents the cluster membership of each row element.

2. Based on the cluster membership $U$, calculate the $k$ cluster center vector $c_{1}, \ldots, c_{k}$.

$$
c_{j}=\frac{\sum_{i=1}^{N} \mu_{i j}^{m} x_{i}}{\sum_{i=1}^{N} \mu_{i j}}
$$

3. Update the cluster membership matrix $U$ based on the cluster centers $c$

$$
\mu_{i j}=\frac{1}{\sum_{j=1}^{k}\left(\frac{\left\|x_{i}-c_{j}\right\|}{\left\|x_{i}-c_{k}\right\|}\right)^{\frac{2}{m-1}}}
$$

4. If the maximum number of iteration is reached or if there is no significant change from the recalculated cluster membership $U$, then the algorithm is finished 1 Otherwise return to step 2 .

Table 2.1 compares the two partitonal clustering algorithm discussed previously and highlights their advantages and disadvantages.

\footnotetext{
${ }^{1}$ insignificant change of cluser membership matrix $U$ can be detected by the following: $\left\|U_{\text {old }}-U_{\text {new }}\right\|<a$ where $a$ is a small threshold value.
} 
Table 2.1: Advantages and disadvantages of k-means and FCM

\begin{tabular}{|c|c|c|}
\hline & Advantages & Disadvantages \\
\hline k-means & $\begin{array}{l}\text { (1) Fast, robust and easier to understand. } \\
\text { (2) Relatively efficient: } O(t k N d) \text {, where } t \text { is the } \\
\text { maximum number of iterations. } \\
\text { (3) Gives best result when data set are distinct or well } \\
\text { separated from each other. }\end{array}$ & $\begin{array}{l}\text { (1) The learning algorithm requires apriori specification } \\
\text { of the number of,cluster centers. } \\
\text { (2) The use of,Exclusive Assignment - If,there are two highly } \\
\text { overlapping data then k-means will not be able to resolve that } \\
\text { there are two clusters. } \\
\text { (3) Euclidean distance measures can unequally weight } \\
\text { underlying factors. } \\
\text { (4) The learning algorithm provides the local optima of the } \\
\text { squared error function. } \\
\text { (5) Sensitive to the initial value of the cluster centers which } \\
\text { are chosen randomly. } \\
\text { (6) Applicable only when mean is defined i.e. fails for } \\
\text { categorical data. } \\
\text { (7) Unable to handle noisy data and outliers. } \\
\text { (8) Algorithm fails for non-linear data set. }\end{array}$ \\
\hline FCM & $\begin{array}{l}\text { (1) FCM tries to deal with the problem where points are } \\
\text { somewhat equally close to two separate clusters. } \\
\text { (2) Relatively efficient: } O(t k N d) \text {, where } t \text { is the } \\
\text { maximum number of iterations. } \\
\text { (3) In step } 2 \text { of FCM, the recalculation of the new center } \\
\text { involves all data samples as opposed to just data samples } \\
\text { that belongs to a specific cluster (case of k-means). } \\
\text { As a result, the cluster centers tends to converge to the } \\
\text { optimal solution faster. }\end{array}$ & $\begin{array}{l}\text { (1) Requires apriori assumption of the number of clusters. } \\
\text { (2) Euclidean distance measures can unequally weight } \\
\text { underlying factors. } \\
\text { (3) Less sensitive to the random initialization of } U^{(0)} \\
\text { compared to k-means. } \\
\text { (4) Requires more complex computation compared to k-means. } \\
\text { (5) With lower value for } m \text { (degree of fuzziness), we can } \\
\text { get better result but at the expense of more number of } \\
\text { iteration. } \\
\text { (6) Provides inconsistent clustering solution when dealing } \\
\text { with overlapping clusters }\end{array}$ \\
\hline
\end{tabular}




\section{$2.2 \quad$ Index Validity Methods}

Pioneer methods of estimation of correct number of clusters involves formulation of validity indexes. In order to find the best partition of the data, one usually executes a clustering algorithm with different values of number of clusters $k$ from a given range $\left(k_{\min } \leq k \leq K_{\max }\right)$. Most common choice of the clustering algorithm is the k-means algorithm. One then computes a validity index $Q_{k}$ from the clustering result.. The $k$ value that leads to the partition with the most optimized $Q_{k}$ is then identified as the estimate of CNC. Few examples of these validity indexes are: Xie-Beni index [7, Sillhoutte index 8, Dunn index 9, Calinski-Harabasz index [10, and Davies-Bouldin index 11.

\subsubsection{Xie-Beni Index}

The Xie-Beni index is defined as the quotient between the average Within-Group Scatter (WGSS) and the minimum distance between two clusters. In this case, the distance between two clusters, $C_{i}$ and $C_{j}$ is given by the distance between their respective centers, $c_{i}$ and $c_{j}$. The Xie-Beni index can be written like the following:

$$
X B_{k}=\frac{1}{N} \frac{W G S S}{\min _{i, j \leq k, i \neq j} d\left(c_{i}, c_{j}\right)}
$$

and

$$
W G S S=\sum_{j=1}^{k} \sum_{i=1}^{n_{j}}\left\|x_{j}^{i}-c_{j}\right\|^{2}
$$

the notation $x_{j}^{i}$ points to the $i^{\text {th }}$ element of the $j^{\text {th }}$ cluster and $c_{j}$ is the center of the $j^{\text {th }}$ cluster. The denominator term of 2.6 is the smallest distance from the distances between all cluster centers.

For Xie-Beni index, the numerator term is a decreasing function of $k$ (the number of 
clusters). Overestimation of the number of cluster is punished by the denominator term. If one compact cluster is divided into two clusters that are more compact but not well separated, the denominator term of the Xie-Beni index will decrease making the overall validity index bigger. This being said, the optimum number of cluster is estimated by finding the $k$ in that minimizes the Xie-Beni index.

$$
\hat{k}=\arg \min _{k}\left(X B_{k}\right)
$$

\subsubsection{Silhouette Index}

Consider for each element $x_{i} \in x^{N}$, its within-cluster average distance $a\left(x_{i}\right)$ which is the average distance of $x_{i}$ to all the other elements of the cluster that $x_{i}$ belongs to. For example, given $x_{i} \in C_{j}$, then

$$
a\left(x_{i}\right)=\frac{1}{n_{j}-1} \sum_{x_{k} \in C_{j}, i \neq k} d\left(x_{i}, x_{k}\right)
$$

where $d\left(x_{i}, x_{k}\right)$ denotes the distance between elements $x_{i}$ and $x_{k}$ and $n_{j}$ is the number of elements in cluster $C_{j}$.

For each element $x_{i}$ we also evaluate its distance to other elements that belongs to a different cluster. For example, element $x_{i}$ distance to cluster $C_{j^{\prime}}$, given that $x_{i} \notin C_{j^{\prime}}$, is given by

$$
\delta\left(x_{i}, C_{j^{\prime}}\right)=\frac{1}{n_{j^{\prime}}} \sum_{x_{k} \in C_{j^{\prime}}} d\left(x_{i}, x_{k}\right)
$$

where $n_{j^{\prime}}$ is the number of elements in cluster $C_{j^{\prime}}$.

Letting $b\left(x_{i}\right)$ denote the smallest of the average distances of $x_{i}$ to all other clusters 
(Noting $C_{j}$ as the cluster in which element $x_{i}$ belongs to),

$$
b\left(x_{i}\right)=\min _{C_{j} \neq C_{j^{\prime}}} \delta\left(x_{i}, C_{j^{\prime}}\right)
$$

For each element $x_{i}$, one then forms the ratio $s\left(x_{i}\right)$ where

$$
s\left(x_{i}\right)=\frac{b\left(x_{i}\right)-a\left(x_{i}\right)}{\max \left(a\left(x_{i}\right), b\left(x_{i}\right)\right)}
$$

$s\left(x_{i}\right)$ is also known as the silhouette width of the element $x_{i}$. It is a quantity between -1 and 1 . A value near -1 indicates that $x_{i}$ should be a member of another cluster and a value of +1 indicates that element $x_{i}$ rightfully belongs to its current cluster.

The cluster mean silhouette is the average of all its elements silhouette width. This is denoted by $S\left(C_{j}\right)$ where

$$
S\left(C_{j}\right)=\frac{1}{n_{j}} \sum_{x_{i} \in C_{j}} s\left(x_{i}\right)
$$

Finally, the overall silhouette index is the average of the cluster mean silhouette

$$
S I L_{k}=\frac{1}{k} \sum_{j=1}^{k} S\left(C_{j}\right)
$$

Minimizing the overall silhouette will require minimizing the individual silhouette for every single element $x_{i} \in x^{N}$. If the number of cluster is underestimated, loose clusters will be formed and when such thing happens, the term $a\left(x_{i}\right)$ will be large and at the same time, the term $b\left(x_{i}\right)$ will be smaller, therefore, making $s\left(x_{i}\right)$ have a negative value. On the other hand, when the number of clusters is overestimated, it will result into clusters that are not well-separated from each other. The elements that are located on the boundary between two touching clusters will have $s\left(x_{i}\right) \approx-1$ and will therefore make the overall 
silhouette smaller. The number of cluster can be estimated by maximizing the silhouette index,

$$
\hat{k}=\arg \min _{k}\left(S I L_{k}\right)
$$

\subsubsection{Dunn Index}

The distances between two clusters, $C_{j}$ and $C_{j^{\prime}}$, is measured by the distance between their closest points:

$$
d\left(C_{j}, C_{j^{\prime}}\right)=\min _{x_{i} \in C_{j}, x_{k} \in C_{j^{\prime}}}\left\|x_{i}-x_{k}\right\|
$$

and $d_{\min }$ is the minimum of these distances,

$$
d_{\text {min }}=\min _{C_{j} \neq C_{j^{\prime}}}\left(d\left(C_{j}, C_{j^{\prime}}\right)\right)
$$

For each cluster $C_{j}(j=1, \ldots, k)$, let $D_{j}$ be the distance between two elements of that cluster that are furthest apart. Note that this is sometimes denoted as the diameter of the cluster.

$$
D_{j}=\max _{x_{i}, x_{k} \in C_{j}, x_{i} \neq x_{k}}\left\|x_{j}^{i}-x_{j}^{k}\right\|
$$

and $d_{\max }$ is the largest of such distances,

$$
d_{\max }=\max _{j=1, . ., m}\left(D_{j}\right)
$$

In short, denoted by $d_{\min }$ is the smallest of the distance between two elements that belongs to different clusters and $d_{\max }$ as the maximum distance between two elements in 
one cluster. The Dunn index is defined as the ratio between $d_{\min }$ and $d_{\max }$,

$$
\operatorname{Dunn}_{k}=\frac{d_{\text {min }}}{d_{\max }}
$$

Underestimating the number of clusters will result into loose clusters where the samples are dispersed. This will result into a large value of $d_{\max }$ making the Dunn index small. On the other hand, when the number of cluster is overestimated, it will result into unnecessary splitting of one good compact clusters into two clusters that are very close to each other making $d_{\text {min }}$ small and the Dunn index small as well. The optimum number of clusters is therefore identified by finding the $k$ value that maximize the Dunn index.

$$
\hat{k}=\arg \max _{k}\left(\text { Dunn }_{k}\right)
$$

\subsubsection{Calinski-Harabasz index}

The Calinkski-Harabasz index is defined by the following:

$$
C H_{k}=\frac{N-k}{k-1} \frac{B G S S}{W G S S}
$$

where $W G S S$ was defined in 2.7 and the term $B G S S$ is known as between group dispersion. BGSS is a measure of how disperse the clusters that are formed. More specifically, BGSS is defined as the total distance of all the estimated cluster centers to the overall center of the whole data set. Letting $\mu_{x}$ be the center of the data set, BGSS is given by:

$$
B G S S=\sum_{j=1}^{k}\left(n_{j}\left\|c_{j}-\mu_{x}\right\|^{2}\right)
$$


where $n_{j}$ is the number of elements in cluster $C_{j}$ and $\mu_{x}$ is

$$
\mu_{x}=\frac{1}{N} \sum_{x_{i} \in x^{N}} x_{i}
$$

Typically, an ideal clustering solution provides a high BGSS which would happen if the clusters are well separated. It should also have a low WGSS which means that each cluster are compact. This being said, the optimum $k$ is estimated by maximizing $C H_{k}$

$$
\hat{k}=\arg \max _{k}\left(C H_{k}\right)
$$

\subsubsection{Davies-Bouldin Index}

The average distance of elements in cluster $C_{j}$ to its center $c_{j}$ is denoted by $\delta_{j}$.

$$
\delta_{j}=\frac{1}{n_{j}} \sum_{x_{i} \in C_{j}}\left\|x_{i}-c_{j}\right\|
$$

Denoted by $\left(C_{j}, C_{j^{\prime}}\right)$ is the distance between centers of cluster $C_{j}$ and $C_{j^{\prime}}$ where $j \neq j^{\prime}$.

$$
\left(C_{j}, C_{j^{\prime}}\right)=\left\|c_{j}-c_{j^{\prime}}\right\|
$$

Using (2.26) and (2.27), the Davies-Bouldin Index is given by the following:

$$
D B_{k}=\frac{1}{k} \sum_{j=1}^{k} \max _{j \neq j^{\prime}}\left(\frac{\delta_{j}+\delta_{j^{\prime}}}{\triangle_{j j^{\prime}}}\right)
$$

The quotient term $\left(\frac{\delta_{j}+\delta_{j^{\prime}}}{\triangle_{j j^{\prime}}}\right)$ can increase in two ways. The first one is by having sparse clusters which will effectively yield large values for $\delta_{j}$. Such case will happen when the number of clusters is underestimated. The second way that this quotient will increase 
is by making $\left(C_{j}, C_{j^{\prime}}\right)$ smaller, in other words, making two clusters close to each other, which is an effect of overestimation of number of clusters. This being said, the optimum $k$ is achieved by minimizing the Davies-Bouldin index.

$$
\hat{k}=\arg \min _{k}\left(D B_{k}\right)
$$

\subsubsection{Several Validity Index Behavior With Respect to $\mathrm{k}$}

In this part, we analyze the behavior of validity indexes discussed on the previous section when applied to data set with varying characteristics.

For Figure 2.1 and 2.2, where the data set is composed of IID clusters, optimization of the 5 validity indexes leads to a sensible estimate of the number of clusters.
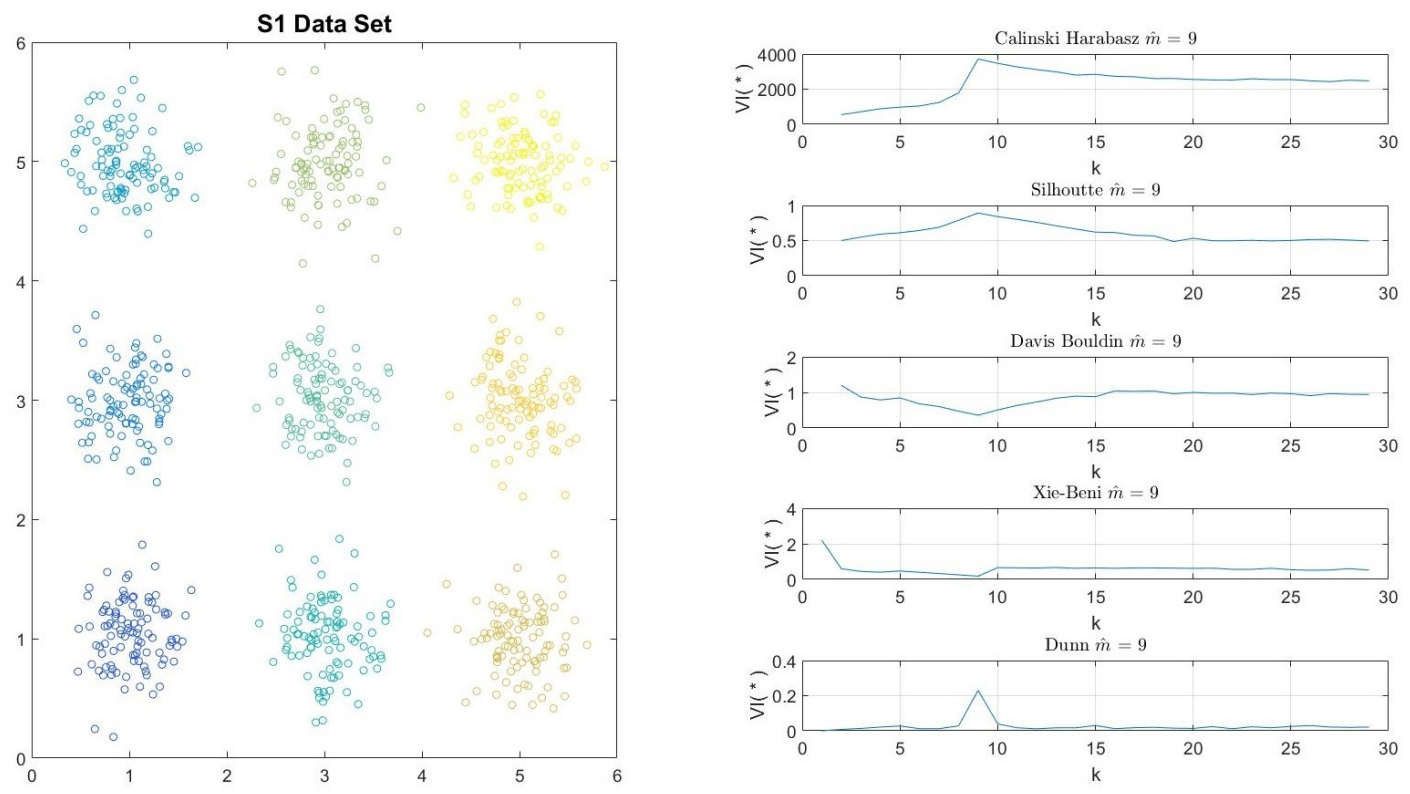

Figure 2.1: Validity index on data set with IID clusters, uniform variance and uniform proximity.

Figure 2.3 is the case when the clusters have a general Gaussian distribution and each cluster has a unique variance. The clusters in this cases are still distance-separated. 

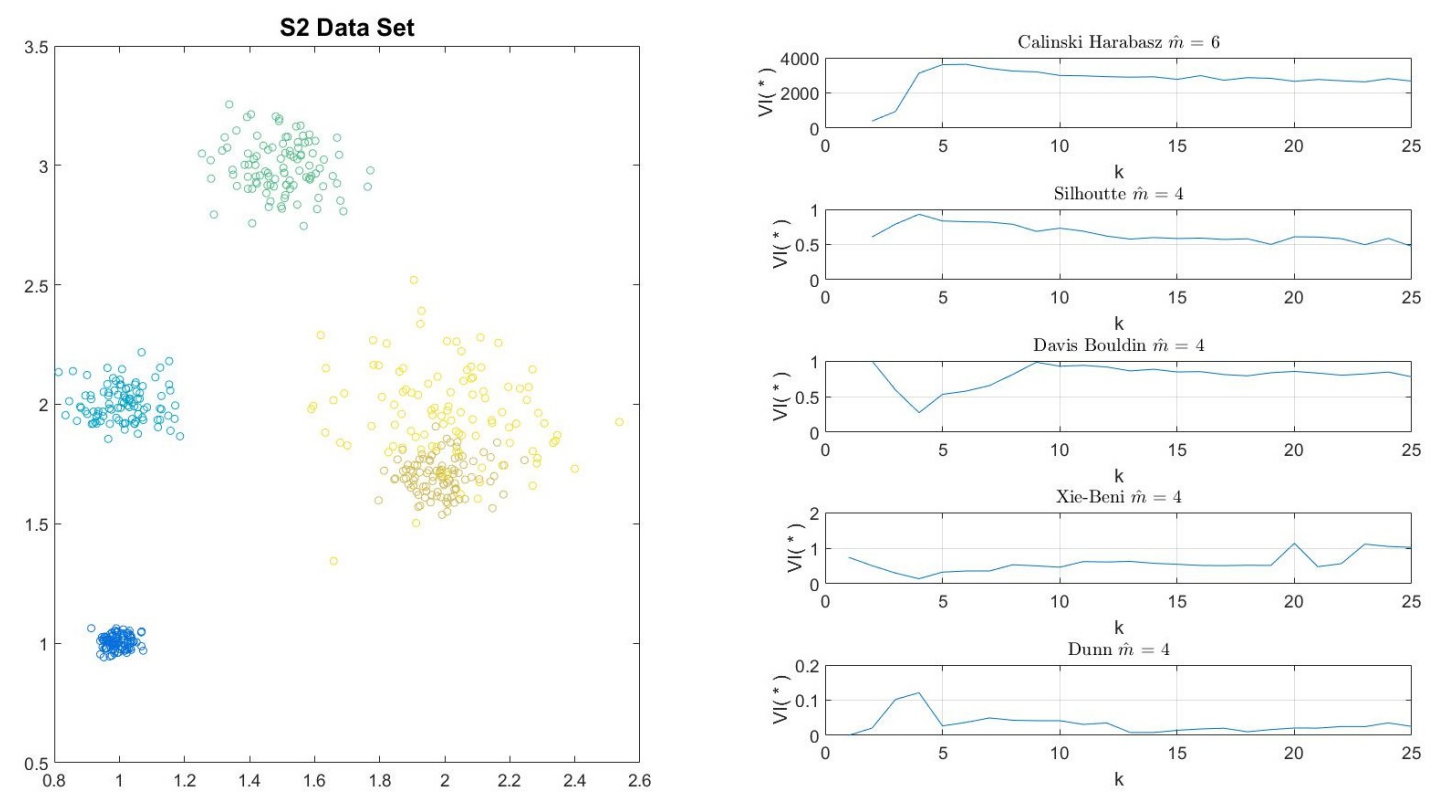

Figure 2.2: Validity index on data set with IID clusters, unique variance and inconsistent proximity.
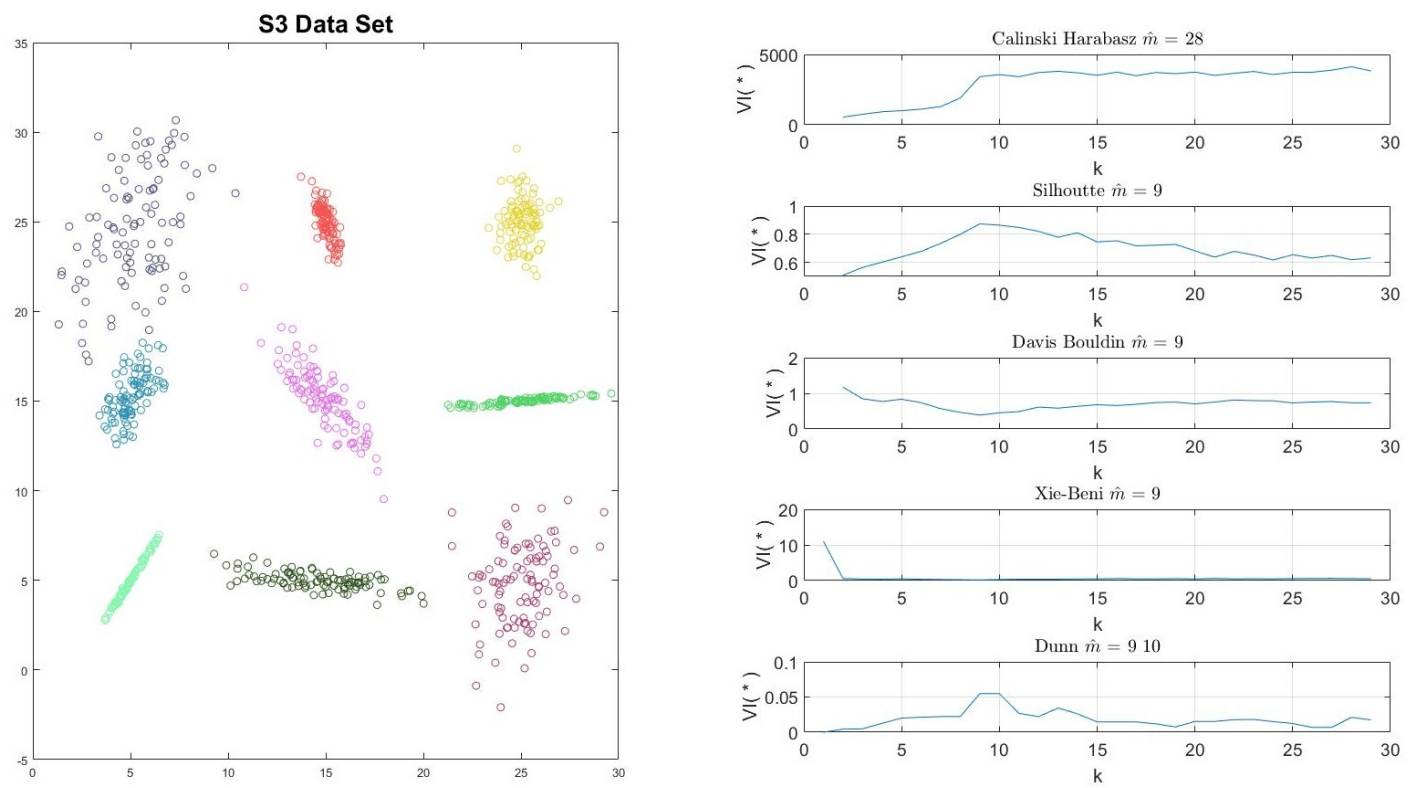

Figure 2.3: Validity index on data set with general Gaussian clusters, unique distribution and uniform proximity. 

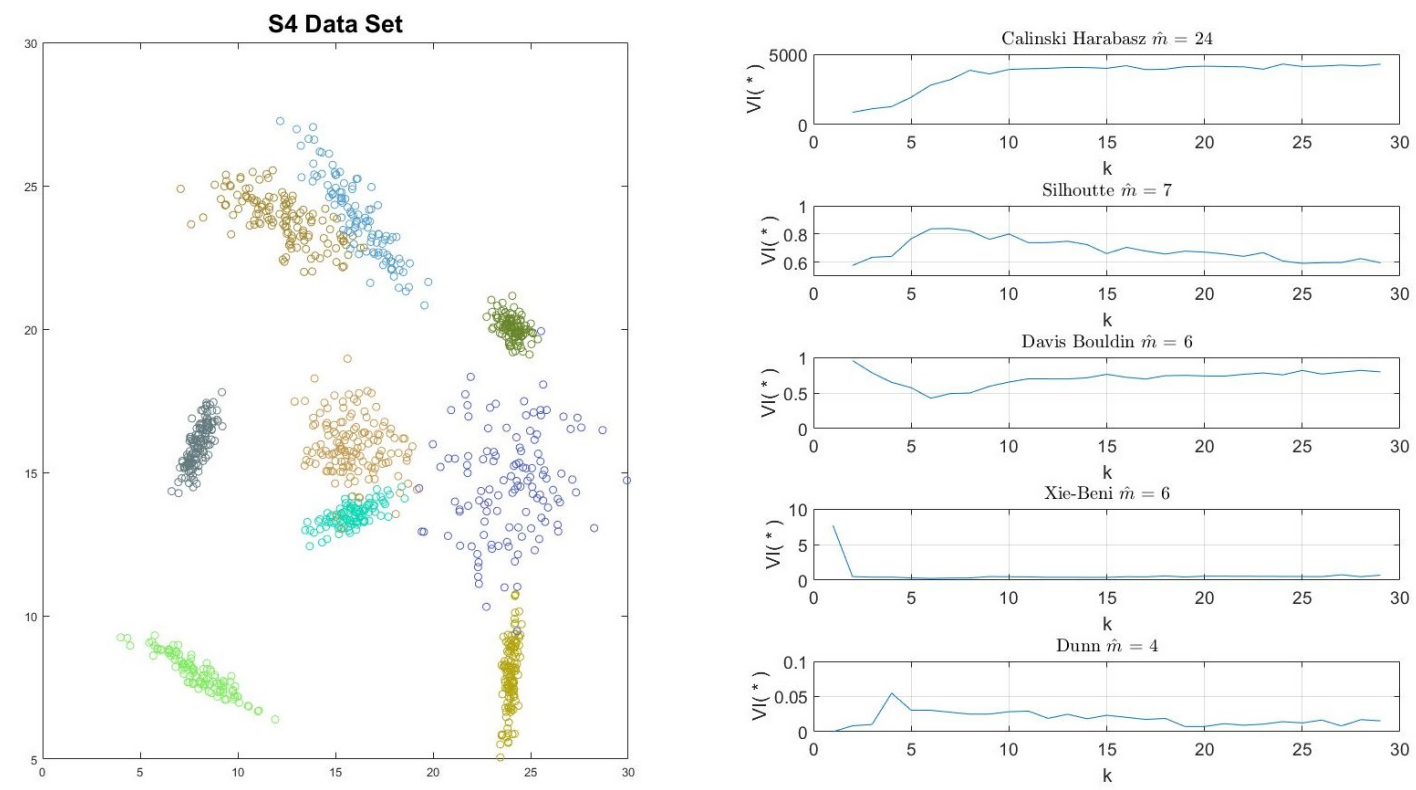

Figure 2.4: Validity index on data set with general Gaussian clusters, unique distribution and inconsistent proximity.
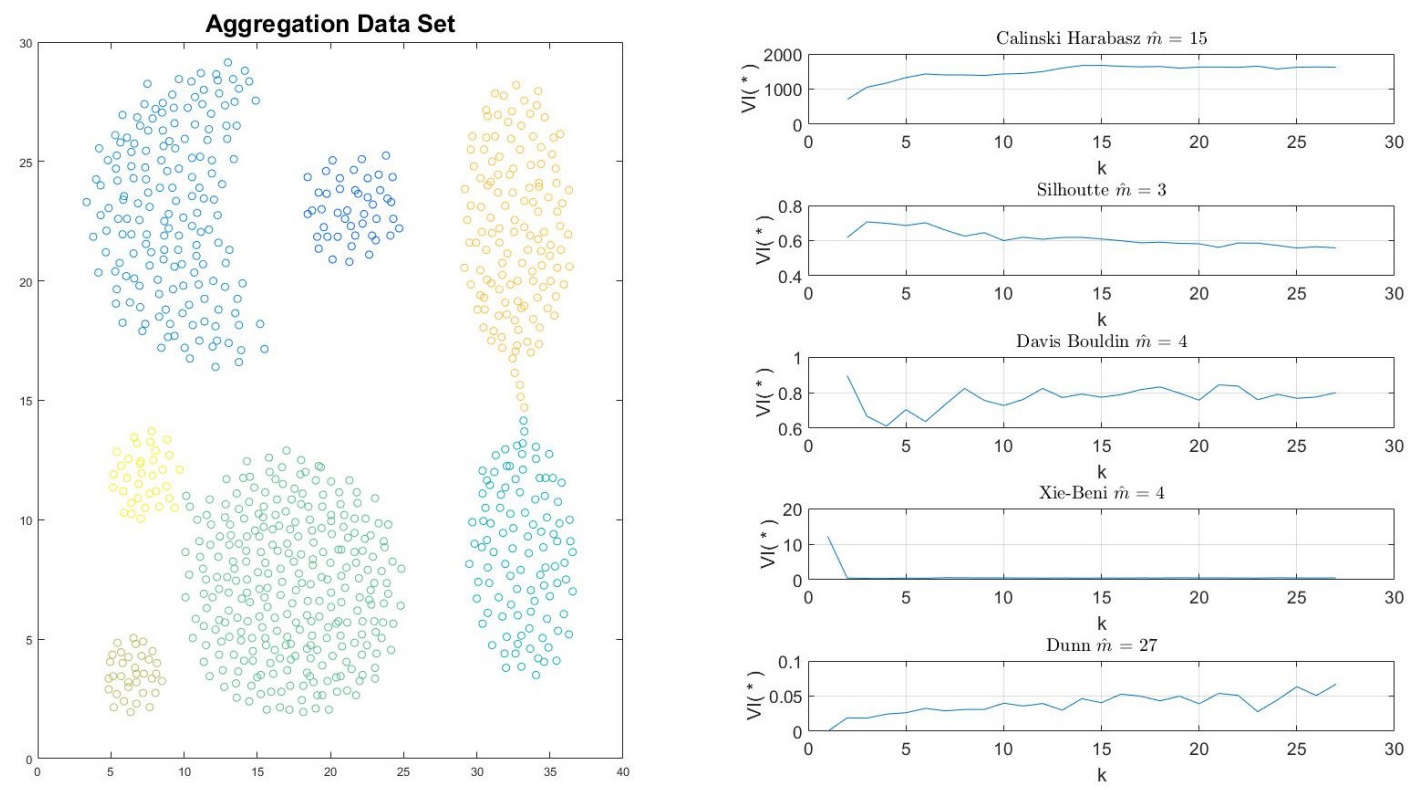

Figure 2.5: Validity index on Aggregation data set 29 

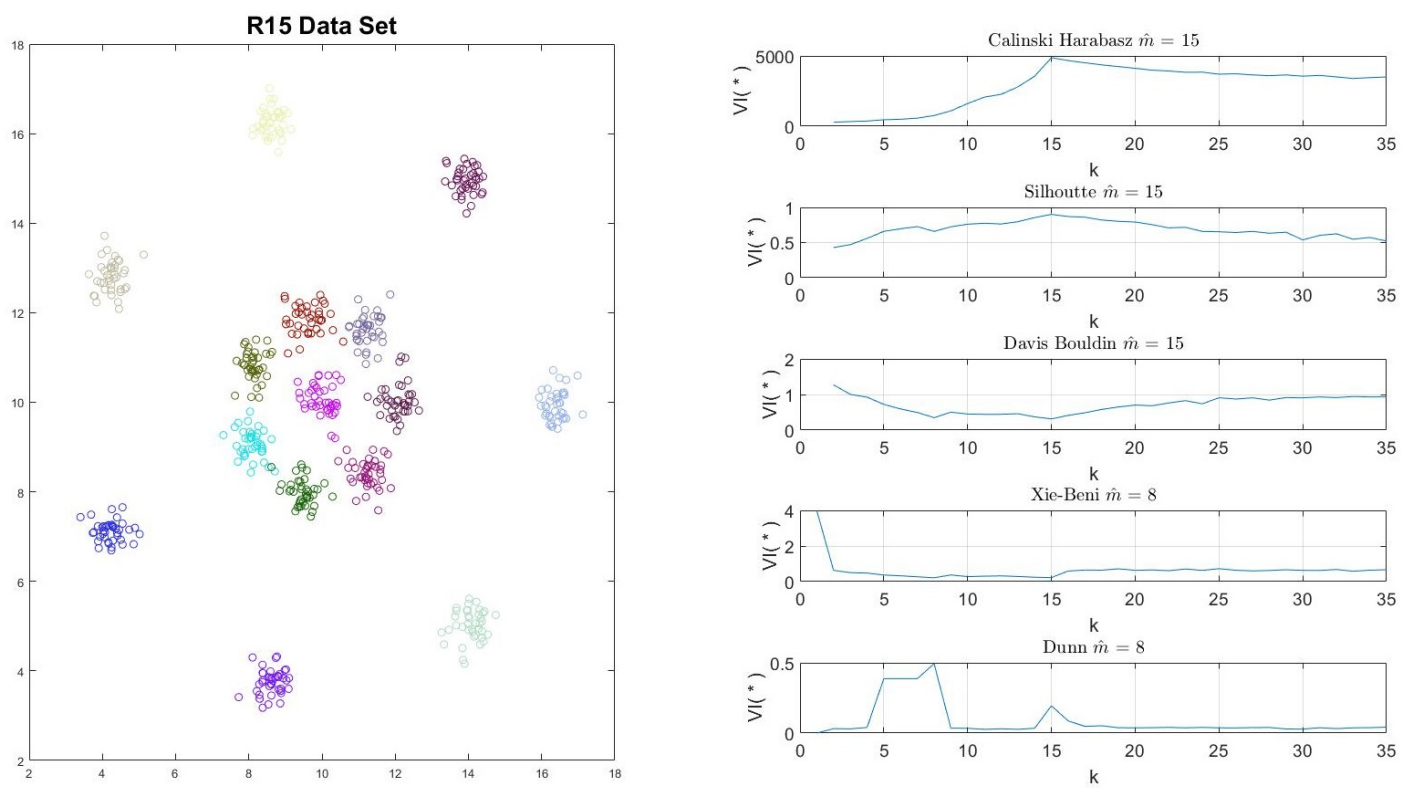

Figure 2.6: Validity index on R15 data set 30
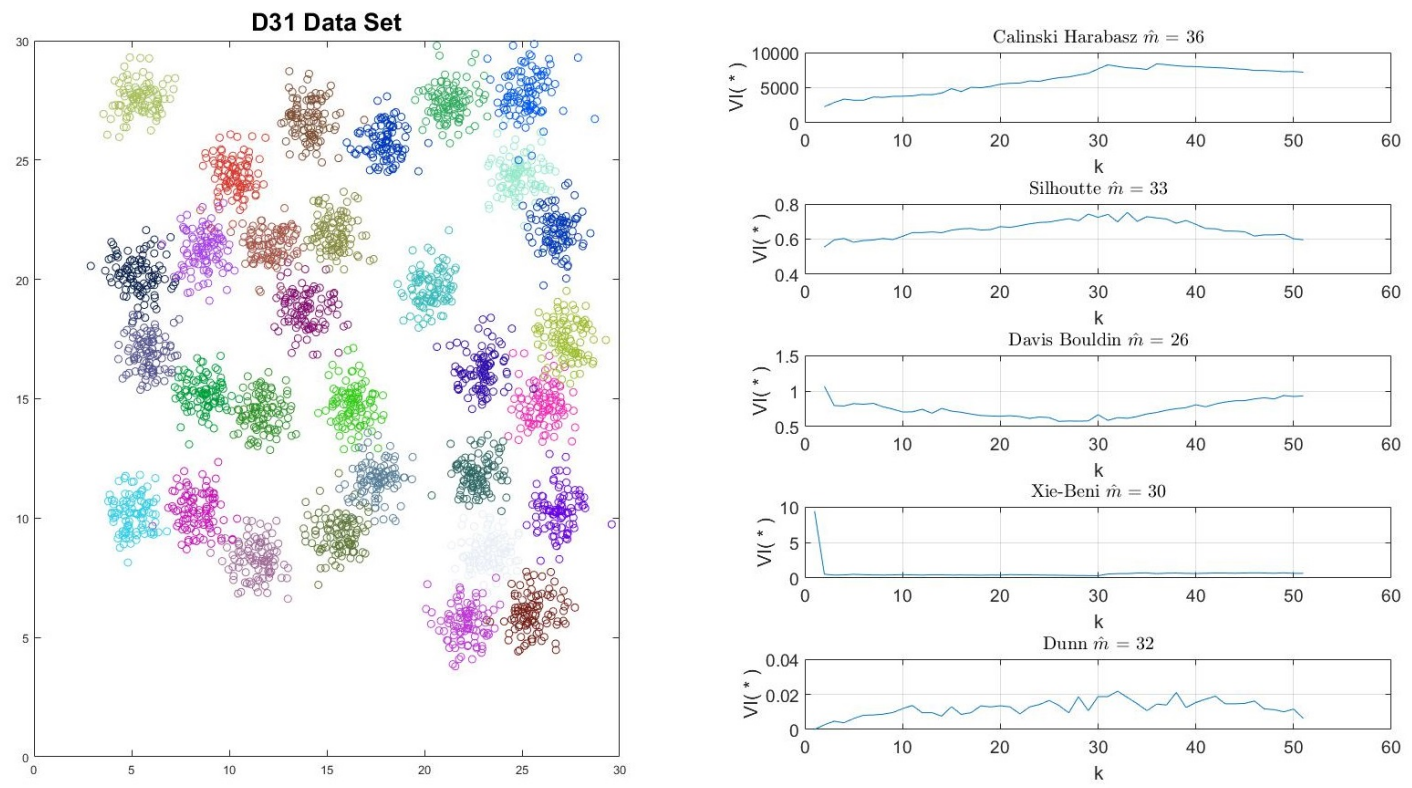

Figure 2.7: Validity index on D31 data set 30

The Calinkski-Harabasz index failed to give a proper estimate of the correct number of clusters but the other 4 validity indexes were able to. 
Figure 2.4 covers the case where each clusters has a unique Gaussian distribution and some of the clusters are overlapping. Aside from Calinski-Harabasz, the other 4 validity index method underestimated the number of clusters since it considered the overlapping clusters as 1 even though these overlapping clusters has a unique distribution. CalinskiHarabasz, is still unable to handle general Gaussian distributed clusters which explains why it inappropriately overestimated the number of clusters. From these result, it is evident that the 5 validity indeces method discussed works well for IID clusters but cannot handle General Gaussian clusters.

Figures 2.5-2.7 depicts data set introduced in 29] and 30. The 5 validity indexes method only provided sensible number of cluster estimate for data set S15 and D31 where all the clusters follows an IID distribution with the same variance. On the other hand, all 5 validity indexes performed poorly on the aggregation data set where all the clusters does not follow the same distribution and with some clusters connected through a thin neck.

\subsection{Information-Theoretic Approach to Signal De- noising and Best Basis Selection}

The observed noisy data $y^{N}=\left[y_{1}, \ldots, y_{N}\right]$ of length $N$ is available

$$
y[n]=\bar{y}[n]+w[n]
$$

where $\bar{y}^{N}$ is a unknown noiseless data of length $N$ and $w[n]$ is an additive white Gaussian noise that is a sample of the zero mean random variable $W(n)$ with variance $\sigma_{w}^{2}$

$$
w[n] \sim \mathcal{N}\left(0, \sigma_{w}^{2}\right)
$$


Expressing the observed data $y^{N}$ as well as the noiseless data $\bar{y}^{N}$ in terms of a desired orthonormal basis, we have:

$$
\theta[i]=<s_{i}, y^{N}>\quad, \quad \bar{\theta}[i]=<s_{i}, \bar{y}^{N}>\quad, \quad i=1,2, \ldots, N
$$

where $\langle *, *\rangle$ is the inner product between two vectors and the orthonormal basis vectors $s^{N}=\left[s_{1}, s_{2}, \ldots, s_{N}\right]$ has the following properties.

$$
<s_{i}, s_{j}>= \begin{cases}1 & i=j \\ 0 & i \neq j\end{cases}
$$

The observed data as well as the unknown noiseless data can be reconstructed using the following:

$$
y^{N}=\sum_{i=1}^{N} \theta[i] s_{i} \quad, \quad \bar{y}^{N}=\sum_{i=1}^{N} \bar{\theta}[i] s_{i}
$$

Due to orthonormality of the basis vectors, the noise coefficients $v[i] \bigsqcup^{2}$ are also IID random variables with zero mean and variance of $\sigma_{w}^{2}$. The relationship between these 3 coefficients are as follows:

$$
\theta[i]=\bar{\theta}[i]+v[i]
$$

Under the assumption that the noiseless signal is recoverable from the observed noisy signal, it is implied that the noise variance is small meaning noise coefficients $v[i]$ are tightly distributed around its mean which is zero. As a result, noise coefficients $v[i]$, will tend to have small values as compared to the noiseless signal coefficients $\bar{\theta}[i]$.

Consider $s^{m}$ which is a subspace of $s^{N}$ that is spanned by $m$ elements $(m<N)$. An ${ }^{2} v[i]=<s_{i}, w^{N}>, \quad i=1,2, \ldots, N$ 
estimate of the noiseless data in this subspace can be given by

$$
\hat{y}_{s_{m}}^{N}=\sum_{i=1}^{m} \hat{\theta}_{s_{m}}[i] s_{i}
$$

and the estimated coefficient $\hat{\theta}_{s_{m}}[i]$ is derived from $\theta[i]$ such that

$$
\hat{\theta}_{S_{m}}[i]= \begin{cases}\theta[i] & \text { if } s_{i} \in s^{m} \\ 0 & \text { otherwise }\end{cases}
$$

Denoising is achieved by selecting subspace $s^{m} \in s^{N}$. In other words, we project the observed noisy signal in subspace $s^{m}$ with the hopes that such projection will result into elimination of noise coefficients. The objective is then to find this subspace $s^{m}$. To approach this problem, the following two error measurement is defined:

$$
\begin{gathered}
\text { Data Error : } y_{s_{m}}=\frac{1}{N}\left\|y^{N}-\hat{y}_{s_{m}}^{N}\right\|_{2}^{2} \\
\text { Reconstruction Error : } z_{s_{m}}=\frac{1}{N}\left\|\bar{y}^{N}-\hat{y}_{s_{m}}^{N}\right\|_{2}^{2}
\end{gathered}
$$

The difference between the observed noisy signal $y^{N}$ and the estimated signal $\hat{y}_{s_{m}}$, which is observed signal's projection to subspace $s^{m}$, is referred to as the the data error $y_{s_{m}}$. The Reconstruction error $z_{s_{m}}$ on the other hand, is the difference between between the estimated signal and the noiseless signal. The minimization of $z_{s_{m}}$ error leads to an optimum solution of subspace selection. In real life however, the noiseless signal is unavailable making $z_{s_{m}}$ an unobservable error. Through probabilistic validation, this unobservable error can be estimated using the available data error. 


\subsubsection{Estimation of Reconstruction Error from Data Error}

The data error $y_{s_{m}}$ is a sample of random variable $Y_{s_{m}}$. Expected value and variance of the data error can be derived (Refer to 22 for more details).

$$
\begin{gathered}
E\left[Y_{s_{m}}\right]=\frac{N-m}{N} \sigma_{w}^{2}+\frac{1}{N}\left\|\triangle_{S_{m}}\right\|_{2}^{2} \\
\operatorname{Var}\left[Y_{s_{m}}\right]=\frac{2}{N}\left(\frac{N-m}{N}\right)\left(\sigma_{w}^{2}\right)^{2}+\frac{4 \sigma_{w}^{2}}{N^{2}}\left\|\triangle_{S_{m}}\right\|_{2}^{2}
\end{gathered}
$$

where the term $\left\|\triangle_{s_{m}}\right\|_{2}^{2}$ is a component that is due to the noiseless signal. The calculation of this term in unavailable since information about the noiseless signal is required. Refer to 22 for more details.

Although $Y_{s_{m}}$ follows a Chi-square distribution, central limit theorem can be applied which means that with a large value for $N$, both $Y_{s_{m}}$ and $Z_{s_{m}}$ can be estimated by a Gaussian distribtuion. This allows for derivation of mathematical expressions for the

bounds on the $y_{s_{m}}$ and $z_{s_{m}}$. Through probabilistic validation, the term $\left\|\triangle_{S_{m}}\right\|_{2}^{2}$ is estimated using the observed data error $y_{s_{m}}$. The probabilistic validation assumes that the observed $y_{s_{m}}$ is always worst case of $Y_{s_{m}}$ meaning the following:

$$
E\left[Y_{s_{m}}\right]-\alpha \sqrt{\operatorname{Var}\left[Y_{s_{m}}\right]}=y_{S_{m}}
$$

The parameter $\alpha$ is chosen based on validation probability $P_{2}$ such that $y_{s_{m}}$ is always within $\alpha \sqrt{\operatorname{Var}\left[Y_{S_{m}}\right]}$ away from its mean with a probability $P_{2}$.

$$
P\left(\left|E\left[Y_{S_{m}}\right]-y_{S_{m}}\right| \leq \alpha \sqrt{\operatorname{Var}\left[Y_{S_{m}}\right]}\right)=P_{2}
$$

Plugging in (2.38) and $(2.39)$ in $(2.40)$ and by choosing an appropriate $\alpha$ so that 
$P_{2} \approx 1$, an estimate of the bounds of the unknown term $\left\|\triangle_{S_{m}}\right\|_{2}^{2}$ is obtained from the observed $y_{s_{m}}$.

$$
\begin{aligned}
& \frac{1}{N} \overline{\left\|\triangle_{S_{m}}\right\|_{2}^{2}}=y_{s_{m}}-\frac{(N-m) \sigma_{w}^{2}}{N}+\frac{2 \alpha^{2} \sigma_{w}^{2}}{N}+K_{s_{m}}(\alpha) \\
& \frac{1}{N} \frac{\left\|\triangle_{S_{m}}\right\|_{2}^{2}}{N}=y_{s_{m}}-\frac{(N-m) \sigma_{w}^{2}}{N}+\frac{2 \alpha^{2} \sigma_{w}^{2}}{N}-K_{s_{m}}(\alpha)
\end{aligned}
$$

and

$$
K_{s_{m}}(\alpha)=2 \alpha \frac{\sigma_{w}}{\sqrt{N}} \sqrt{\frac{\alpha^{2} \sigma_{w}^{2}}{N}+y_{s_{m}}-\frac{(N-m) \sigma_{w}^{2}}{2 N}}
$$

Similarly, the reconstruction error $z_{s_{m}}$ is a sample of the random variable $Z_{s_{m}}$ whose mean and variance are as follows:

$$
\begin{gathered}
E\left[Z_{s_{m}}\right]=\frac{m}{N} \sigma_{w}^{2}+\frac{1}{N}\left\|\triangle_{S_{m}}\right\|_{2}^{2} \\
\operatorname{Var}\left[Z_{s_{m}}\right]=\frac{2 m}{N^{2}}\left(\sigma_{w}^{2}\right)^{2}
\end{gathered}
$$

The first term of $E\left[Z_{s_{m}}\right]$ is due to the noise component and is an increasing function of $m$. The second term on the other hand is a decreasing function of $m$. This relationship is traditionally called the bias-variance trade off.

The actual reconstruction error $z_{s_{m}}$ is within $\beta \sqrt{\operatorname{Var}\left[Z_{S_{m}}\right]}$ away from its mean with a desired probability $P_{1}$.

$$
P\left(\left|E\left[Z_{S_{m}}\right]-z_{S_{m}}\right| \leq \beta \sqrt{\operatorname{Var}\left[Z_{S_{m}}\right]}\right)=P_{1}
$$

where $P_{1}$ is referred as the confidence probability and it is desired that $P_{1} \approx 1$. To 
cater to this requirement, the parameter $\beta$ is controlled.

The actual reconstruction error $z_{s_{m}}$ is therefore bounded such that

$$
\begin{gathered}
\underline{Z_{S_{m}}} \leq z_{s_{m}} \leq \overline{Z_{S_{m}}} \\
\overline{Z_{S_{m}}}=E\left[Z_{S_{m}}\right]+\beta \sqrt{\operatorname{Var}\left[Z_{S_{m}}\right]} \\
\underline{Z_{S_{m}}}=E\left[Z_{S_{m}}\right]-\beta \sqrt{\operatorname{Var}\left[Z_{S_{m}}\right]}
\end{gathered}
$$

The term $\left\|\triangle_{S_{m}}\right\|_{2}^{2}$ is needed to calculate for the expected value of reconstruction error from 2.44. But by using the estimated bounds of $\left\|\triangle_{S_{m}}\right\|_{2}^{2}$ derived from 2.42, the bounds of $z_{s_{m}}$ is estimated using only the available data error $y_{s_{m}}$.

$$
\begin{aligned}
& \overline{Z_{S_{m}}}=\frac{m}{N} \sigma_{w}^{2}+\frac{1}{N} \overline{\left\|\triangle_{S_{m}}\right\|_{2}^{2}}+\beta \frac{\sigma_{2}^{2} \sqrt{2 m}}{N} \\
& \underline{Z_{S_{m}}}=\frac{m}{N} \sigma_{w}^{2}+\frac{1}{N} \frac{\left\|\triangle_{S_{m}}\right\|_{2}^{2}}{N}-\beta \frac{\sigma_{2}^{2} \sqrt{2 m}}{N}
\end{aligned}
$$

Using (2.49), the unobservable error $Z_{s_{m}}$ can be estimated on any subspace $s^{m}$. 



\section{Chapter 3}

\section{k-MACE Clustering}

Clustering methods that attempts to estimate the correct number of clusters have to deal with two optimization problems simultaneously. One of which is estimating the optimum number of cluster and the other one is finding the optimum partition based on the estimated number of clusters. Algorithms such as k-means and Fuzzy-C means deals with the latter but requires the number of clusters as an input. We define the average central error as the difference between the true cluster center and the estimated cluster center. Minimization of this error allows for simultaneous optimization of the partitioning problem as well as the estimation of number of clusters. However, to be able to solve for this error, one would need the true cluster center which is unavailable. Inspired by [21, we provide a probabilistic approach to estimate this unobservable error and use such estimate to find the optimum number of clusters.

This chapter is organized as follows: Section 3.1 concentrates on preliminaries and notations that were used. Section 3.2 concentrates on our propose clustering method denoted by $\mathrm{k}-\mathrm{MACE}$. Section 3.3 presents our method for estimating covariances that are required in k-MACE clustering. Finally, Section 3.4 analyzes k-MACE compu- 
tational complexity.

\subsection{Preliminaries and Notations}

\subsubsection{Data Model}

Tables 3.1 and 3.2 provides a list of symbols that were used for formulations of $\mathrm{k}-\mathrm{MACE}$ and $\mathrm{k}-\mathrm{MACE}_{\sigma}$. Consider an observed data of length $N, x^{N}=\left[x_{1}, x_{2}, \ldots, x_{N}\right]^{T}$ where $x_{i} \in R_{1 \times d}$. Each element $x_{i}$ is a vector of length $d$, and each scalar element of $x_{i}$ represents an observed feature. The observed data $x_{i}$ is a sample of random vector $X_{i}$, with the following statement:

$$
X_{i}=\bar{c}_{X_{i}}+\bar{W}_{X_{i}}
$$

$\bar{W}_{X_{i}}$ is a random vector that describes the variation of element $X_{i}$ around its center $\bar{c}_{X_{i}}$. We assume that this variation follows a zero mean Gaussian distribution, therefore:

$$
X_{i}=\bar{c}_{X_{i}}+\mathcal{N}\left(0, \bar{\Sigma}_{X_{i}}\right)
$$

where $\bar{\Sigma}_{X_{i}}$ is a $d \times d$ covariance matrix.

The data set is generated by $\bar{m}$ cluster model such that

$$
X^{N}=\bar{C}_{1} \cup \bar{C}_{2} \cup \ldots \cup \bar{C}_{\bar{m}}
$$

and

$$
\bar{C}_{i} \cap \bar{C}_{j}=\emptyset, \quad i \neq j
$$


Table 3.1: Table of symbols used in $\mathrm{k}-\mathrm{MACE}$ and $\mathrm{k}-\mathrm{MACE}_{\sigma}$ formulations Part 1

\begin{tabular}{|c|c|}
\hline Symbol & Description \\
\hline$x^{N}$ & Data set to be clustered \\
\hline$N$ & Length of Data Set \\
\hline$d$ & Dimension of data / Number of Features \\
\hline$x_{i}$ & The $i^{t h}$ element of data Set $x^{N}$. Sampled from Random vector $X_{i}$ \\
\hline $\bar{m}$ & Correct number of cluster \\
\hline $\bar{c}_{X_{i}}$ & Center of Random Variable $X_{i}$ \\
\hline $\bar{W}_{X_{i}}$ & $\begin{array}{l}\text { A dependent random vector that is describes variation of } X_{i} \text { around its center. } \\
\bar{W}_{X_{i}} \text { is z zero mean Gaussian Distribution }\end{array}$ \\
\hline $\bar{\Sigma}_{X_{i}}$ & Covariance of Random Vector $\bar{W}_{X_{i}}$ \\
\hline $\bar{\Lambda}_{X_{i}}$ & Diagonal matrix whose elements are the eigenvalues of $\bar{\Sigma}_{X_{i}}$ \\
\hline $\bar{C}_{j}$ & $j^{\text {th }}$ cluster of the true clustering partition \\
\hline $\bar{c}_{j}$ & Center of cluster $\bar{C}_{j}$ \\
\hline $\bar{\Sigma}_{j}$ & Covariance of true cluster $\bar{C}_{j}$ \\
\hline $\bar{\Lambda}_{j}$ & Diagonal matrix whose elements are the eigenvalues of $\bar{\Sigma}_{j}$ \\
\hline $\bar{Q}_{j}$ & Modal matrix of $\bar{\Sigma}_{j}$ \\
\hline$m$ & Number of clusters \\
\hline$C_{m j}$ & $j^{\text {th }}$ cluster in $m$-clustering step \\
\hline$\hat{c}_{m j}$ & Center of cluster $C_{m j}$ \\
\hline
\end{tabular}


Table 3.2: Table of symbols used in $\mathrm{k}-\mathrm{MACE}$ and $\mathrm{k}-\mathrm{MACE}_{\sigma}$ formulations Part 2

\begin{tabular}{|c|c|}
\hline Symbol & Description \\
\hline$n_{m j}$ & Number of Elements in cluster $C_{m j}$ \\
\hline$x_{m j}$ & All the elements of cluster $C_{m j}$ \\
\hline$x_{m j}^{i}$ & $i^{t h}$ element of cluster $C_{m j}$ \\
\hline$Z_{s_{m j}}$ & Central error of cluster $C_{m j}$ \\
\hline$Y_{s_{m j}}$ & Data error of cluster $C_{m j}$ \\
\hline $\bar{c}_{x_{m j}}$ & True center of all the elements in cluster $C_{m j}$ \\
\hline$c_{m j}$ & Estimated center of all the elements in cluster $C_{m j}$ \\
\hline$Z_{s_{m}}$ & Average central error of $m$-clustering step \\
\hline$\overline{Z_{s_{m}}}, \underline{Z_{s_{m}}}$ & Upper bound and lower bound of $Z_{s_{m}}$, respectively \\
\hline$Y_{s_{m}}$ & Average data error of $m$-clustering step \\
\hline$\left\|A_{m j} \bar{c}_{m j}\right\|_{F}^{2}$ & Unknown term that is necessary for calculation of ACE. \\
\hline$\overline{\left\|A_{m j} \bar{c}_{m j}\right\|_{F}^{2}},\left\|A_{m j} \bar{c}_{m j}\right\|_{F}^{2}$ & Upper bound and lower bound of $\left\|A_{m j} \bar{c}_{m j}\right\|_{F}^{2}$, respectively \\
\hline$\hat{W}_{x_{m j}^{i}}$ & $\begin{array}{l}\text { Independent random vector that describes variation of } \\
x_{m j}^{i} \text { around its true center }\end{array}$ \\
\hline $\bar{\Lambda}_{x_{m j}^{i}}$ & Covariance matrix of $\hat{W}_{x_{m j}^{i}}$ \\
\hline $\bar{\sigma}_{w}^{2}$ & Variance of uniform IID clusters. \\
\hline$\hat{\sigma}_{w}^{2}$ & An estimate of $\bar{\sigma}_{w}^{2}$. \\
\hline$\hat{m}$ & Estimated number of clusters. \\
\hline
\end{tabular}


Consider cluster $\bar{C}_{j} \in\left[\bar{C}_{1}, \bar{C}_{2}, \ldots, \bar{C}_{\bar{m}}\right]$, we can write down its elements as follows:

$$
\left[\begin{array}{c}
X_{1} \\
\vdots \\
X_{n_{j}}
\end{array}\right]=\left[\begin{array}{c}
\bar{c}_{j} \\
\vdots \\
\bar{c}_{j}
\end{array}\right]+\left[\begin{array}{c}
\mathcal{N}\left(0, \bar{\Sigma}_{j}\right) \\
\vdots \\
\mathcal{N}\left(0, \bar{\Sigma}_{j}\right)
\end{array}\right]
$$

where $n_{j}$ is the number of elements in cluster $\bar{C}_{j}, \bar{c}_{j}$ and $\bar{\Sigma}_{j}$ are the center and the covariance of cluster $\bar{C}_{j}$, respectively.

Note that the covariance matrix $\bar{\Sigma}_{j}$ is a symmetric matrix and $\bar{\Sigma}_{j}=\bar{Q}_{j} \bar{\wedge}_{j} \bar{Q}_{j}^{T}$ where $\bar{\Lambda}_{j}$ is a diagonal matrix whose elements are the eigenvalues of $\bar{\Sigma}_{j} \cdot \bar{Q}_{j}$ is an orthonormal matrix that is the eigenvector of $\bar{\Sigma}_{j} . \bar{W}_{j}$, can therefore be simplified in terms of an independent Gaussian random vector $\hat{W}_{j}$ such that $\bar{W}_{j}=\hat{W}_{j} \bar{Q}_{j}$ where $\hat{W}_{j} \sim \mathcal{N}\left(0, \bar{\Lambda}_{j}\right)$. Rewriting (3.5) in terms of diagonal matrix $\bar{\wedge}_{j}$, we have

$$
\left[\begin{array}{c}
X_{1} \\
\vdots \\
X_{n_{j}}
\end{array}\right]=\left[\begin{array}{c}
\bar{c}_{j} \\
\vdots \\
\bar{c}_{j}
\end{array}\right]+\left[\begin{array}{c}
\mathcal{N}\left(0, \bar{\wedge}_{j}\right) \bar{Q}_{j} \\
\vdots \\
\mathcal{N}\left(0, \bar{\wedge}_{j}\right) \bar{Q}_{j}
\end{array}\right]
$$

From (3.3) and (3.4), it is stated that all elements of the data set $X^{N}$ should come from either one of the $\bar{m}$ clusters. It should follow that:

$$
\bar{c}_{X_{i}} \in\left[\bar{c}_{1}, \ldots, \bar{c}_{\bar{m}}\right]^{T}, \bar{\Sigma}_{X_{i}} \in\left[\bar{\Sigma}_{1}, \ldots, \bar{\Sigma}_{\bar{m}}\right]^{T}, \bar{\wedge}_{X_{i}} \in\left[\bar{\wedge}_{1}, \ldots, \bar{\wedge}_{\bar{m}}\right]^{T} \forall X_{i} \in X^{N}
$$




\subsubsection{Clustering the Available Data Into $m$ Clusters}

Clustering the available data into $m$ clusters results in $m$ cluster centers. Each of these $m$ cluster center is denoted by $\hat{c}_{m j}$. The $j^{\text {th }}$ cluster is denoted by $C_{m j},(j=1, \ldots, m)$. 1 Clustering the data using k-means, the cluster center is calculated by averaging all the elements that belongs to that cluster such that:

$$
\hat{c}_{m j}=\frac{1}{n_{m j}} \sum_{x_{i} \in C_{m j}} x_{i}
$$

where $n_{m j}$ is the number of elements in $C_{m j}$. Note that $\hat{c}_{m j}$ is a vector of length $d$ (same dimension as $x_{i}$ ). In our formulation, we denote the elements in cluster $C_{m j}$ by the term $x_{m j}$ and the $i^{t h}$ element of cluster $C_{m j}$ is denoted by $x_{m j}^{i}$. Consequently, the data set $x^{N}$, when split into $m$ clusters, can be expressed as follows:

$$
\begin{array}{r}
x^{N}=x_{m 1} \cup x_{m 2} \cup \ldots \cup x_{m m} \\
x_{m i} \cap x_{m j}=\emptyset, \quad i \neq j
\end{array}
$$

Figure 3.7 depicts an example that shows variables $\bar{C}_{j}, \bar{c}_{j}, C_{m j}$ and $\hat{c}_{m j}$

\subsubsection{Average Central Error}

Estimating the CNC is approached by comparing clustering result when the data set is split into $m$ clusters for a given range of $m\left(\left[m_{\min }: m_{\max }\right]\right)$. The goal is to have the estimated $\mathrm{CNC}, \hat{m}$ as close as possible to the true number of clusters $\bar{m}$. For this comparison, we proposed to use the ACE for each $m$ clustering and the $m$ that minimizes

\footnotetext{
${ }^{1}$ the subscript $m j$ pertains to the $j^{\text {th }}$ cluster of the $m$-clustering result
} 


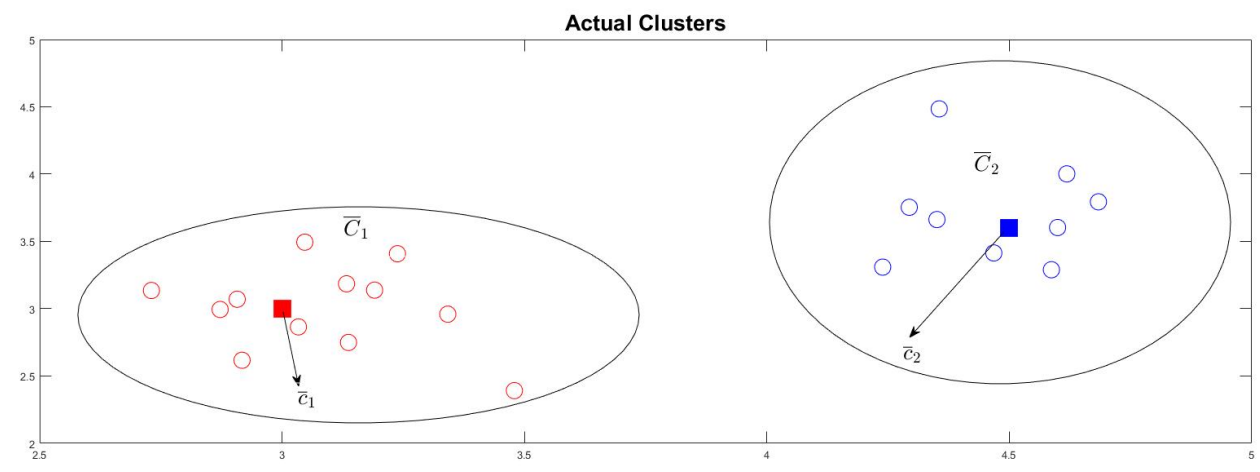

(a) Actual Clusters

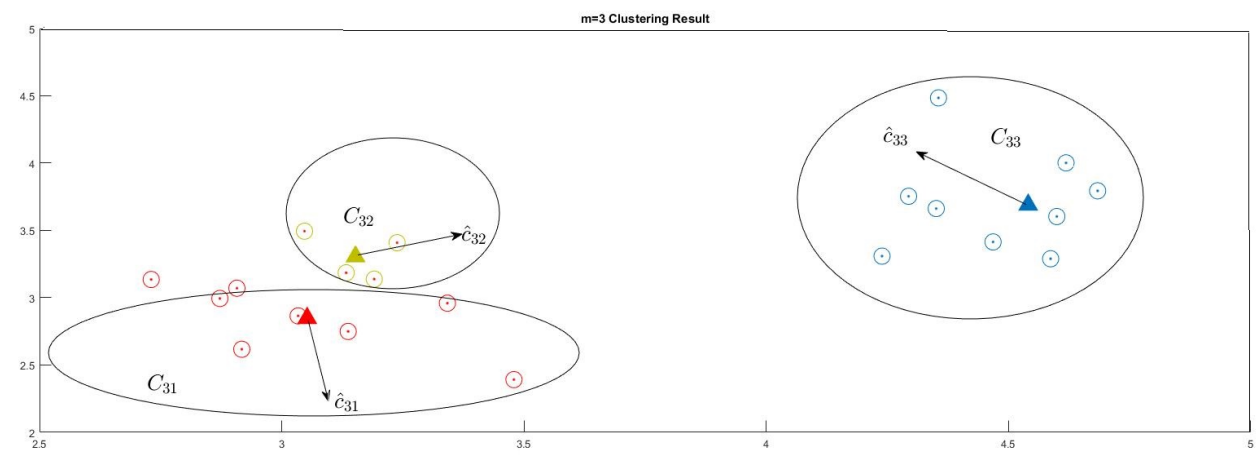

(b) $\mathrm{m}=3$ Clustering Step

Figure 3.1: Example of clustering solution and its corresponding estimated centers $\left(\hat{c}_{m j}\right)$ as well as the true cluster centers $\left(\bar{c}_{j}\right)$

ACE is the estimated CNC. 2

The average central error is the error measurement that we desire to use in evaluating a clustering solution. k-Minimizing Average Central Error (k-MACE) as the name suggest aims to look for $k$ (number of cluster input in k-means) in which the average central error is minimized. The ACE, denoted by $Z_{s_{m}}$, is described as the average distance between the estimated cluster center and the true cluster center. ACE is a function of $m$ and is

\footnotetext{
${ }^{2} \mathrm{k}-\mathrm{MACE}$ formulation of the ACE is a variation of what was defined in 24 where $Z_{s_{m j}}=$ $\frac{1}{n_{m j}}\left\|\bar{c}_{x_{m j}}-c_{m j}\right\|_{F}^{2}$ and $Z_{s_{m}}=\frac{1}{m} \sum_{j=1}^{m} Z_{s_{m j}}$
} 
formulated as follows:

$$
Z_{s_{m}}=\frac{1}{N} \sum_{j=1}^{m} Z_{s_{m j}}
$$

and

$$
Z_{s_{m j}}=\left\|\bar{c}_{x_{m j}}-c_{m j}\right\|_{F}^{2}
$$

where $\bar{c}_{x_{m j}}$ is an $\left(n_{m j} \times d\right)$ matrix that will be formed by taking the true cluster center associated with all the elements in cluster $x_{m j}$.

$$
\bar{c}_{x_{m j}}=\left[\bar{c}_{x_{1}}, \ldots, \bar{c}_{x_{n_{m j}}}\right]^{T} \quad \mid x_{i} \in x_{m j}
$$

The term $c_{m j}=\overrightarrow{1}_{n_{m j}} \hat{c}_{m j}$, and $\overrightarrow{1}_{n_{m j}}$ is a column vector of ones with length $n_{m j}$, $\hat{c}_{m j}$ is from (3.8) and the term $\|(*)\|_{F}$ refers to the Frobenius norm.

The calculation of the Average central error requires the knowledge of the true cluster centers. For this reason, the ACE is said to be unobservable. On the other hand, the data error, as will be introduced in the next section, is observable for all $m$. In Section 3.2, we propose a method for estimating ACE by using the available data error that is defined in the following section.

\subsubsection{Data Error}

The Data error denoted by $\left.Y_{s_{m}}\right]^{3}$ can also be referred as the cluster compactness. It is a measure of how far the samples of a cluster are to the cluster's estimated center. Data error, commonly known as WGSS are also used in other index validation techniques such as Xie-Beni and intra-cluster correlation coefficient 24, as it provides a measure of

\footnotetext{
${ }^{3} \mathrm{k}-\mathrm{MACE}$ definition of data compactness is a variation of what was defined in 23 where $Y_{s_{m j}}=$ $\frac{1}{n_{m j}}\left\|x_{m j}-c_{m j}\right\|_{F}^{2}$ and $Y_{s_{m}}=\frac{1}{m} \sum_{j=1}^{m} Y_{s_{m j}}$
} 
cluster cohesion, that is, how closely the objects are related in one cluster. The data error is a decreasing function of $m$ and on the extreme case where $m=N$ (i.e each sample is its own cluster) $Y_{s_{m}}$ is equal to $0 . Y_{s_{m}}$ is given by the following formula:

$$
Y_{s_{m}}=\frac{1}{N} \sum_{j=1}^{m} Y_{s_{m j}}
$$

and

$$
Y_{s_{m j}}=\left\|x_{m j}-c_{m j}\right\|_{F}^{2}
$$

Figure 3.2 $\mathrm{b}$ shows an example of the behavior of both data error and the ACE, when the data set on Figure 3.2 -a is clustered from $m=1 \ldots, 30$. As can be seen from this figure, the minimum of ACE happens when $m=9$ which is the $\mathrm{CNC}$ and data error is evidently a decreasing function of $m$.

\section{2 k-MACE Approach}

The observed average central error $z_{s_{m}}$ is a sample of the random variable $Z_{s_{m}}$.

Adjoining (3.11) and (3.1), $Z_{s_{m j}}$ can be written as follows: (refer to Appendix 1 for details):

$$
Z_{s_{m j}}=\left\|A_{m j} \bar{c}_{m j}\right\|_{F}^{2}+\frac{1}{n_{m j}} \sum_{i=1}^{n_{m j}} \hat{W}_{x_{m j}^{i}} \hat{W}_{x_{m j}^{i}}^{T}+\frac{1}{n_{m j}} \sum_{i \neq k}^{n_{m j}} \hat{W}_{x_{m j}^{i}} \hat{W}_{x_{m j}^{T}}^{T}
$$

where

$$
A_{m j}=\left[\begin{array}{ccc}
1-\frac{1}{n_{m j}} & \ldots & \frac{-1}{n_{m j}} \\
\frac{-1}{n_{m j}} & 1-\frac{1}{n_{m j}} & \ldots \\
\ldots & \ldots & \ldots \\
\frac{-1}{n_{m j}} & \ldots & 1-\frac{1}{n_{m j}}
\end{array}\right]
$$



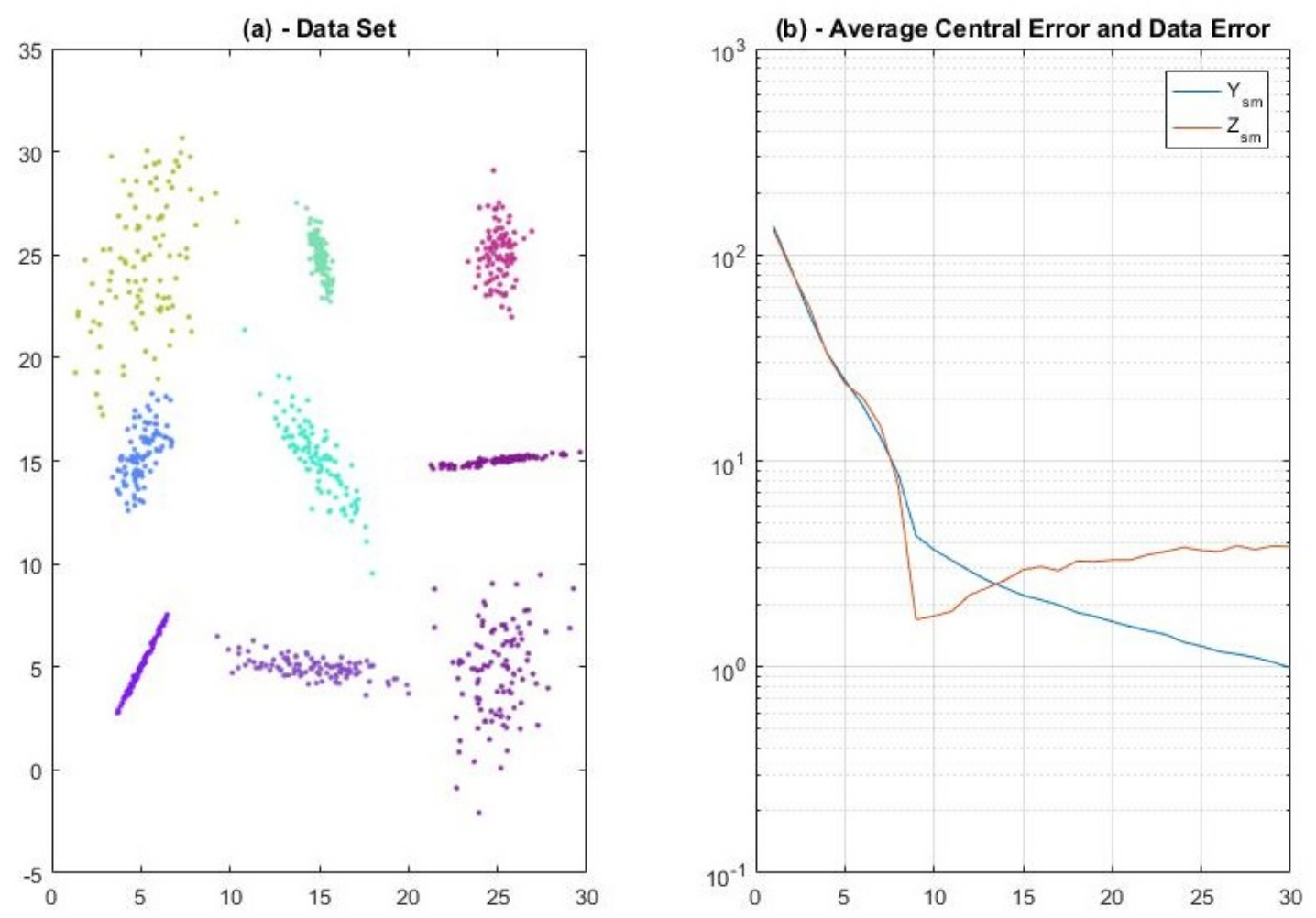

Figure 3.2: Example behavior of $Z_{s_{m}}$ and $Y_{s_{m}}$ on general Gaussian clusters

$\hat{W}_{x_{m j}^{i}}$ is a random vector that represents the variation of $i^{\text {th }}$ element of $x_{m j}$ around its true center.

We derive the expected value and the variance of $Z_{s_{m j}}$ (for details, refer to Appendix 1)

$$
\begin{gathered}
E\left[Z_{s_{m j}}\right]=\left\|A_{m j} \bar{c}_{x_{m j}}\right\|_{F}^{2}+\frac{1}{n_{m j}} \sum_{i=1}^{n_{m j}} \operatorname{tr}\left({\bigwedge_{x_{m j}^{i}}}\right) \\
\operatorname{Var}\left[Z_{s_{m j}}\right]=\frac{2}{n_{m j}{ }^{2}} \sum_{i=1}^{n_{m j}} \operatorname{tr}\left(\left({\bigwedge_{x_{m j}^{i}}}^{2}\right)+\frac{2}{n_{m j}{ }^{2}} \sum_{i \neq k}^{n_{m j}} \operatorname{tr}\left({\bigwedge_{x_{m j}^{i}}}_{\lambda_{x_{m j}^{k}}} \bar{x}\right.\right.
\end{gathered}
$$

where $\operatorname{tr}()$ is the trace function of a matrix and $\bar{\Lambda}_{x_{m j}^{i}}$ is the diagonalized eigenvalues of $\hat{W}_{x_{m j}^{i}}$ 's covariance matrix. 
Consequently, the expected value and variance of $Z_{s_{m}}$ is given below:

$$
\begin{gathered}
E\left[Z_{s_{m}}\right]=\frac{1}{N} \sum_{j=1}^{m} E\left[Z_{s_{m j}}\right] \\
\operatorname{Var}\left[Z_{s_{m}}\right]=\frac{1}{N^{2}} \sum_{j=1}^{m} \operatorname{Var}\left[Z_{s_{m j}}\right]
\end{gathered}
$$

From 3.17 , the expected value of $Z_{s_{m j}}$, has two terms. The first term, $\left\|A_{m j} \bar{c}_{m j}\right\|_{F}^{2}$, is a function of the unknown true cluster center. This term is a decreasing function of $m$. The second term is a function of the cluster covariance and is monotonically proportional to $m$. As a result, there is a point in which $Z_{s_{m}}$ will reach its minimum value at a finite $m$. This is traditionally called the bias-variance trade-off 21-22.

Figure 3.3 shows an example of the behavior of $E\left[Z_{s_{m}}\right]$ and $\operatorname{Var}\left[Z_{s_{m}}\right]$ measured on the data set depicted on Figure 3.2 -a. It can be seen that the variance of $Z_{s m}$ is negligible as compared to its expected value. Because of this property, it can be concluded that the distribution of $Z_{s m}$ is highly dense around $E\left[Z_{s m}\right]$. However, the actual ACE, $z_{s_{m}}$, is only a sample of the random variable $Z_{s_{m}}$, knowing the pdf of $Z_{s_{m}}$ cannot give us directly the value of the $z_{s_{m}}$ but it can be used to derived the probabilistic bounds of $Z_{s_{m}}$.

Confidence probability $P_{1}$ is incorporated so that

$$
P\left(\left|E\left[Z_{s_{m}}\right]-z_{s_{m}}\right| \leq \beta_{N} \sqrt{\operatorname{var}\left[Z_{s_{m}}\right]}\right)=P_{1}
$$

$P_{1}$ represents the probability that the actual average central error $z_{s_{m}}$ is within $\pm \beta_{N} \sqrt{\operatorname{Var}\left[Z_{s_{m}}\right]}$ from $E\left[Z_{s_{m}}\right]$. Naturally, we would want the confidence probability $P_{1}$ to be as large as possible. In order to achieve such goal, it requires $\beta_{N}$ to be proportional 
to $N$.

We define the upper bound and the lower bound for $Z_{s_{m}}$, respectively.

$$
\begin{aligned}
& \overline{Z_{s_{m}}}=E\left[Z_{s_{m}}\right]+\beta_{N} \sqrt{\operatorname{var}\left[Z_{s_{m}}\right]} \\
& \underline{Z_{s_{m}}}=E\left[Z_{s_{m}}\right]-\beta_{N} \sqrt{\operatorname{var}\left[Z_{s_{m}}\right]}
\end{aligned}
$$

such that given confidence probability $P_{1}$

$$
\underline{Z_{s_{m}}} \leq z_{s_{m}} \leq \overline{Z_{s_{m}}}
$$

The estimated CNC is found by finding $m$ where minimum of ACE's upper bound occurs.

$$
\hat{m}=\arg \min _{m}\left(\overline{Z_{s_{m}}}\right)
$$

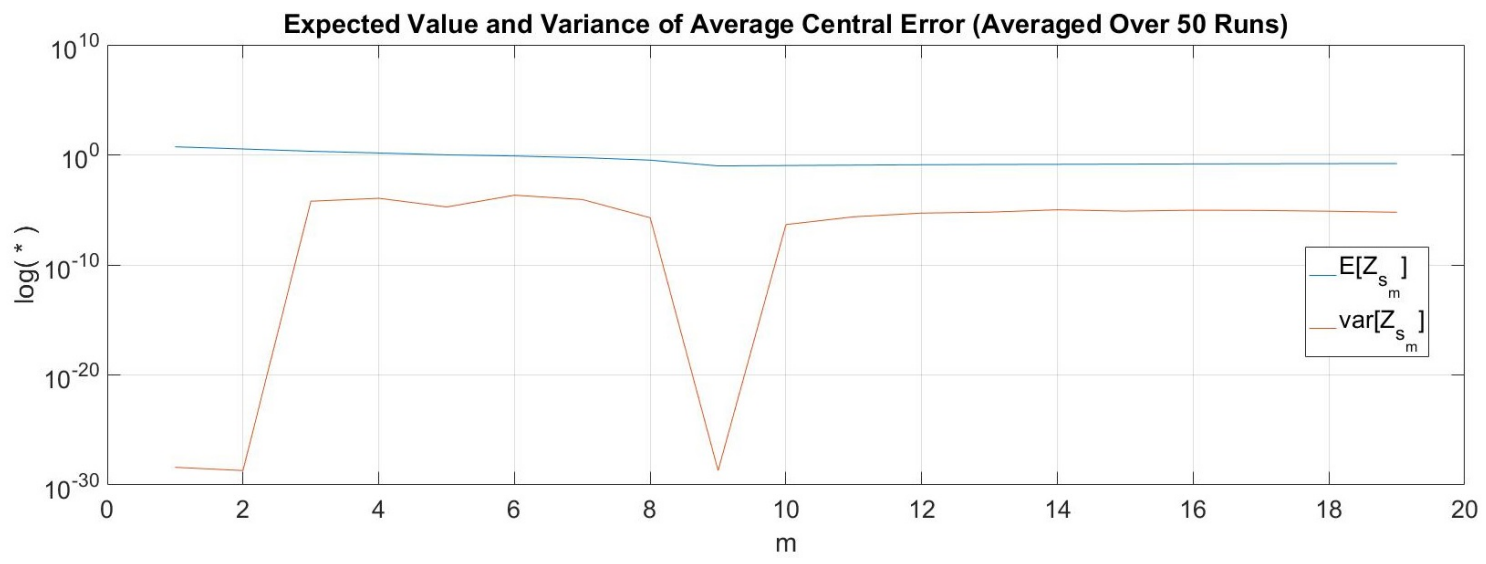

Figure 3.3: Expected value and variance of $Z_{s_{m}}$ for a range of $m$ derived from data set depicted on Figure 3.2 -a

The upper-bound of ACE is derived using the expected value of ACE which can only 
be solved if one knows the true cluster center. In the following section, we would show how to estimate the unknown term, $\left\|A_{m j} \bar{c}_{x_{m j}}\right\|_{F}^{2}$, from the data error.

\subsubsection{Estimating $\left\|\mathrm{A}_{\mathrm{mj}} \overline{\mathbf{c}}_{\mathrm{x}_{\mathrm{mj}}}\right\|_{\mathrm{F}}^{2}$}

The obseved data error $y_{s m}$ is a sample of the random variable $Y_{s m}$. The data error is the average distance of all the elements on the data set to its estimated cluster center.

Combining equations (3.1) and (3.14), (refer to Appendix 2 for details)

$$
\begin{array}{r}
Y_{s_{m j}}=\left\|A_{m j} \bar{c}_{x_{m j}}\right\|_{F}^{2}+\frac{n_{m j}-1}{n_{m j}} \sum_{i=1}^{n_{m j}} \hat{W}_{x_{m j}^{i}} \hat{W}_{x_{m j}^{i}}^{T}-\frac{1}{n_{m j}} \sum_{i \neq k}^{n_{m j}} \hat{W}_{x_{m j}^{i}} \hat{W}_{x_{m j}^{k}}^{T} \\
2 \sum_{i=1}^{n_{m j}} \bar{c}_{x_{m j}^{i}} \hat{W}_{x_{m j}^{i}}^{T}-\frac{2}{n_{m j}} \sum_{d^{\prime}=1}^{d}\left(\sum_{i=1}^{n_{m j}} \hat{W}_{x_{m j}^{i}}^{d^{\prime}} \sum_{i=1}^{n_{m j}} \bar{c}_{x_{m j}^{i}}^{d^{\prime}}\right)
\end{array}
$$

where $\hat{W}_{x_{m j}^{i}}^{d^{\prime}}$ and $\bar{c}_{x_{m j}^{i}}^{d^{\prime}}$ are the variation and the true center of the $i^{t h}$ element of $x_{m j}$ in the $d^{\prime}$ component, respectively.

Similar to $Z_{s_{m j}}$, the cluster compactness is also a function of the random vector $\hat{W}_{x_{m j}^{i}}$, we derived its expected value and variance (refer to Appendix 2 for details).

$$
\begin{gathered}
E\left[Y_{s_{m j}}\right]=\left\|A_{m j} \bar{c}_{x_{m j}}\right\|_{F}^{2}+\frac{\left(n_{m j}-1\right)}{n_{m j}} \sum_{i=1}^{n_{m j}} \operatorname{tr}\left({\bigwedge_{x_{m j}^{i}}}\right) \\
\operatorname{Var}\left[Y_{s_{m j}}\right]=\frac{2\left(n_{m j}-1\right)^{2}}{n_{m j}^{2}} \sum_{i=1}^{n_{m j}} \operatorname{tr}\left(\left(\bar{\wedge}_{x_{m j}^{i}}\right)^{2}\right)+\frac{1}{n_{m j}^{2}} \sum_{i \neq k}^{n_{m j}} \operatorname{tr}\left(\bar{\wedge}_{x_{m j}^{i}} \bar{\wedge}_{x_{m j}^{k}}\right) \\
\frac{4}{d n_{m j}}\left\|A_{m j} \bar{c}_{x_{m j}}\right\|_{F}^{2} \sum_{i=1}^{n_{m j}} \operatorname{tr}\left(\bar{\wedge}_{x_{m j}^{i}}\right)
\end{gathered}
$$


The expected value and variance of $Y_{s_{m}}$ is given below:

$$
\begin{gathered}
E\left[Y_{s_{m}}\right]=\frac{1}{N} \sum_{j=1}^{m} E\left[Y_{s_{m j}}\right] \\
\operatorname{Var}\left[Y_{s_{m}}\right]=\frac{1}{N^{2}} \sum_{j=1}^{m} \operatorname{Var}\left[Y_{s_{m j}}\right]
\end{gathered}
$$

Depicted on Figure 3.4 is an example of the behavior of the expected value and the variance $Y_{s_{m}}$ as a function of $m$.

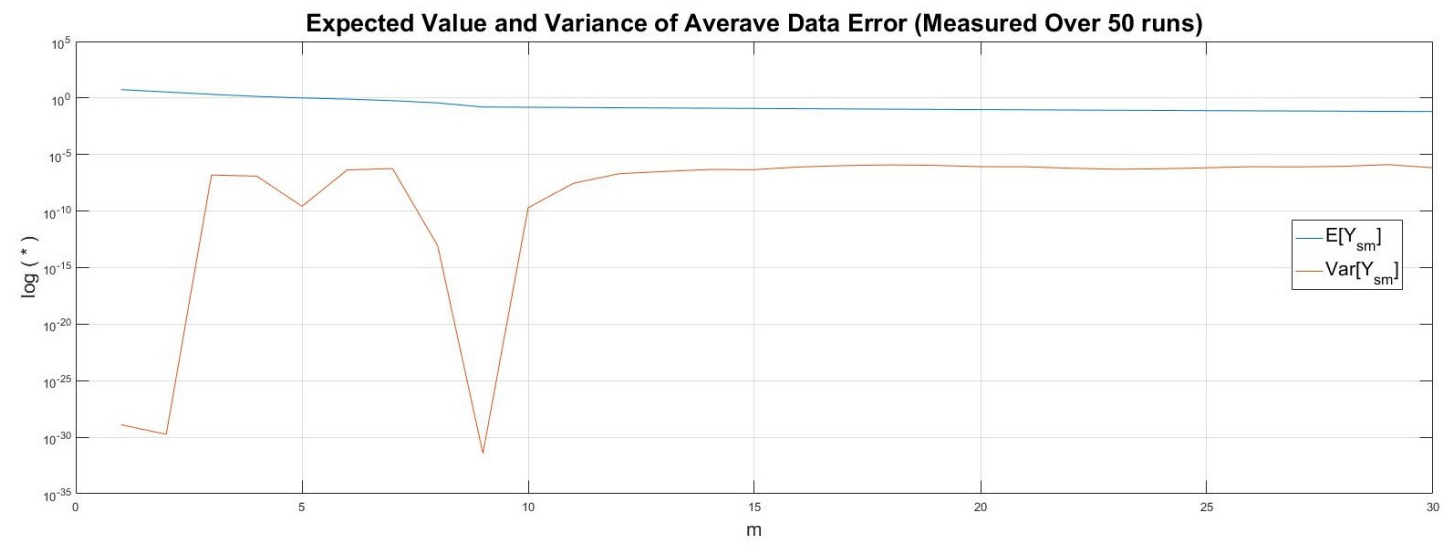

Figure 3.4: Expected value and variance of $Y_{s_{m}}$ for a range of $m$ derived from data set S1 depicted on figure 1a

We use the pdf of $Y_{s_{m j}}$ to provide confidence region for $\left\|A_{m j} \bar{c}_{x_{m j}}\right\|_{F}^{2}$. Given the validation probability $P_{2}$ such that,

$$
P\left(\left|E\left[Y_{s_{m}}\right]-y_{s_{m}}\right| \leq \alpha_{N} \sqrt{\operatorname{var}\left[Y_{s_{m}}\right]}\right)=P_{2}
$$

we can provide bounds for $\left\|A_{m j} \bar{c}_{m j}\right\|_{F}^{2}$ through probabilistic validation. Validation of $\left\|A_{m j} \bar{c}_{m j}\right\|_{F}^{2}$ is such that $y_{s_{m j}}$ is in the neighborhood of $Y_{s_{m j}}$ 's mean with a probability 
of $P_{2}$. Similar to $P_{1}$, we would want the validation probability $P_{2}$ to be large since this will give us bigger confidence region for $y_{s_{m j}}$. In order to achieve this, $\alpha_{N}$ should be proportinal to $N$.

To find the upper bound of $\left\|A_{m j} \bar{c}_{x_{m j}}\right\|_{F}^{2}$, we should solve for the following inequality:

$$
E\left[Y_{s_{m j}}\right]-\alpha_{N} \sqrt{\operatorname{var}\left[Y_{s_{m j}}\right]} \leq y_{s_{m j}}
$$

Plugging-in 3.27 and 3.28 to equation 3.32, and letting $\left\|A_{m j} \bar{c}_{x_{m j}}\right\|_{F}^{2}=m_{l j}, m_{w j}=$ $\frac{\left(n_{m j}-1\right)}{n_{m j}} \sum_{i=1}^{n_{m j}} \operatorname{tr}\left(\bar{\wedge}_{x_{m j}^{i}}\right)$, we get

$$
\alpha_{N} \sqrt{\frac{2\left(n_{m j}-1\right)^{2}}{n_{m j}^{2}} \sum_{i=1}^{n_{m j}} \operatorname{tr}\left(\left(\bar{\wedge}_{x_{m j}^{i}}\right)^{2}\right)+\frac{2}{n_{m j}^{2}} \sum_{i \neq k}^{n_{m j}} \operatorname{tr}\left({\bigwedge_{s^{i}}}_{x_{m j}} \bar{\wedge}_{x_{m j}^{k}}\right)+m_{l j} \frac{4}{d n_{m j}} \sum_{i=1}^{n_{m j}} \operatorname{tr}\left(\bar{\wedge}_{x_{m j}^{i}}\right)}
$$

To solve for the boundary, (i.e. when two sides of the inequality are equal), we square both sides of the equation

$$
\begin{gathered}
m_{l j}^{2}+m_{l j}\left(2\left(m_{w j}-y_{s_{m j}}\right)-\alpha_{N}^{2} \frac{4}{d n_{j}} \sum_{i=1}^{n_{m j}} \operatorname{tr}\left(\bar{\wedge}_{x_{m j}^{i}}\right)\right)+ \\
{\left[\left(m_{w j}-y_{s_{m j}}\right)^{2}-\alpha_{N}^{2}\left(\frac{2\left(n_{m j}-1\right)^{2}}{n_{m j}^{2}} \sum_{i=1}^{n_{m j}} \operatorname{tr}\left(\left(\bar{\wedge}_{x_{m j}^{i}}\right)^{2}\right)+\frac{2}{n_{m j}^{2}} \sum_{i \neq k}^{n_{m j}} \operatorname{tr}\left(\bar{\wedge}_{x_{m j}^{i}} \bar{\wedge}_{x_{m j}^{k}}\right)\right)\right]=0}
\end{gathered}
$$

(3.34) is a quadratic equation in terms of $m_{l j}$. Solving for the roots of $m_{l j}$ can give us both the upper-bound and the lower-bound, denoted by $\overline{m_{l j}}$ and $\underline{m_{l j}}$, respectively. The higher root of $m_{l j}$ is considered its upper bound. Note that the roots $m_{l j}$ is only a 
function of the observed data compactness $y_{s_{m}}, \alpha_{N}$, and the covariance of random vector $\hat{W}_{x_{m j}^{i}}, \bar{\wedge}_{x_{m j}^{i}}$.

After solving for the upper-bound of $m_{l j}$, we substitute this to (3.17), such that $m_{l j}=\overline{m_{l j}}$ in order to solve for the expected value of ACE.

$$
\begin{gathered}
E\left[Z_{s_{m j}}\right]=\overline{m_{l j}}+\frac{1}{n_{j}} \sum_{i=1}^{n_{j}} \operatorname{tr}\left(\bar{\wedge}_{x_{m j}^{i}}\right) \\
\operatorname{Var}\left[Z_{s_{m j}}\right]=\frac{2}{n_{m j}^{2}} \sum_{i=1}^{n_{m j}} \operatorname{tr}\left(\left({\bigwedge_{x_{m j}^{i}}}^{2}\right)+\frac{2}{n_{m j}{ }^{2}} \sum_{i \neq k}^{n_{m j}} \operatorname{tr}\left({\bigwedge_{x_{m j}^{i}}}_{\wedge_{x_{m j}^{k}}}\right)\right.
\end{gathered}
$$

Plugging in (3.35) to $(3.19)$ and $(3.36)$ to $(3.20)$, we can solve for the expected value and variance of the overall average central error. The upper-bound of the overall average central error $\overline{Z_{s_{m}}}$ can then be solve using 3.22 . ${ }_{4}^{4}$

Figure 3.5 shows the actual $Z_{s_{m}}$ and its estimated bounds as well $m_{l}$ as a function of $m$. The actual $Z_{s_{m}}$ and actual $m_{l}$ shown on this figure is calculated using the true cluster center. 5. The upper bound and the lower bound of both $Z_{s_{m}}$ and $\left\|A_{m j} \bar{c}_{x_{m j}}\right\|_{F}^{2}$ are calculated only using the observable cluster compactness as well as an estimate of $\bar{\wedge}_{x_{m j}^{i}}$ for $i=1,2 . ., N$.

\subsection{Estimating $\bar{\wedge}_{x_{i}}$}

The upper-bound of the average central error is estimated by relating the expected value and the variance of the observable data error to that of the unobservable ACE. And as seen from (3.17), (3.18), (3.27), and (3.28), these statistics are functions not only of the

\footnotetext{
${ }^{4}$ The ACE lower-bound, $Z_{s_{m}}$, can be solved by using $m_{l j}$ instead of $\overline{m_{l j}}$ in 3.35 .

${ }^{5} m_{l}$ is equivalent to $\frac{1}{N} \sum_{j=1}^{m}\left\|A_{m j} \bar{c}_{x_{m j}}\right\|_{F}^{2}$. The upper-bound of this term is solved by summation of all $\overline{m_{l j}}$ such that $\overline{m_{l}}=\frac{1}{N} \sum_{j=1}^{m} \overline{m_{l j}}$
} 

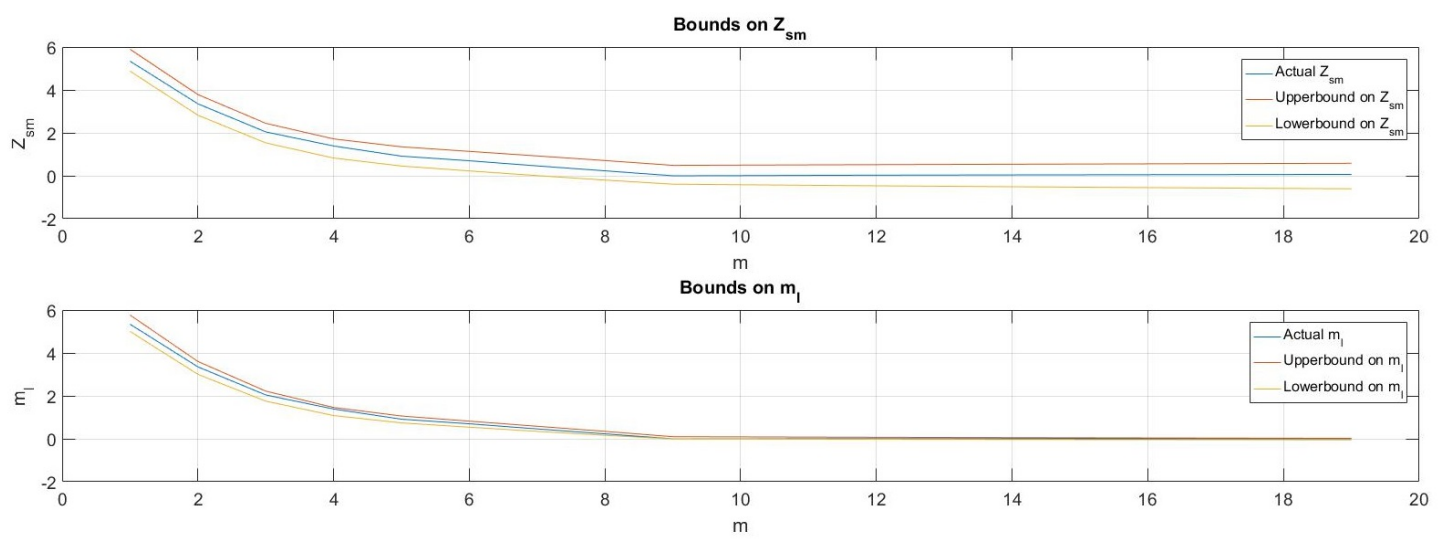

Figure 3.5: Behavior of $Z_{s_{m}}$ and $m_{l}$ and its derived bounds derived from data set depicted in Figure 3.2 -a

unknown true data center $\left\|A_{m j} \bar{c}_{m j}\right\|_{F}^{2}$ but as well as the unknown data covariance $\bar{\bigwedge}_{x_{m j}^{i}}$. Consider figure 3.6 which shows clustering result when the data is split into $m$ clusters such that $m>\bar{m}$. In the case when $m=4$, we have 4 clusters denoted by the colors red, purple, blue and yellow. It can be observed that in this case, a single cluster is further divided into multiple clusters. Examining the 1st cluster (red cluster, $j=1$ ) in $m=4$ clustering, we have

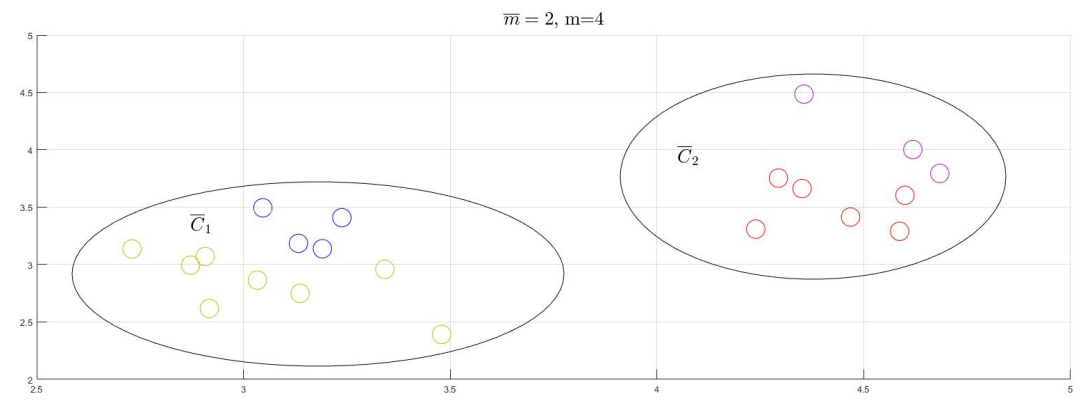

Figure 3.6: Clustering result when $m>\bar{m}$ (here, $\bar{m}=2, d=2, N=21$ ) 


$$
\begin{array}{r}
Y_{s_{m j}}=\left\|x_{m j}-c_{m j}\right\|_{F}^{2} \\
Y_{s_{m j}}=\left\|x_{m j}-B_{m j} x_{m j}\right\|_{F}^{2}
\end{array}
$$

where $B_{m j}$ is an averaging matrix

$$
B_{m j}=\left[\begin{array}{ccc}
\frac{1}{n_{m j}} & \cdots & \frac{1}{n_{m j}} \\
\frac{1}{n_{m j}} & \frac{1}{n_{m j}} & \cdots \\
\cdots & \cdots & \cdots \\
\frac{1}{n_{m j}} & \cdots & \frac{1}{n_{m j}}
\end{array}\right]
$$

Notice that all members of the red clusters belongs to the true cluster $\bar{C}_{2}$.

$$
Y_{s_{m j}}=\left\|\left(\bar{c}_{2}+\bar{W}_{X_{i}}\right)-B_{m j}\left(\bar{c}_{2}+\bar{W}_{X_{i}}\right)\right\|_{F}^{2}
$$

Since $B_{m_{m j}} \bar{c}_{2}=\bar{c}_{2}$

$$
\begin{gathered}
Y_{s_{m j}}=\left\|A_{m j} \bar{W}_{X_{i}}\right\|_{F}^{2} \\
Y_{s_{m j}}=\frac{n_{m j}-1}{n_{m j}} \sum_{i=1}^{n_{m j}} \bar{W}_{X_{i}} \bar{W}_{X_{i}}^{T}-\frac{1}{n_{m j}} \sum_{i \neq k}^{n_{m j}} \bar{W}_{X_{i}} \bar{W}_{X_{k}}^{T}
\end{gathered}
$$

From 3.41, it can be noticed that when $m>\bar{m}$, the cluster compactness $Y_{s_{m j}}$ becomes only a function of the random vector $\overline{W_{X_{i}}}$. Therefore, in order to estimate the unknown data covariance, we determine an initial estimate of number of cluster, $\hat{m}^{0}$, such that $\hat{m}^{0}>\bar{m}$. Cluster the data set with $\hat{m}^{0}$ clusters using Gaussian Mixuture Model 
(GMM) clustering, and finally using this clustering solution to obtain an estimate of $\hat{\wedge}_{x_{i}}$. The following process is explained in detail in the following:

Let $\hat{C}_{1}^{0}, \hat{C}_{2}^{0}, \ldots, \hat{C}_{\hat{m}^{0}}^{0}$ be the $\hat{m}^{0}$ clusters determined by GMM clustering. Calculate the covariance of each cluster $\hat{C}_{j}^{0}$ and take its eigenvalues and form a diagonal matrix whose elements are these eigenvalues. We denote these matrices by $\wedge_{1}, \wedge_{2}, \ldots, \wedge_{\hat{m}^{0}}$, with $\wedge_{j}$ corresponding to $\hat{C}_{j}^{0}$. For each element $x_{i} \in\left[x_{1}, x_{2}, \ldots, x_{N}\right]$, we determine its cluster membership and

$$
\hat{\wedge}_{x_{i}}=\wedge_{j} \mid x_{i} \in \hat{C}_{j}^{0}
$$

\subsubsection{Obtaining $\hat{\mathbf{m}}^{0}$}

From figure 3.4, it can be observed that as soon as $m>\bar{m}, Y_{s_{m}}$ stops decreasing drastically. By taking the point $m$ in which $Y_{s_{m}}$ does not show significant changes, we would have an idea of when $m$ surpasses $\bar{m}$. Although $y_{s_{m}}$ cannot give us a good estimate of the correct number of clusters, we can examine its behavior as a function of $m$ and use it to obtain $\hat{m}^{0}$. Note that it was stated that $\hat{m}^{0}$ can take any $m$ value as long as $m>\hat{m}$.

To obtain $\hat{m}^{0}$, one would need to locate the point in which $\log \left(y_{s m}\right)$ starts showing insignificant changes. To do this, we take a derivative of $\log \left(y_{s m}\right)$ with respect to $m$

$$
\begin{gathered}
y_{s_{m}}^{\prime}=\log \left(y_{s_{m}}\right) \\
\frac{d\left(y_{s_{m}}^{\prime}\right)}{d m}=y_{s_{m}}^{\prime} *\left[\begin{array}{ll}
-1 & 1
\end{array}\right] \\
\frac{d^{\prime}\left(y_{s m}^{\prime}\right)}{d m}=\frac{\frac{d\left(y_{s m}^{\prime}\right)}{d m}}{\max \left(\frac{d\left(y_{s m}^{\prime}\right)}{d m}\right)}
\end{gathered}
$$

where $(a * b)$ is convolution of vectors $a$ and $b$. We also normalize $\frac{d\left(y_{s m}^{\prime}\right)}{d m}$ so that its 
maximum value is 1 .

$$
\hat{m}^{0}=\min \left(\arg _{m}\left(\frac{d^{\prime}\left(y_{s_{m}}^{\prime}\right)}{d m} \leq T\right)\right)
$$

The term $\arg _{m}\left(\frac{d Y_{s_{m}}}{d m}<=T\right)$ is obtained by taking all the $m$ values for which $\frac{d^{\prime}\left(y_{s m}^{\prime}\right)}{d m}<$ T. $T$ is a hard threshold that should have a value close to 0 . In our algorithm, we use $T=0.1$.

\subsubsection{Why Use GMM for Estimation of Covariances}

GMM is used in calculating the covariance since it is better in detecting clusters that are general Gaussian distributed compared to k-means. The crisp clustering assignment of element $x_{i}$ in k-means is based on the $l 2$-norm between $x_{i}$ and the cluster centers. Due to this, k-means is only capable of detecting clusters that are spheroidal. An example of this case is depicted on Figure $3.7 \mathrm{~b}$

From Figure 3.7a, notice that the even though the data is clustered with $m=10$, GMM clustering solution is able to provide 6 major clusters which are very close to the true clustering solution. Almost all of the elements of the data set belongs to these 6 major clusters which will allow for a more accurate estimation of covariances of random vectors $\bar{W}_{x_{i}}, i=[1, \ldots, N]$.

On the other hand, examining top right part of Figure 3.7b, we noticed the following: 1) There are 3 clusters, each cluster has an ellipsoidal shape but with different orientation. 2) k-means clustering solution grouped elements from different clusters forming loose clusters.

Because of the reasons stated above, using k-means clustering solution will get us unreliable estimate of the cluster covariance.

Although GMM gives a better clustering solution when dealing with data whose 


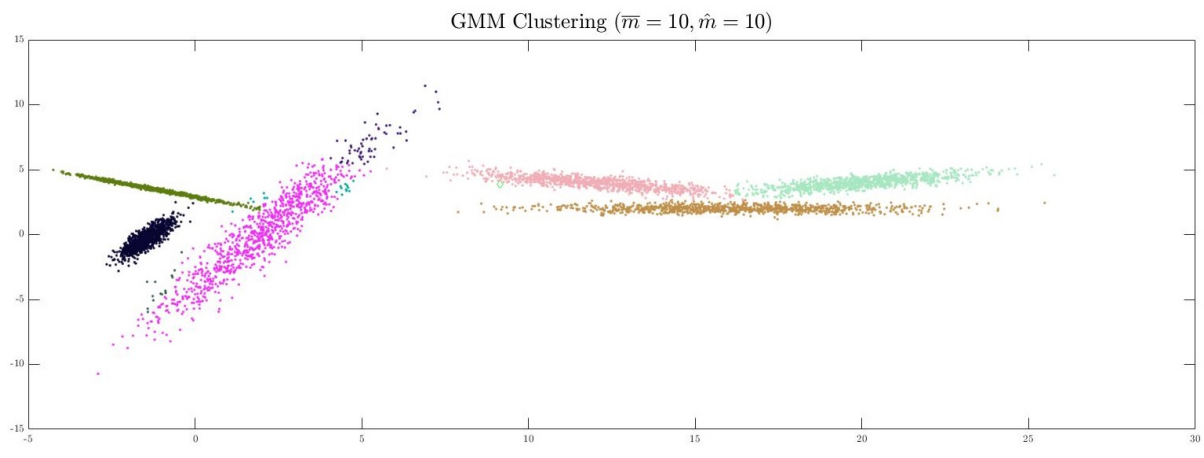

(a) GMM clustering $\left(\hat{m}^{0}=10, \bar{m}=6, d=2, N=6000\right)$

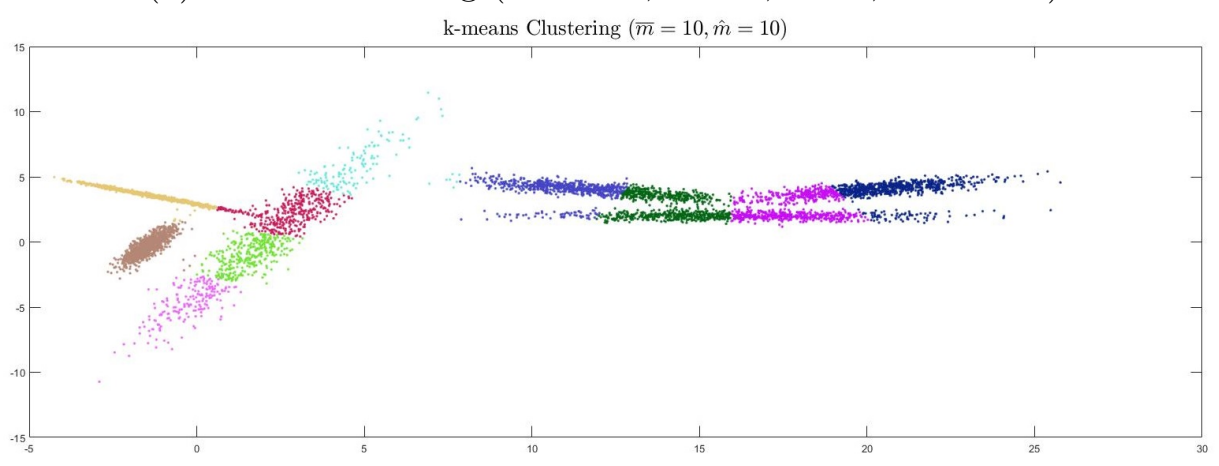

(b) k-means clustering $\left(\hat{m}^{0}=10, \bar{m}=6, d=2, N=6000\right)$

Figure 3.7: Clustering solution: GMM vs K-means

clusters have general Gaussian distribution, we are unable to use this in place of k-means for evaluations of $Z_{s_{m}}$ and $Y_{s_{m}}$. This is because GMM does not provide robust clustering solution when $m<\bar{m}$. This is supported by Figure 3.8. It can be noticed that both the average central error and the data error has huge variance when GMM is used instead of k-means. Our formulations assumes that the variance of these errors are much smaller compared to its expected value which is true for the case when k-means is used.

The clustering solution of GMM starts to become more robust when $m>\bar{m}$ (signified by low $Z_{s_{m}}$ and $Y_{s_{m}}$ variance), thus, we can use it to help us estimate $\bar{\Lambda}_{x_{i}}$. 


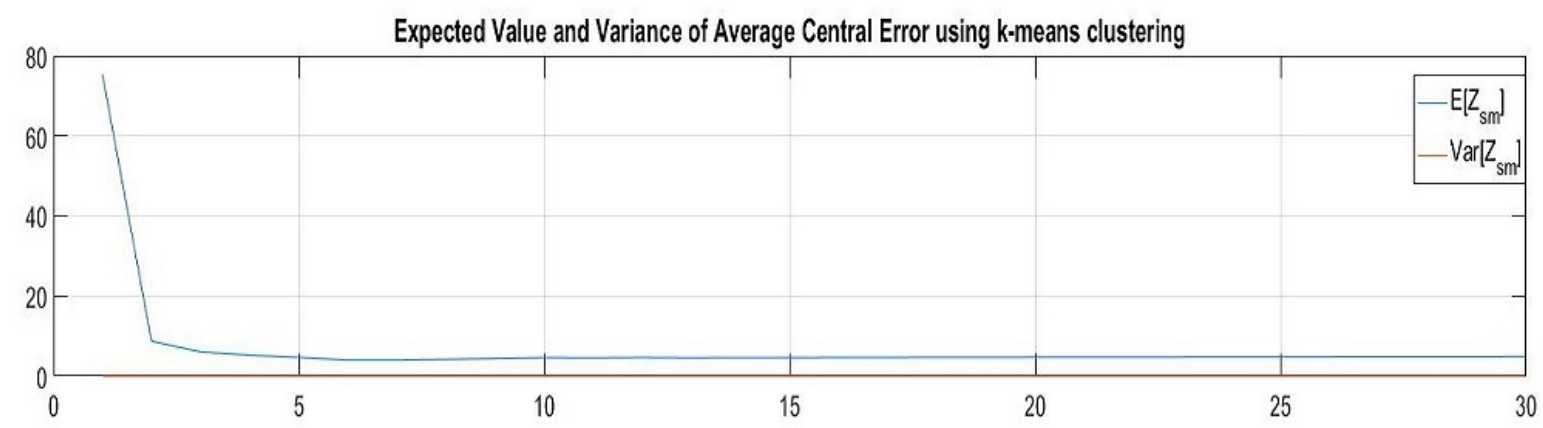

Expected Value and Variance of Average Central Error using GMM clustering

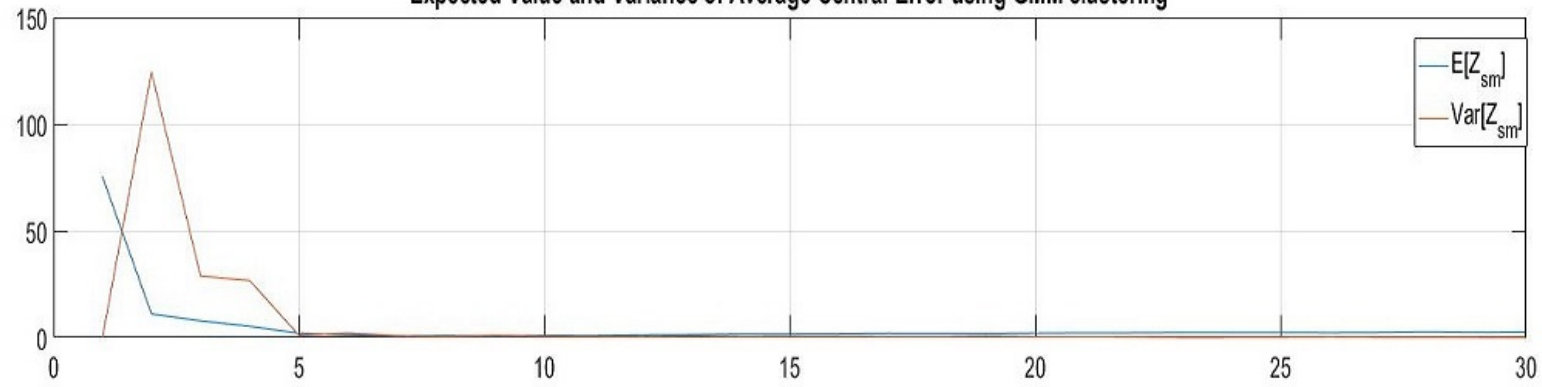

(a) Average central error on data set from Figure 3.2

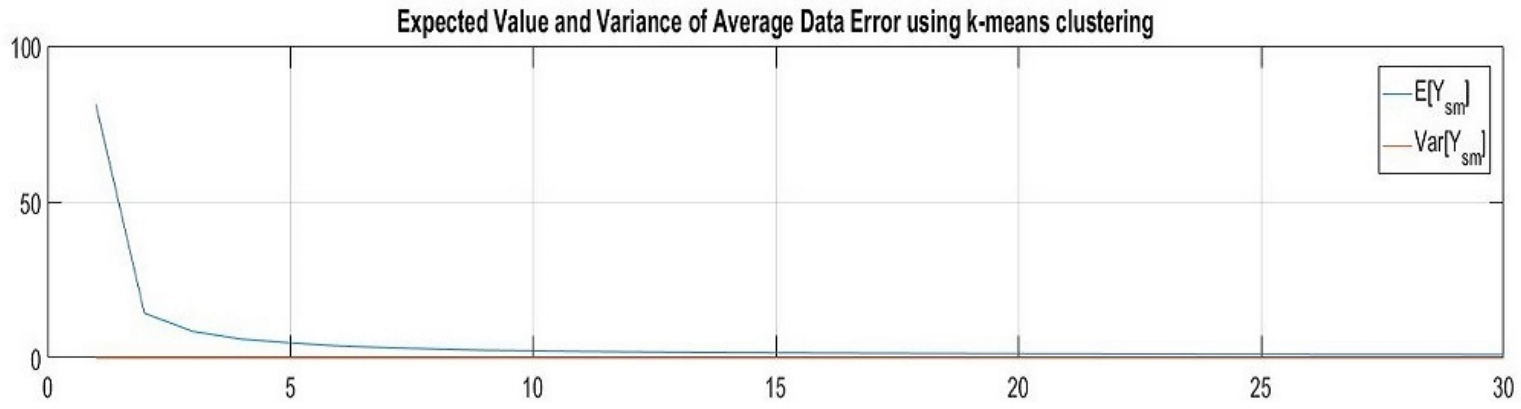

Expected Value and Variance of Average Data Error using GMM clustering

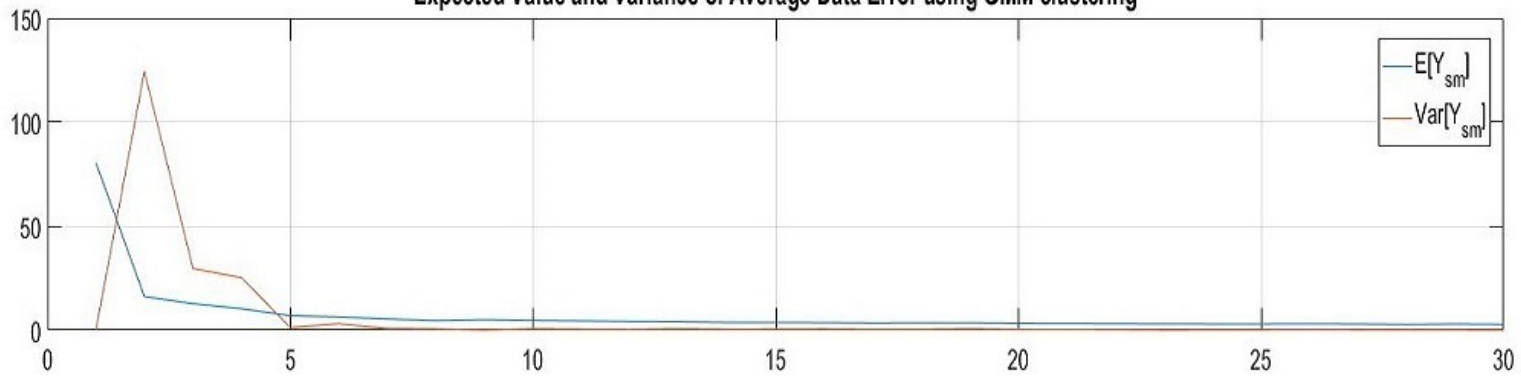

(b) Average data error on data set from Figure 3.2

Figure 3.8: Behavior of $Z_{s_{m}}$ and $Y_{s_{m}}$ : GMM vs K-means 


\subsection{Computational Complexity of k-MACE}

K-means computational complexity is $O(m N d l)$, where $N$ is the number of elements in the data set, $d$ is the dimension of the data, $m$ is the number of clusters that the data should be partitioned to and $l$ is the maximum number of iteration for k-means. $\mathrm{k}$ - MACE serves as a wrapper to k-means where we try to estimate the upperbound of $Z_{s_{m}}$ from the $y_{s_{m}}$ for each $m \in\left[m_{\min }, \ldots, m_{\max }\right]$. This means that for each $m$, we cluster the data into $m$ clusters using k-means which requires us to run k-means $m^{\prime}=m_{\max }-m_{\min }$ times. The estimation of $Z_{s_{m}}$ from $y_{s_{m}}$ does not impose a significant computational time on $\mathrm{k}-\mathrm{MACE}$. Therefore, $\mathrm{k}-\mathrm{MACE}$ computational complexity is given by:

$$
O\left(m^{\prime}\right) \times O(m N d l)
$$

where $m^{\prime}=m_{\max }-m_{\min }$.

The $\mathrm{k}-\mathrm{MACE}$ Algorithm is explained below. 


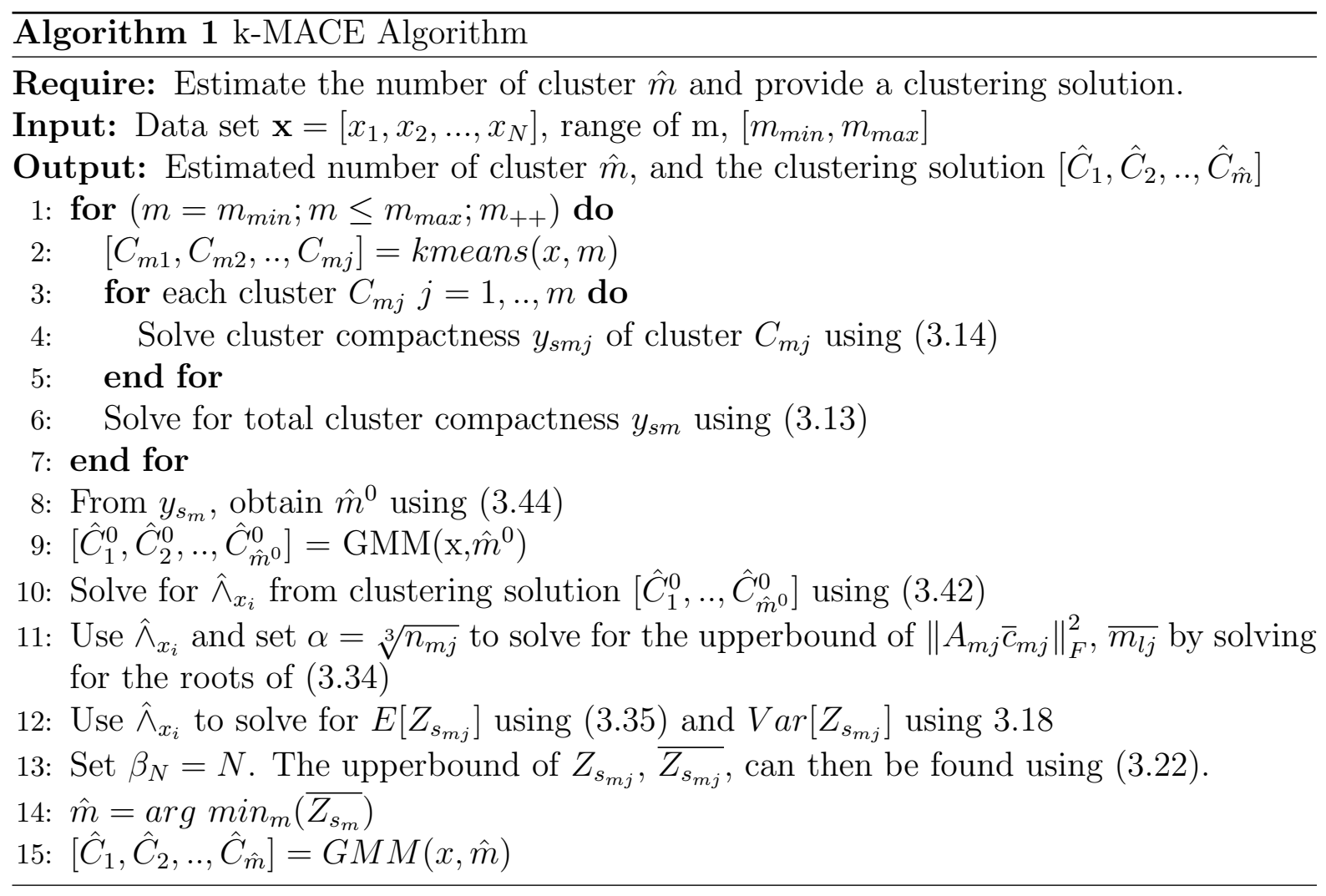




\section{Chapter 4}

\section{k-MACE Clustering $_{\sigma}$}

The proposed $\mathrm{k}$ - MACE method estimates the correct number of clusters through estimation of the average central error. Our formulations assumes that the data set is composed of clusters with possibly different covariance structure. In this section, we develop a special case of $\mathrm{k}$ - MACE for when the data set is composed of clusters that are independent and identically distributed IID and share the same variance. This method, denoted by $\mathrm{k}-\mathrm{MACE}_{\sigma}$, allows for simplification of the formulations of $\mathrm{k}-\mathrm{MACE}$.

This chapter is organized as follows: Section 4.1 shows how $\mathrm{k}-\mathrm{MACE}_{\sigma}$ is derived from $\mathrm{k}$ - MACE by having the assumption of IID clusters. Section 4.2 shows computational complexity of $\mathrm{k}-\mathrm{MACE}_{\sigma}$. Section 4.3 compares performance of $\mathrm{k}-\mathrm{MACE}$ and $\mathrm{k}-\mathrm{MACE}_{\sigma}$ for clusters that are IID. Section 4.4 offers a validation step that helps determine whether $\mathrm{k}-\mathrm{MACE}_{\sigma}$ is preferable than $\mathrm{k}-\mathrm{MACE}$ for estimating $\mathrm{CNC}$ of a given data set. Smart $\mathrm{k}-\mathrm{MACE}_{\sigma}$ clustering is proposed on section 4.5 that is an extension of $\mathrm{k}-\mathrm{MACE}_{\sigma}$ and has the capability of partitioning general Gaussian clusters. 
(a) - Data Set

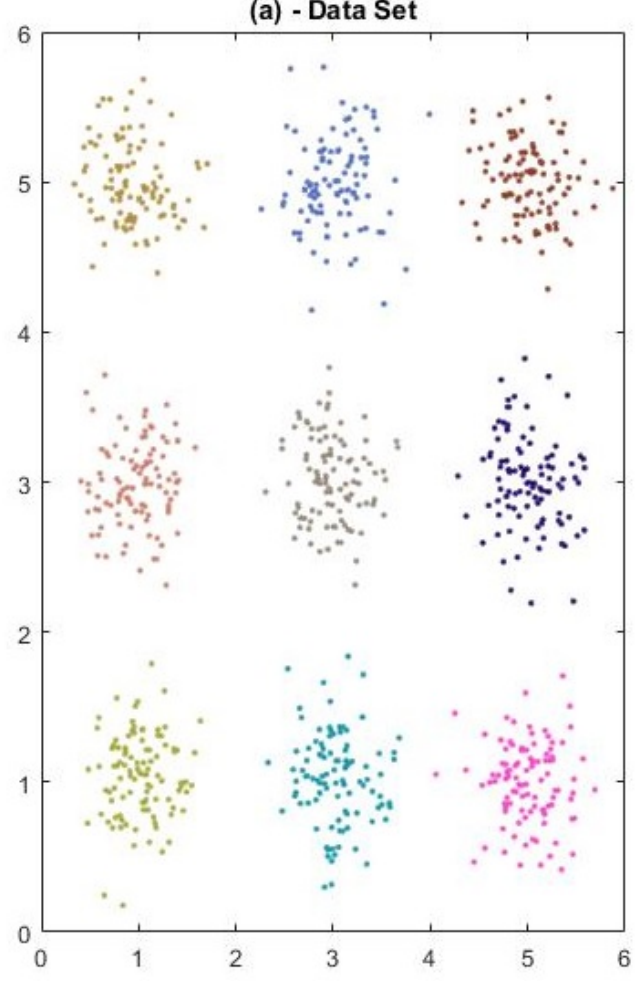

(b) - Average Central Error and Data Error

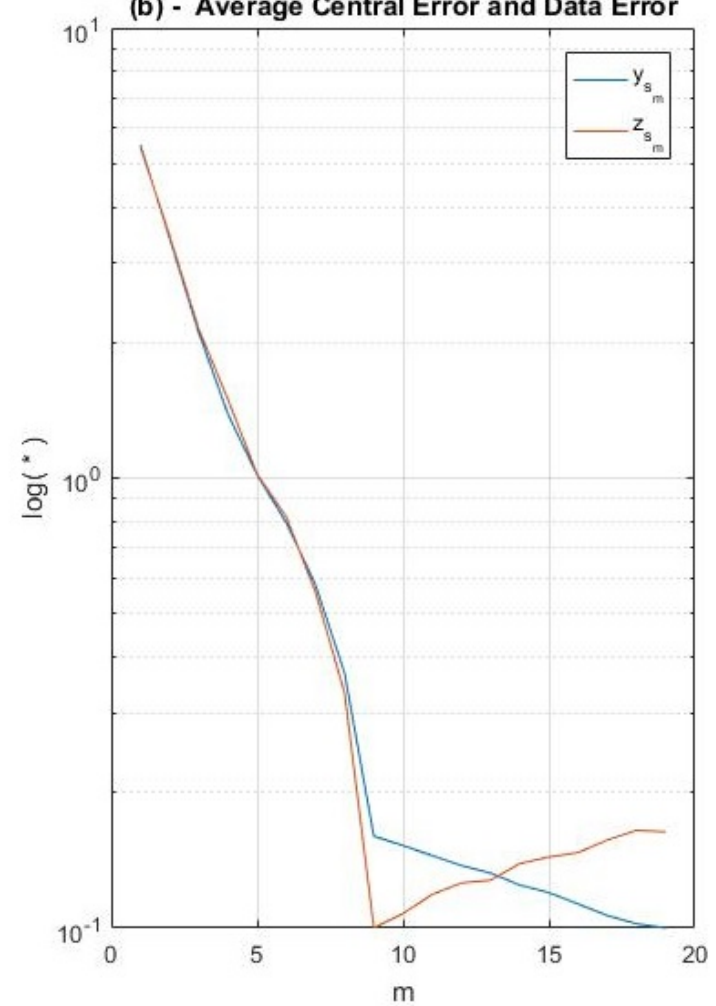

Figure 4.1: Example behavior of $Z_{s_{m}}$ and $Y_{s_{m}}$ on IID clusters

\section{$4.1 \mathrm{k}-\mathrm{MACE} \mathrm{E}_{\sigma} \equiv \mathrm{k}-\mathrm{MACE}$ When Clusters are IID}

Assuming that all clusters are IID around its center, it will imply that the variation of all the elements $\bar{W}_{X_{i}} \sim \mathcal{N}\left(0, \sigma_{w}^{2} \mathbf{I}_{d \times d}\right)$ for $i=1,2, \ldots, N$. An example of this type of data is depicted of Figure 4.1 a. For this case, we can directly solve for the upper-bound of $\frac{1}{N} \sum_{j=1}^{m}\left\|A_{m j} \bar{c}_{m j}\right\|_{F}^{2}$ from the total observed cluster compactness $y_{s_{m}}$ and from there, we can solve the upper-bound of the overall average central error $\overline{Z_{s_{m}}}$.

$$
y_{s_{m}} \rightarrow \overline{\frac{1}{N} \sum_{j=1}^{m}\left\|A_{m j} \bar{c}_{m j}\right\|_{F}^{2}} \rightarrow \overline{Z_{s_{m}}}
$$




\subsubsection{Average Central Error}

The expected value and the variance of $Z_{s_{m j}}$ can be simplified under the assumption that $\bar{W}_{X_{i}} \sim \mathcal{N}\left(0, \sigma_{w}^{2} \mathbf{I}_{d \times d}\right)$ for $i=1,,, N$ such that (see Appendix 1 for details)

$$
\begin{gathered}
E\left[Z_{s_{m j}}\right]=\left\|A_{m j} \bar{c}_{m j}\right\|_{F}^{2}+d \sigma_{w}^{2} \\
\operatorname{Var}\left[Z_{s_{m j}}\right]=2 d \sigma_{w}^{4}
\end{gathered}
$$

Applying equations (4.2) and (4.3) to (3.19) and (3.20) respectively, the expected value and variance of the overall average central error is given as follows:

$$
\begin{gathered}
E\left[Z_{s_{m}}\right]=\frac{1}{N} \sum_{j=1}^{m}\left\|A_{m j} \bar{c}_{m j}\right\|_{F}^{2}+\frac{d m \sigma_{w}^{2}}{N} \\
\operatorname{Var}\left[Z_{s_{m}}\right]=\frac{2 m d \sigma_{w}^{4}}{N^{2}}
\end{gathered}
$$

We can define the bounds on $Z_{s_{m}}$ using equations (4.4) and (4.5) and by letting $\beta_{N}=N$ (See Appendix 1 for details).

$$
\begin{aligned}
& \overline{Z_{s_{m}}}=\frac{1}{N} \sum_{j=1}^{m}\left\|A_{m j} \bar{c}_{m j}\right\|_{F}^{2}+\frac{m d \sigma_{w}^{2}}{N}+\sigma_{w}^{2} \sqrt{2 m d} \\
& \underline{\underline{Z_{s_{m}}}}=\frac{1}{N} \sum_{j=1}^{m}\left\|A_{m j} \bar{c}_{m j}\right\|_{F}^{2}+\frac{m d \sigma_{w}^{2}}{N}-\sigma_{w}^{2} \sqrt{2 m d}
\end{aligned}
$$

where $\overline{Z_{s_{m}}}$ and $\underline{Z_{s_{m}}}$ are the upper-bound and lower-bound of $z_{s_{m}}$, respectively. 


\subsubsection{Data Error}

Similarly, $Y_{s_{m}}$ can be simplified if we apply the assumption that $\bar{W}_{X_{i}} \sim \mathcal{N}\left(0, \sigma_{w}^{2} \mathbf{I}_{d \times d}\right)$ for $i=1,2, \ldots, N$. The expected value and variance of $Y_{s_{m j}}$ is given below

$$
\begin{gathered}
E\left[Y_{s_{m j}}\right]=\left\|A_{m j} \bar{c}_{m j}\right\|_{F}^{2}+d\left(n_{m j}-1\right) \sigma_{w}^{2} \\
\operatorname{Var}\left[Y_{s_{m j}}\right]=4 \sigma_{w}^{2}\left\|A_{m j} \bar{c}_{m j}\right\|_{F}^{2}+2 d\left(n_{m j}-1\right) \sigma_{w}^{4}
\end{gathered}
$$

The expected value and variance of the overall data error is given by:

$$
\begin{gathered}
E\left[Y_{s_{m}}\right]=\frac{1}{N} \sum_{j=1}^{m}\left\|A_{m j} \bar{c}_{m j}\right\|_{F}^{2}+\frac{d(N-m) \sigma_{w}^{2}}{N} \\
\operatorname{Var}\left[Y_{s_{m}}\right]=\frac{4 \sigma_{w}^{2}}{N^{2}} \sum_{j=1}^{m}\left\|A_{m j} \bar{c}_{m j}\right\|_{F}^{2}+\frac{2 d(N-m) \sigma_{w}^{4}}{N}
\end{gathered}
$$

Letting $m_{l}=\frac{1}{N} \sum_{j=1}^{m}\left\|A_{m j} \bar{c}_{m j}\right\|_{F}^{2}$ and $m_{w}=\frac{d(N-m) \sigma_{w}^{2}}{N}$, the bounds on $m_{l}$ are as follows (See Appendix 2 for details).

$$
\begin{aligned}
& \overline{m_{l}}=y_{s_{m}}-m_{w}+2 \frac{\alpha^{2} \sigma_{w}^{2}}{N}+k_{s m}(\alpha) \\
& \underline{m_{l}}=y_{s_{m}}-m_{w}+2 \frac{\alpha^{2} \sigma_{w}^{2}}{N}-k_{s m}(\alpha)
\end{aligned}
$$

and

$$
k_{s m}(\alpha)=\frac{2 \alpha \sigma_{w}}{\sqrt{N}} \sqrt{\frac{\alpha^{2} \sigma_{w}^{2}}{N}+y_{s_{m}}-\frac{1}{2} m_{w}}
$$

Equation (4.6) and (4.7) provides the bounds for $Z_{s_{m}}$ assuming that $m_{l}$ is known, in 
real applications however, this variable is unknown. To estimate the upper-bound and the lower-bound of $Z_{s_{m}}$, we combine equations (4.6) with 4.12) and (4.7) with 4.13) respectively.

$$
\begin{aligned}
& \overline{Z_{s_{m}}}=\overline{m_{l}}+\frac{m d \sigma_{w}^{2}}{N}+\sigma_{w}^{2} \sqrt{2 m d} \\
& \underline{Z_{s_{m}}}=\underline{m_{l}}+\frac{m d \sigma_{w}^{2}}{N}-\sigma_{w}^{2} \sqrt{2 m d}
\end{aligned}
$$

And similar to $\mathrm{k}-\mathrm{MACE}_{\sigma}$, the estimate of correct number of cluster is given by

$$
\hat{m}=\arg \min _{m}\left(\overline{Z_{s_{m}}}\right)
$$

\subsubsection{Estimating $\sigma_{\mathrm{w}}^{2}$}

As seen from Section 3.3, when $m>\hat{m}$, the data error becomes only a function of the random vector $\bar{W}_{X_{i}}$. Under the assumption that all clusters are IID with variance $\sigma_{w}^{2}$, the expected value and variance of $Y_{s_{m}}$ can be easily derived as follows (Refer to Appendix 2):

$$
\begin{gathered}
E\left[Y_{s_{m}}\right]=\frac{d(N-m)}{N} \sigma_{w}^{2} \quad \mid \quad m>\bar{m} \\
\operatorname{Var}\left[Y_{s_{m}}\right]=\frac{2 d(N-m)}{N^{2}} \sigma_{w}^{4} \quad \mid \quad m>\bar{m}
\end{gathered}
$$

For large values of $N$, the expected value of cluster compactness will be much higher compared to its variance. We can therefore assume that the observed cluster compactness $y_{s_{m}}$ is a good representation of $E\left[Y_{s_{m}}\right]\left(y_{s_{m}} \approx E\left[Y_{s_{m}}\right]\right)$. With this information, we would 
be able to solve for the variance.

$$
\hat{\sigma}_{w}^{2}=\sum_{m=\hat{m}^{0}}^{m_{\max }} \frac{N y_{s_{m}}}{d(N-m)}
$$

where $\hat{m}^{0}$ is obtained using the process explained on Section 3.3.1.

\subsection{Computational Analysis of k-MACE ${ }_{\sigma}$}

The computational complexity of $\mathrm{k}-\mathrm{MACE}_{\sigma}$ is similar to that of $\mathrm{k}-\mathrm{MACE}$. Although estimation of bounds on $z_{s_{m}}$ is simpler for $\mathrm{k}-\mathrm{MACE}_{\sigma}$, it is still the calculation of $y_{s_{m}}$, that gives majority of computational complexity. This being said, the computational complexity of $\mathrm{k}-\mathrm{MACE}_{\sigma}$ is also $O\left(m^{\prime}\right) \times O(m N d l)$. The over-all algorithm of $\mathrm{k}-\mathrm{MACE}_{\sigma}$ is described below.

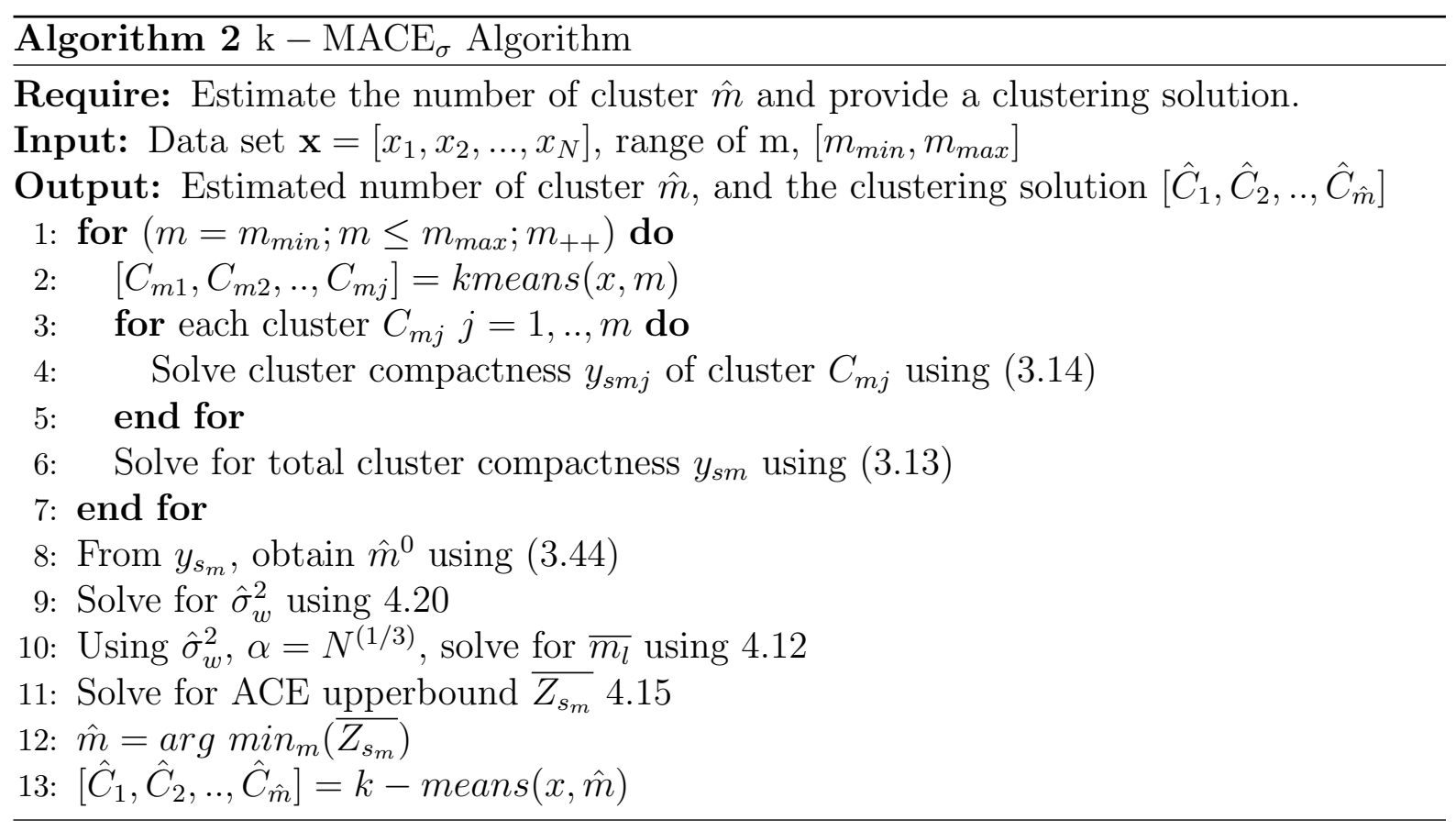




\subsection{An Example of k-MACE and k-MACE $\mathrm{H}_{\sigma}$ on Clus- ters That Are IID}

The motivation of this section is to show how $\mathrm{k}-\mathrm{MACE}_{\sigma}$ outperforms $\mathrm{k}-\mathrm{MACE}$ in an IID clustering setting. While both $\mathrm{k}-\mathrm{MACE}$ and $\mathrm{k}-\mathrm{MACE}_{\sigma}$ aims to look for the number of cluster that minimizes the average central error, k-MACE assumes that the data set is composed of clusters with general Gaussian distribution. On the other hand, the premise on $\mathrm{k}-\mathrm{MACE}_{\sigma}$ ' is that the data set contains clusters that are IID with uniform variance. Ultimately, this assumption causes the main difference between the $\mathrm{k}-\mathrm{MACE}$ and $\mathrm{k}-\mathrm{MACE}_{\sigma}$.

Consider the example from Figure 4.2 where the clusters are IID and has uniform variance and varying degrees of overlap between clusters. Evidently, the result from $\mathrm{k}-\mathrm{MACE}_{\sigma}$ clustering is closer to the correct solution while $\mathrm{k}-\mathrm{MACE}$ underestimated the correct number of cluster. It can be observed from Figure $4.2 \mathrm{~b}$ that touching IID clusters are interpreted as one cluster by $\mathrm{k}-\mathrm{MACE}$.

Recall from Section 3 that the ACE is composed of two components:

$$
E\left[Z_{s_{m j}}\right]=\left\|A_{m j} \bar{c}_{m j}\right\|_{F}^{2}+\frac{1}{n_{m j}} \sum_{i=1}^{n_{m j}} \operatorname{tr}\left(\bar{\bigwedge}_{x_{m j}^{i}}\right)
$$

The first term of 4.21 is a decreasing function of $m$ and is a function of the unknown true cluster center. The second term is a function of the true covariance of the cluster and increases as $m$ increases. For k-MACE, we rely on GMM clustering for the estimation of the true cluster covariance and use this estimate to provide ACE's upper bound. For D31 data set, GMM ended up combining touching IID clusters forming a less compact generally distributed cluster. This is depicted on Figure 4.3a. Due to this, the estimate of cluster covariance will be larger than the true covariance thus making the second term 


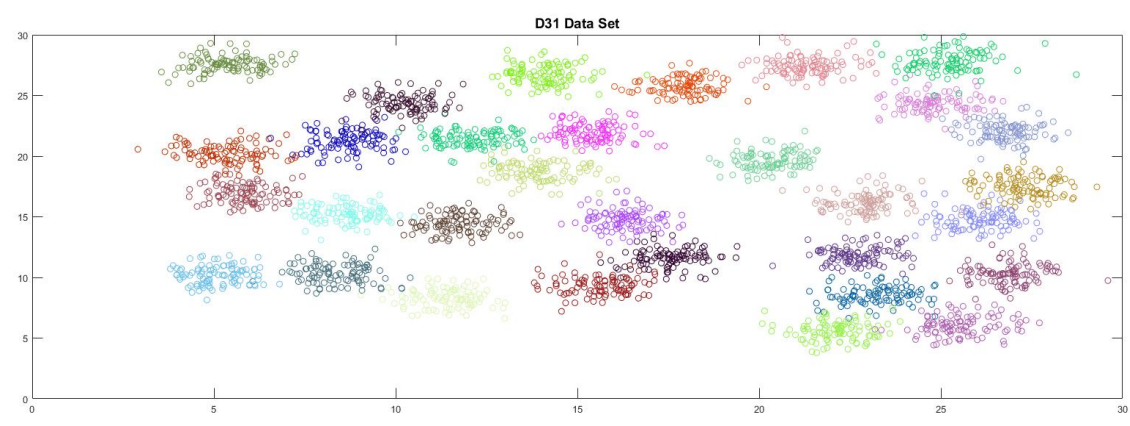

(a) Data Set ( $m=31$, IID clusters, uniform variance)

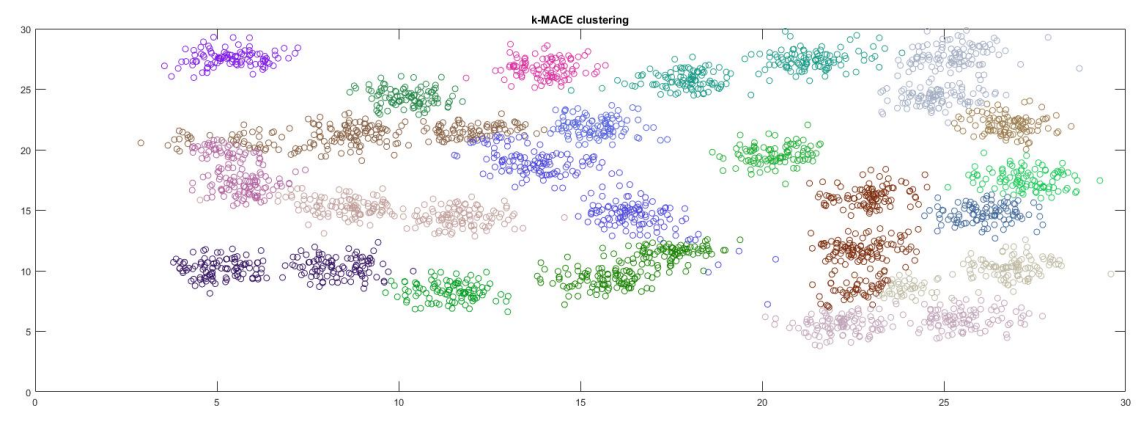

(b) k-MACE clustering $(\hat{m}=15)$

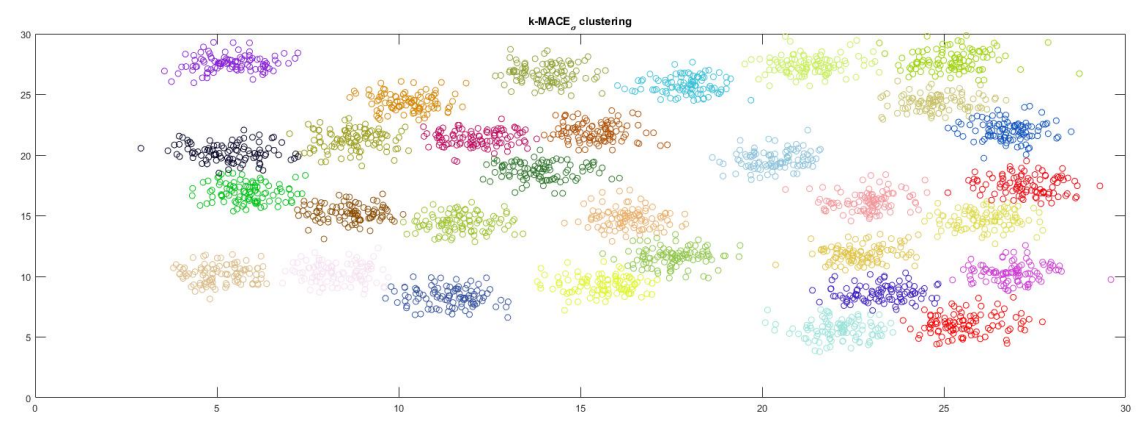

(c) $\mathrm{k}-\mathrm{MACE}_{\sigma}$ clustering $(\hat{m}=31)$

Figure 4.2: Example Clustering Solution: $\mathrm{k}-\mathrm{MACE}$ vs $\mathrm{k}-\mathrm{MACE}_{\sigma}$ on IID clusters increase faster as depicted on Figure 4.3b. 


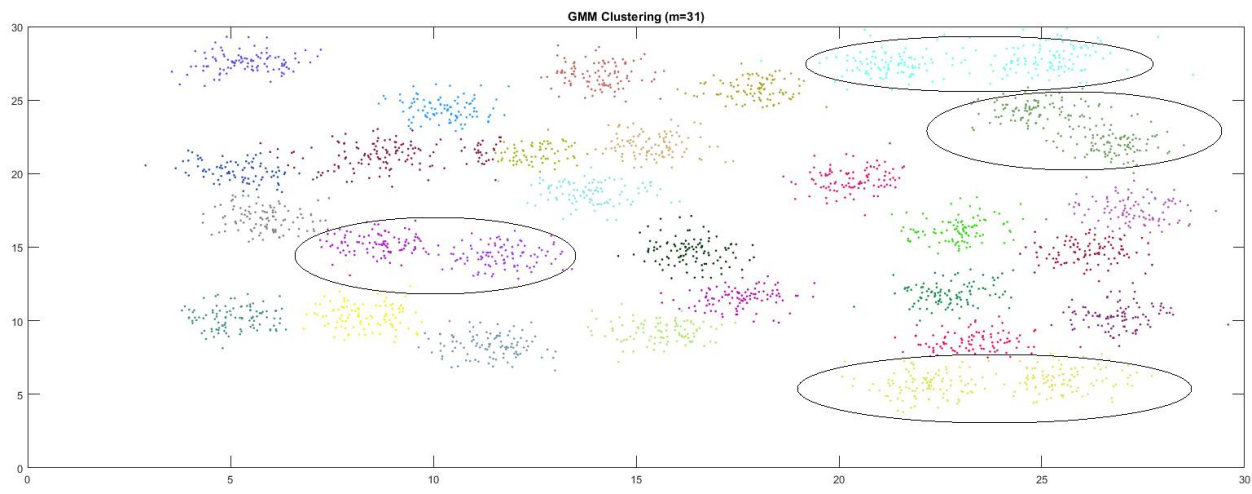

(a) Example GMM clustering solution with $\mathrm{m}=31$

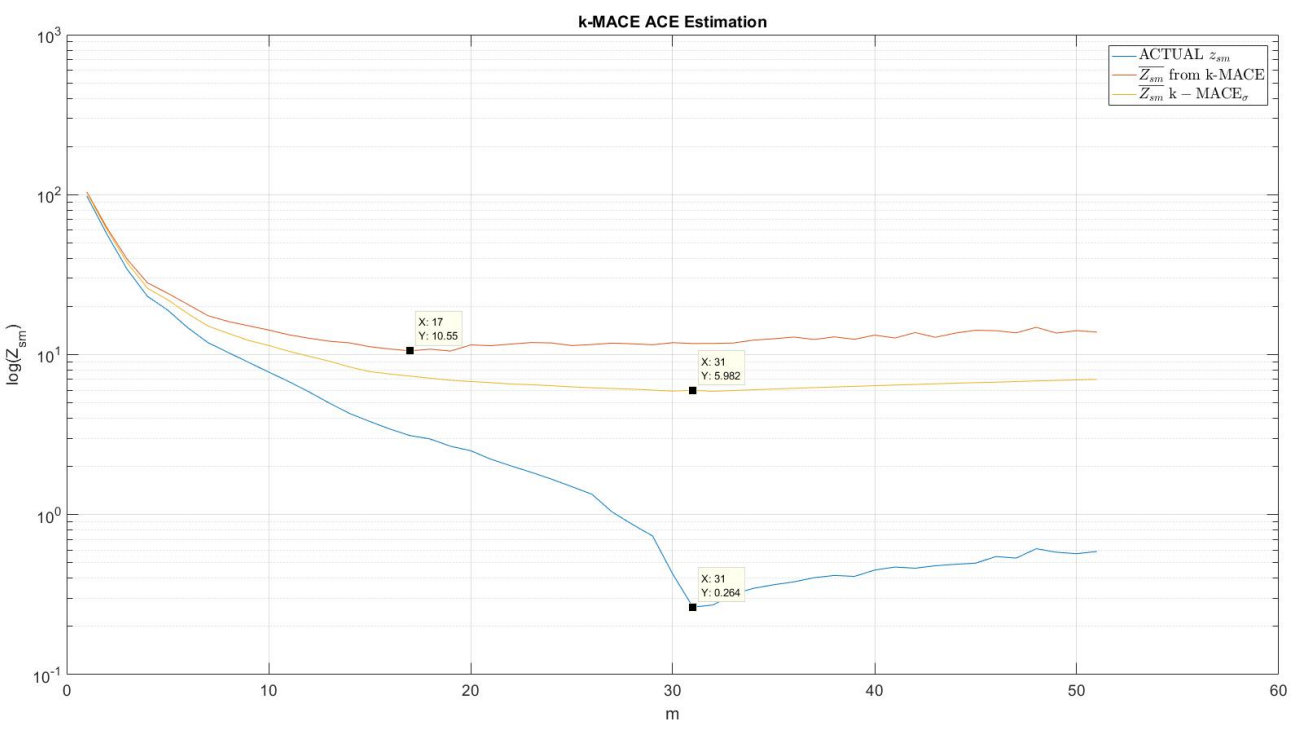

(b) ACE upperbound estimation for D31: $\mathrm{k}-\mathrm{MACE}$ vs $\mathrm{k}-\mathrm{MACE}_{\sigma}$

Figure 4.3: k-MACE on D31 data set

\subsection{Smart k-MACE $\sigma$ Validation}

As it was discussed in the previous section, with the prior assumption that clusters are IID, it is more efficient to use $\mathrm{k}-\mathrm{MACE}_{\sigma}$ over $\mathrm{k}-\mathrm{MACE}$. In practical applications however, such knowledge may not be available. In order to check whether or not data set is composed of IID clusters with uniform variance, we propose to first use $\mathrm{k}-\mathrm{MACE}_{\sigma}$. 
We validate whether the clustering result from $\mathrm{k}-\mathrm{MACE}_{\sigma}$ form clusters that are IID by checking unimodality of all the touching clusters. If two touching clusters that are the output from $\mathrm{k}-\mathrm{MACE}_{\sigma}$ turned out to be unimodal, then the hypothesis that all clusters are IID with the same variance does not apply to the data. Therefore, $\mathrm{k}-\mathrm{MACE}$ must be used instead of $\mathrm{k}-\mathrm{MACE}_{\sigma}$. More information about unimodal test of touching clusters is explained on the following section.

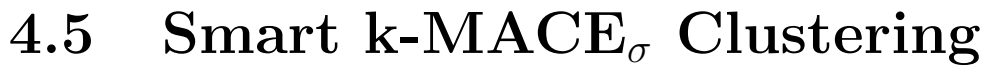

Figure 4.4 shows an example of how $\mathrm{k}-\mathrm{MACE}_{\sigma}$ performs on clusters that has general Gaussian distribution. Assuming that all clusters shares the same variance, $\mathrm{k}-\mathrm{MACE}_{\sigma}$ aims to cluster the data where all the clusters have equal variance. As a result, it ended up splitting clusters that are widely spread into multiple clusters since by doing so, $\mathrm{k}-\mathrm{MACE}_{\sigma}$ ensures that all the clusters that are formed are cohesively similar (i.e. $\left.y_{s_{m 2}} \approx y_{s_{m 2}} \approx, \ldots, y_{s_{m} \hat{m}}\right)$. This ultimately results into $\mathrm{k}-\mathrm{MACE}_{\sigma}$ overestimating the number of clusters.

In this section, we further developed $\mathrm{k}-\mathrm{MACE}_{\sigma}$ to be able to validate the clustered data after the clustering process. The proposed method, denoted by smart $\mathrm{k}-\mathrm{MACE}_{\sigma}$, looks for two touching clusters at time, and determine whether these clusters were appropriately split or not through Chi-square goodness of fit unimodality test. If this pair of touching clusters is indeed a sample of a unimodal distribution, we recombine these clusters. The process of finding and recombining touching clusters is iteratively done until all touching clusters have undergone the test for unimodality. 


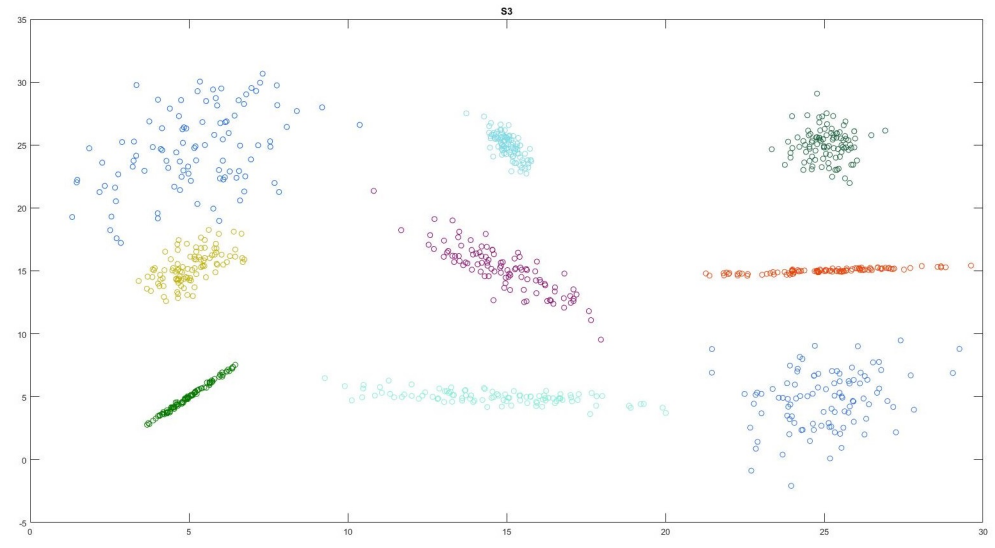

(a) Data Set $(\mathrm{m}=9$, Generally Distributed, unique covariance)

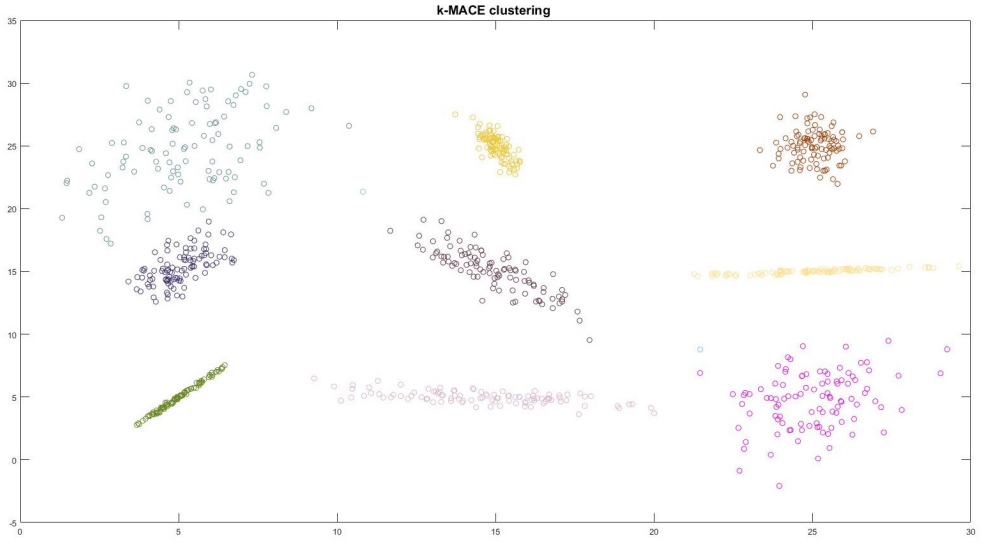

(b) $\mathrm{k}-\mathrm{MACE}$ clustering $(\hat{m}=9)$

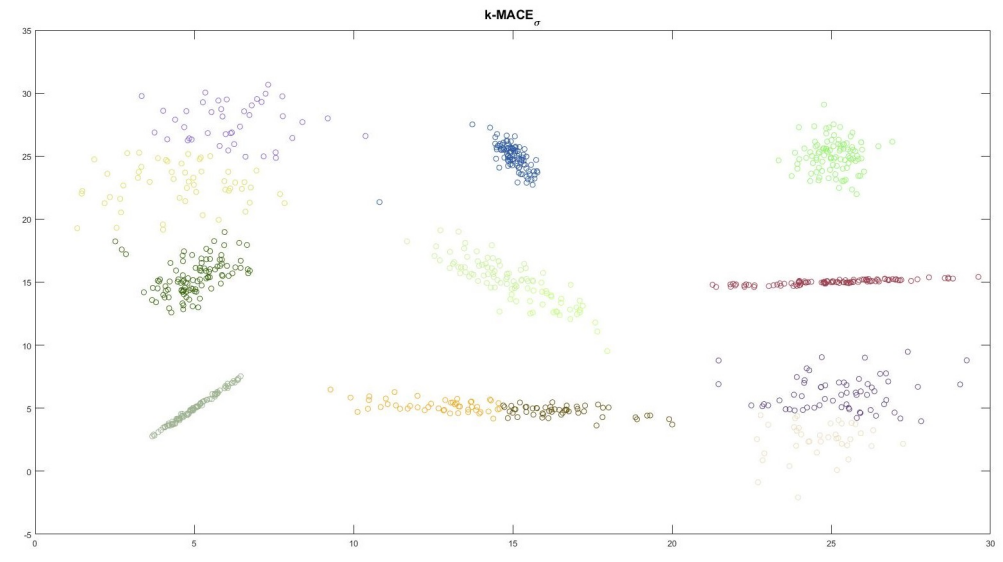

(c) $\mathrm{k}-\mathrm{MACE}_{\sigma}$ clustering $(\hat{m}=13)$

Figure 4.4: Example Clustering Solution: $\mathrm{k}-\mathrm{MACE}$ vs $\mathrm{k}-\mathrm{MACE}_{\sigma}$ on general Gaussian clusters 


\subsubsection{Indentifying Touching Clusters}

From the clustering solution provided by $\mathrm{k}-\mathrm{MACE}_{\sigma}$, we can calculate the overall cluster compactness $y_{s_{\hat{m}}}$ using equations (3.13) and (3.14).

Let us denote $d\left(\hat{C}_{i}, \hat{C}_{j}\right)$ as the distance between clusters $C_{i}$ and $C_{j}$. More specifically, $d\left(\hat{C}_{i}, \hat{C}_{j}\right)$ pertains to the smallest distance between two elements, one coming from cluster $\hat{C}_{i}$ and the other one coming from cluster $\hat{C}_{j}$. In other words, $d\left(\hat{C}_{i}, \hat{C}_{j}\right)$ is the distance between the closest elements from clusters $\hat{C}_{i}$ and $\hat{C}_{j}$. Clusters $\hat{C}_{i}$ and $\hat{C}_{j}$ are considered to be touching when $d^{2}\left(\hat{C}_{i}, \hat{C}_{j}\right) \leq y s m$.

\subsubsection{Unimodality Test for Touching Clusters}

If clusters $\hat{C}_{i}$ and $\hat{C}_{j}$ are touching, we form a data set $x$ by combining these two clusters such that:

$$
x=\left[x_{1}, x_{2}, \ldots, x_{N^{\prime}}\right] \mid x_{k} \in\left(\hat{C}_{i} \cup \hat{C}_{j}\right)
$$

where $N^{\prime}$ is the total number of elements in Cluster $\hat{C}_{i}$ and $\hat{C}_{j}$ combined.

Most of existing statistical test for determining unimodality of data such as KilmogorovSmirnov (KS) and Chi-square goodness of fit is applicable only on one-dimensional data. Therefore, a necessary step is to transform $x$ from a $d$-dimensional data into a onedimensional data.

$$
y=f(x)
$$

where $f$ is transformation of $x$ into one-dimensional data $y$. In our algorithm, Principal Component Analysis (PCA) is used for such transformation. 
The next step for this validation process is to check whether or not $y$ came from a unimodal distribution. But before we do this, we first normalize $y$ so that it has mean of zero and a variance of 1 .

$$
z=\frac{y-\operatorname{mean}(y)}{\operatorname{std}(y)}
$$

The hypothesis being tested is whether or not $z$ are samples that came from normal distribution $\mathcal{N}(0,1)$. Chi-square goodness of fit with significance level of $5 \%$ is employed for this test. If $y$ is indeed samples that came from $\mathcal{N}(0,1)$, then clusters $\hat{C}_{i}$ and $\hat{C}_{j}$ are said to be inappropriately split. Few examples of touching clusters where unimodality test must be applied are depicted on Figures 4.5 - 4.8 .

Chi-square goodness of fit was able to determine that only the case for 4.5 is that the touching clusters should be counted as a single cluster. For the rest of the cases, Chisquare goodness of fit was able to determine that these pairs of clusters is appropriately divided into 2. Smart $\mathrm{k}-\mathrm{MACE}_{\sigma}$ clustering takes the output of the $\mathrm{k}-\mathrm{MACE}_{\sigma}$ and performs the recombination process described.

Figure 4.7 shows the case when two IID clusters with equal variance are touching. In this case, chi-square goodness of fit test determined that these two clusters are not unimodal and therefore, should not be combined. The rest of the touching IID clusters in this data 4.7 failed the unimodality test so there where no touching clusters that were combined. When such case happens, the use of $\mathrm{k}-\mathrm{MACE}_{\sigma}$ over $\mathrm{k}-\mathrm{MACE}$ is validated.

This algorithm for this recombination process is described in Algorithm 3. 


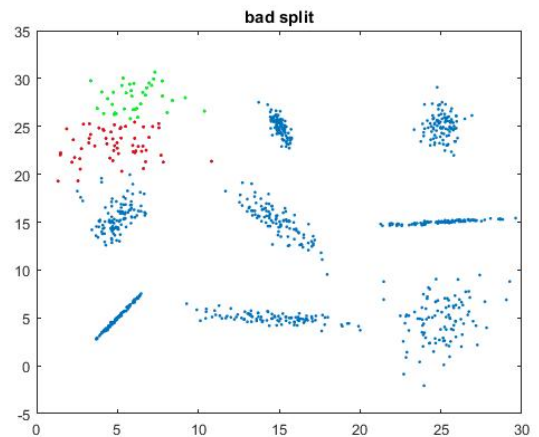

Figure 4.5: Touching clusters formed by splitting of a unimodal cluster

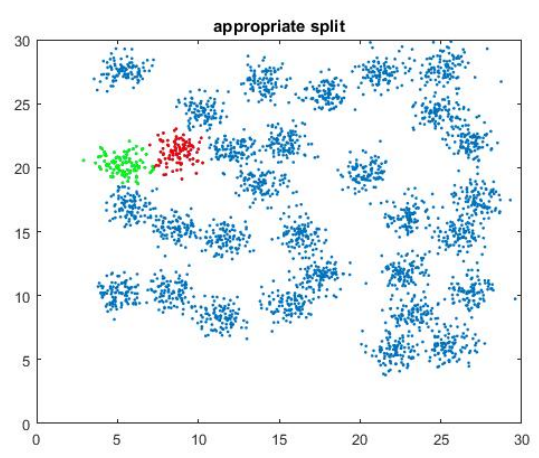

Figure 4.7: Two touching IID clusters

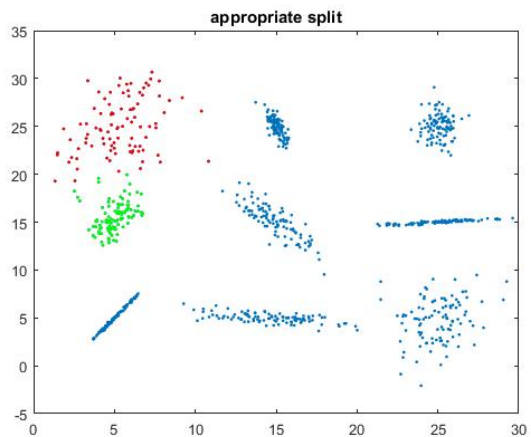

Figure 4.6: Two generally distributed touching clusters

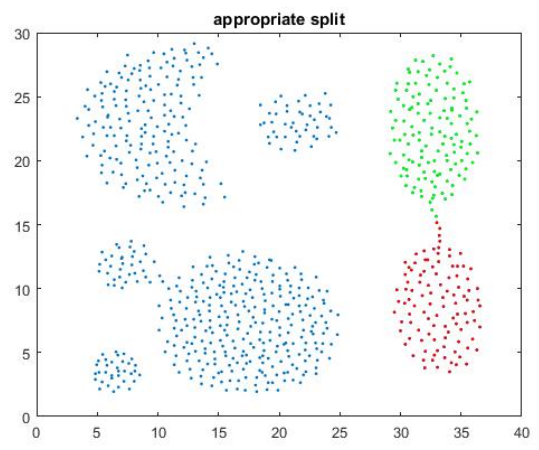

Figure 4.8: Clusters connected through a neck 


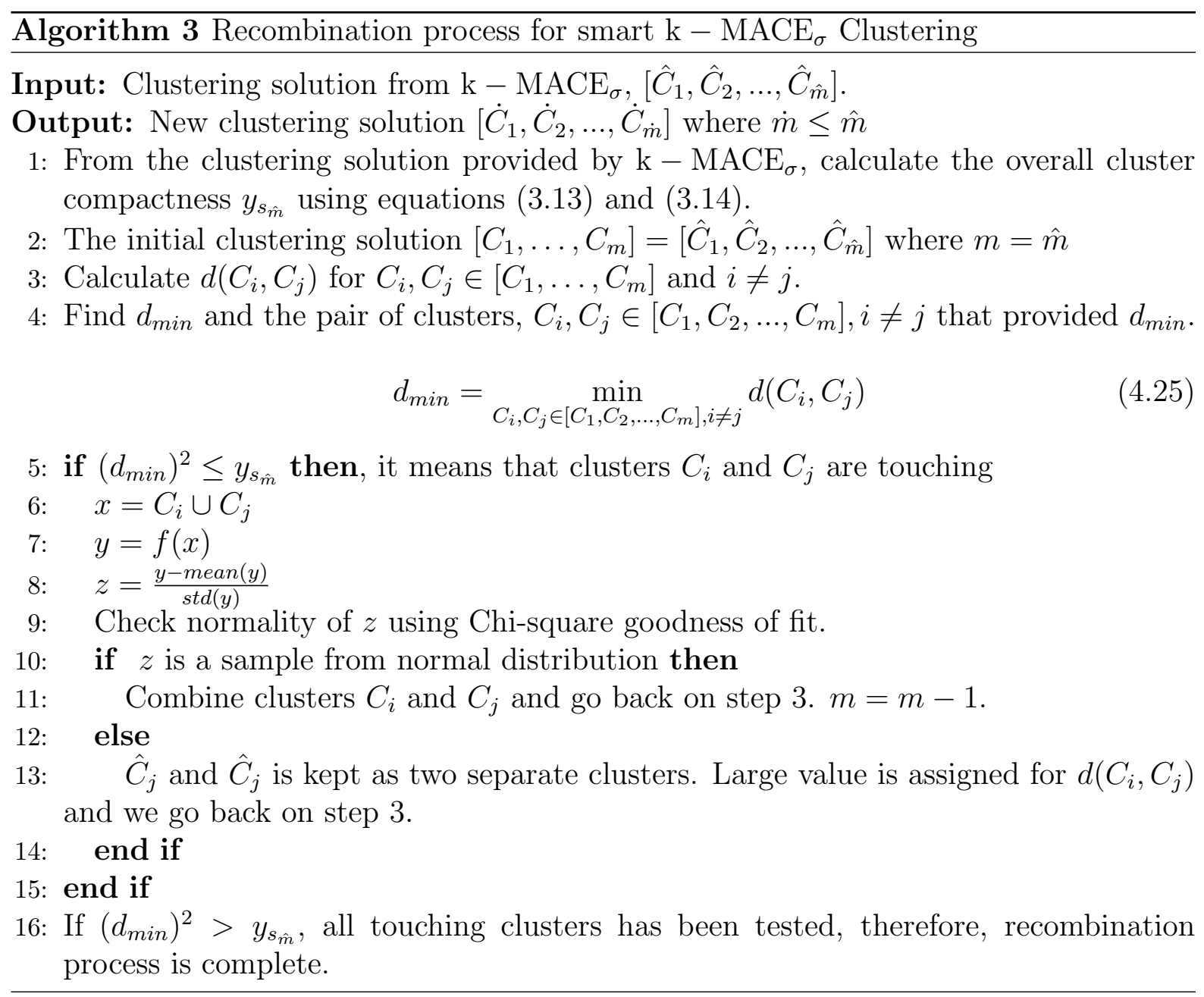





\section{Chapter 5}

\section{Simulations and Result}

\subsection{Artificial Data Sets}

We have generated 4 types of data set depicted on Figures 5.1 5.4 . These data set has varying complexity in terms of degrees of overlap, type of cluster distribution, and number of elements. Each data set is tested both with $\mathrm{k}-\mathrm{MACE}, \mathrm{k}-\mathrm{MACE}_{\sigma}$ and MACE means. Figures 5.1 5.4 shows graphical results that proves the superiority of the $\mathrm{k}$ - MACE algorithm compared to MACE means not only in terms of estimating the $\mathrm{CNC}$ but as well as better estimation of the unobservable average central error.

Figure 5.1 exhibits the result of our algorithm when applied on a data set with IID clusters. Note that ACE are plotted separately due to the discrepancy between how ACE is defined in $\mathrm{k}-\mathrm{MACE}$ and MACE-means. $\hat{m}_{\mathrm{k}-\mathrm{MACE}}, \hat{m}_{\mathrm{k}-\mathrm{MACE}}$, and $\hat{m}_{\mathrm{MM}}$ are the estimated number of cluster using $\mathrm{k}-\mathrm{MACE}, \mathrm{k}-\mathrm{MACE}_{\sigma}$, and MACE-means, respectively. Although MACE-means was able to identify the CNC, our algorithm $\hat{m}_{\mathrm{k}-\mathrm{MACE}}$ and $\hat{m}_{\mathrm{k}-\mathrm{MACE}_{\sigma}}$, provides a more accurate and smoother estimate of the unobservable error. This is because our algorithms takes into account the variance of both the ACE 

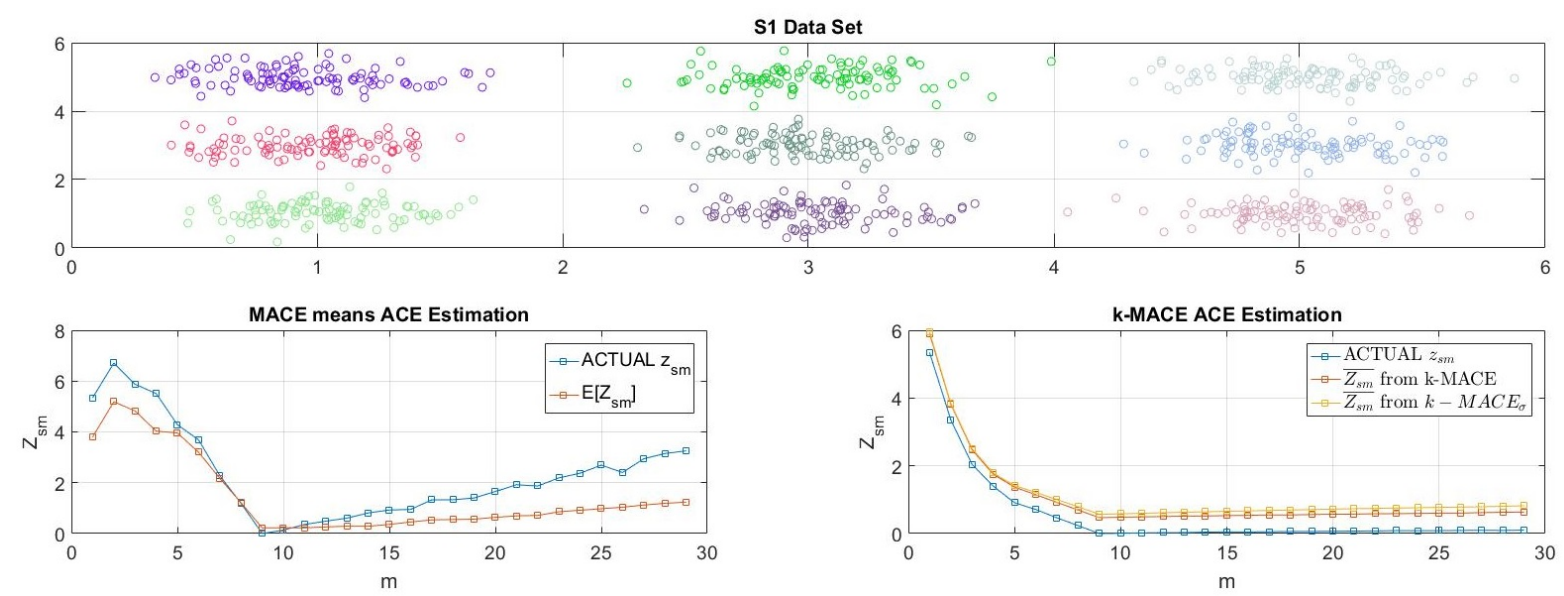

Figure 5.1: IID clusters, uniform variance and uniform proximity (data set characteristic: $\bar{m}=9, d=2, N=900\left(\right.$ result: $\hat{m}_{\mathrm{k}-\mathrm{MACE}}=9, \hat{m}_{\mathrm{k}-\mathrm{MACE}_{\sigma}}=9, \hat{m}_{\mathrm{MM}}=9$ )

and data errors whereas as MACE means only consideres their expected values.

Figure 5.2 is composed of a data set where clusters are still IID, but not sharing the same variance. The actual ACE based on MACE means and k-MACE still reaches its minimum value at $m=5$. Looking at the minimum point of the estimated $\mathrm{ACE}$, what we see is that the $\mathrm{k}-\mathrm{MACE}$ 's CNC estimate is 4 while MACE means and $\mathrm{k}-\mathrm{MACE}_{\sigma}$ over estimated the CNC at $\hat{m}_{M M}=6$ and $\hat{m}_{\mathrm{k}-\mathrm{MACE}_{\sigma}}=9$, respectively. $\mathrm{k}-\mathrm{MACE}_{\sigma}$ overly estimated the CNC this since method inherently clusters the data in a way that all the cluster have the same compactness, as explained in Section 4.2. k- MACE, on the other hand, $\hat{m}_{\mathrm{k}-\mathrm{MACE}}$ does not have such restriction which is why it considered the two overlapping clusters as a single cluster since the combination of these two clusters still forms a Gaussian distribution with a variance larger than the remaining 3 clusters.

For Figure 5.3, results show that MACE means and $\mathrm{k}-\mathrm{MACE}_{\sigma}$ over estimated the CNC while $\mathrm{k}-\mathrm{MACE}$ is able to estimate CNC correctly. As explained from Section 4.2, both MACE-means and $\mathrm{k}-\mathrm{MACE}_{\sigma}$ are formulated based on the assumption that clusters are IID and has the same variance. As a result, these two algorithms tends to 

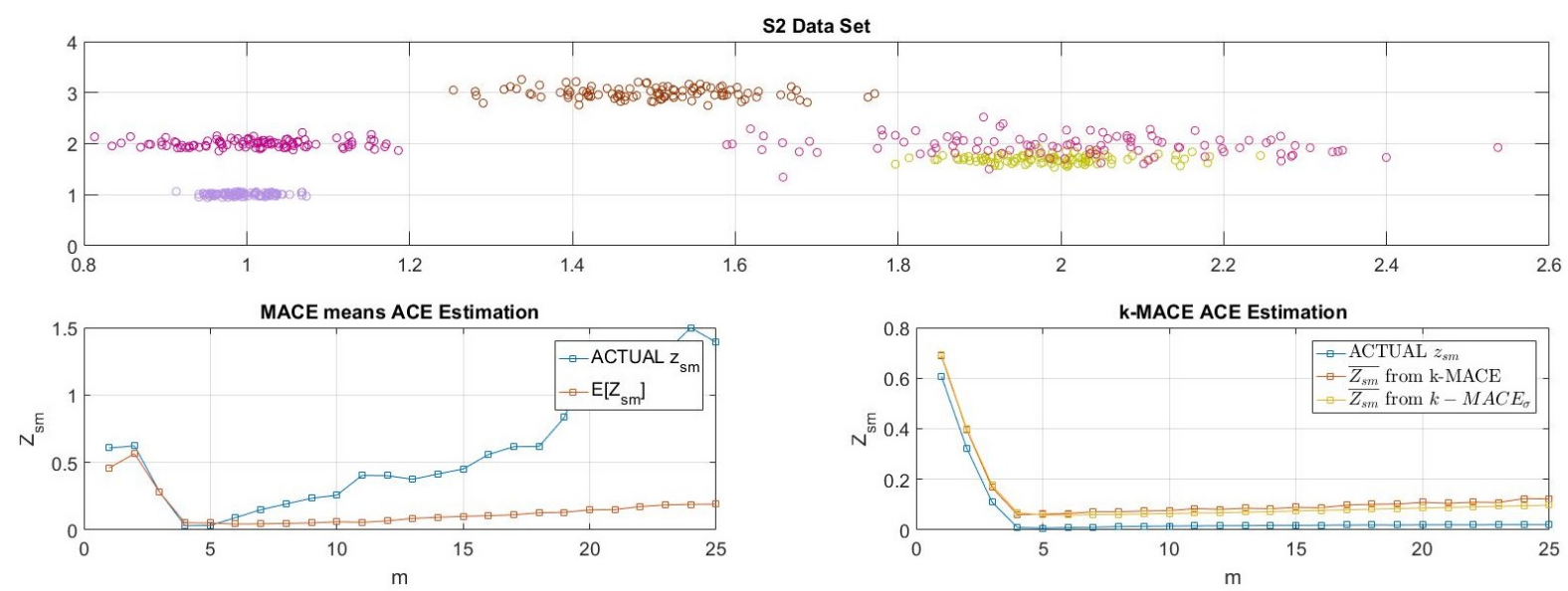

Figure 5.2: IID clusters, unique variance, unique proximity (data set characteristic: $\bar{m}=$ $5, d=2, N=1731)\left(\right.$ result: $\left.\hat{m}_{\mathrm{k}-\mathrm{MACE}}=4, \hat{m}_{\mathrm{k}-\mathrm{MACE}_{\sigma}}=9, \hat{m}_{\mathrm{mm}}=6\right)$
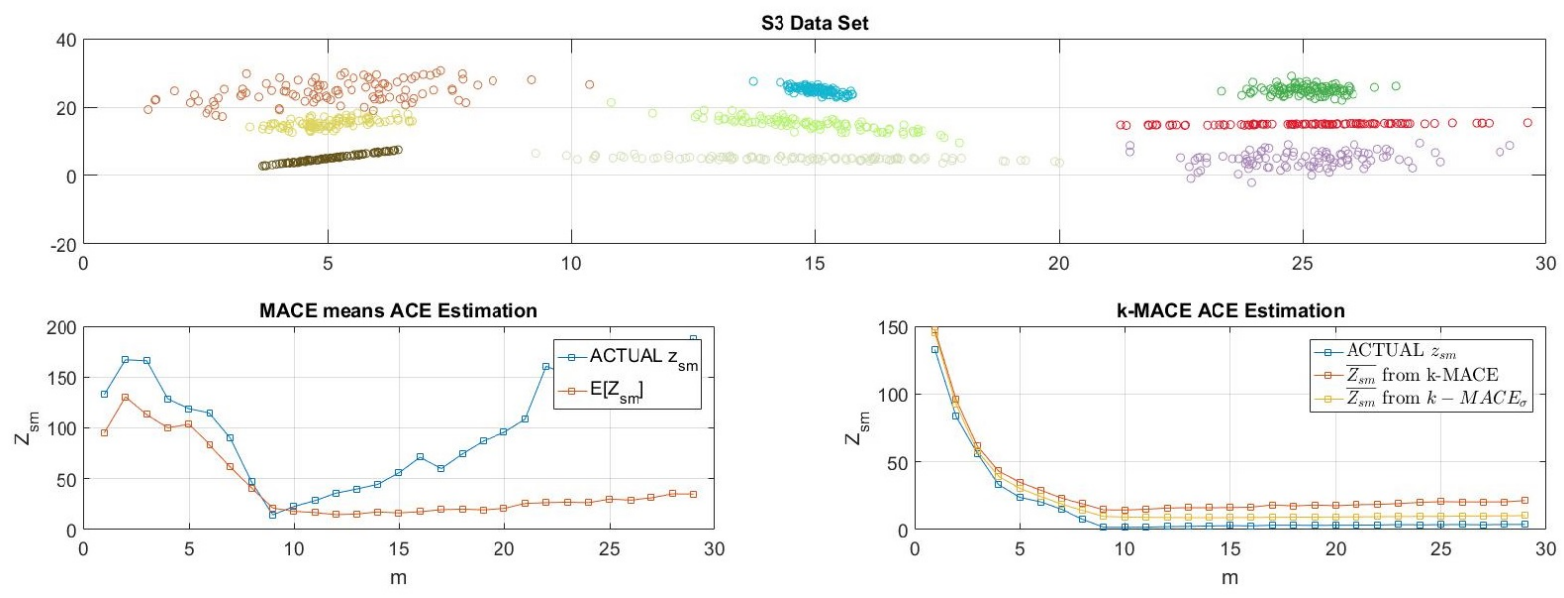

Figure 5.3: General Gaussian, unique covariance, uniform proximity (data set characteristic: $\bar{m}=9, d=2, N=900)\left(\right.$ result: $\hat{m}_{\mathrm{k}-\mathrm{MACE}}=9, \hat{m}_{\mathrm{k}-\mathrm{MACE}_{\sigma}}=14, \hat{m}_{\mathrm{mm}}=13$ ) 

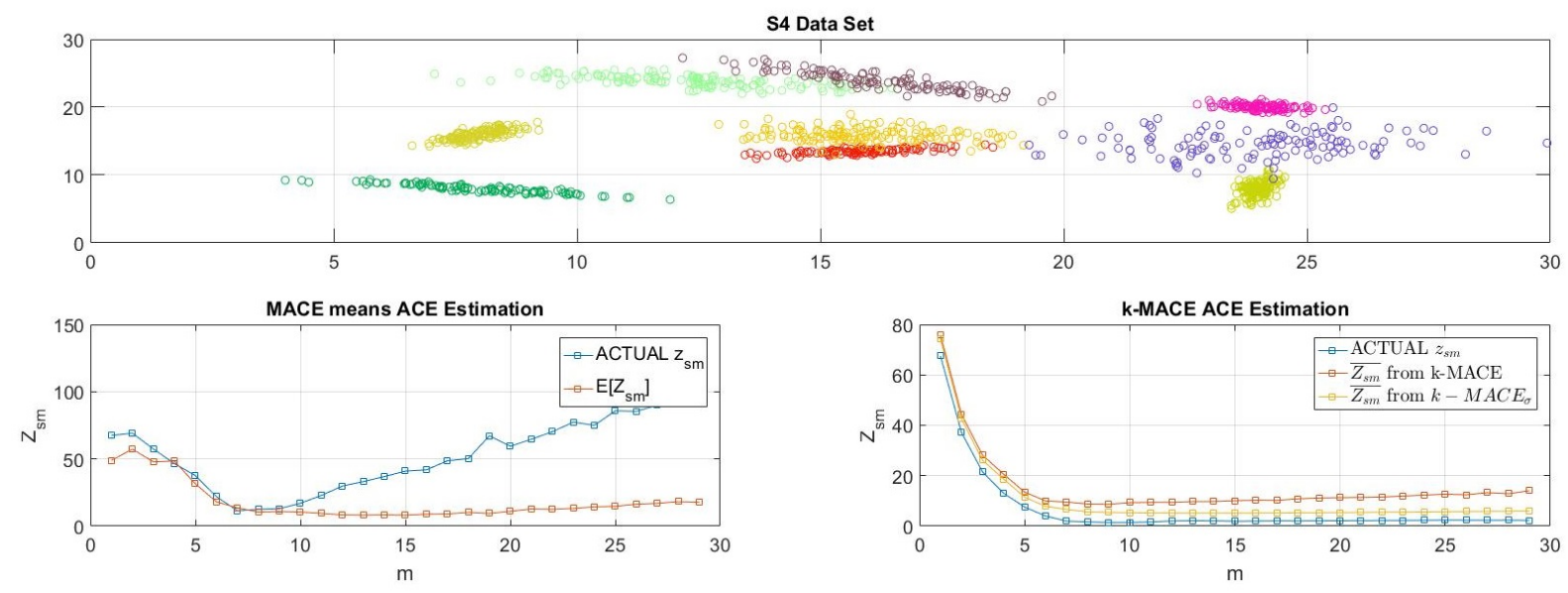

Figure 5.4: General Gaussian, Unique covariance, varying proximity (data set characteristic: $\bar{m}=9, d=2, N=900$ ) (result: $\hat{m}_{\mathrm{k}-\mathrm{MACE}}=9, \hat{m}_{\mathrm{k}-\mathrm{MACE}_{\sigma}}=13, \hat{m}_{\mathrm{mm}}=15$ )

split the data set in a way that all clusters have the same cohesiveness which ultimately results into overestimation of $\mathrm{CNC}$. On the other hand, $\mathrm{k}-\mathrm{MACE}$ formulation assumes that clusters are Gaussian with any covariance structure which is why for data set such as $\mathrm{s} 3, \mathrm{k}-\mathrm{MACE}$ is successful on determining the CNC.

In Figure 5.4, we created a data set that contains clusters that has varying covariance structure and clusters have varying degrees of overlap. Both $\mathrm{k}-\mathrm{MACE}_{\sigma}$ and MACEmeans overestimated the CNC while $\mathrm{k}$ - MACE was able to identify the CNC correctly. The potential of $\mathrm{k}-\mathrm{MACE}$ is showcased in this data set. As can be seen from Table 5.1. amongst the algorithm that we compared $\mathrm{k}-\mathrm{MACE}$ to, $\mathrm{k}-\mathrm{MACE}$ provides most accurate answer with comparable robustness for the s4 data set.

In addition to our own synthetic data set, we also tested our algorithm to some of synthetic data set used in literatures [29], 30]. Result are shown on Figures 5.5,5.7.

Table 5.1 provides a comparison of result for synthetic data set using some of known methods for classifying correct number of clusters. 


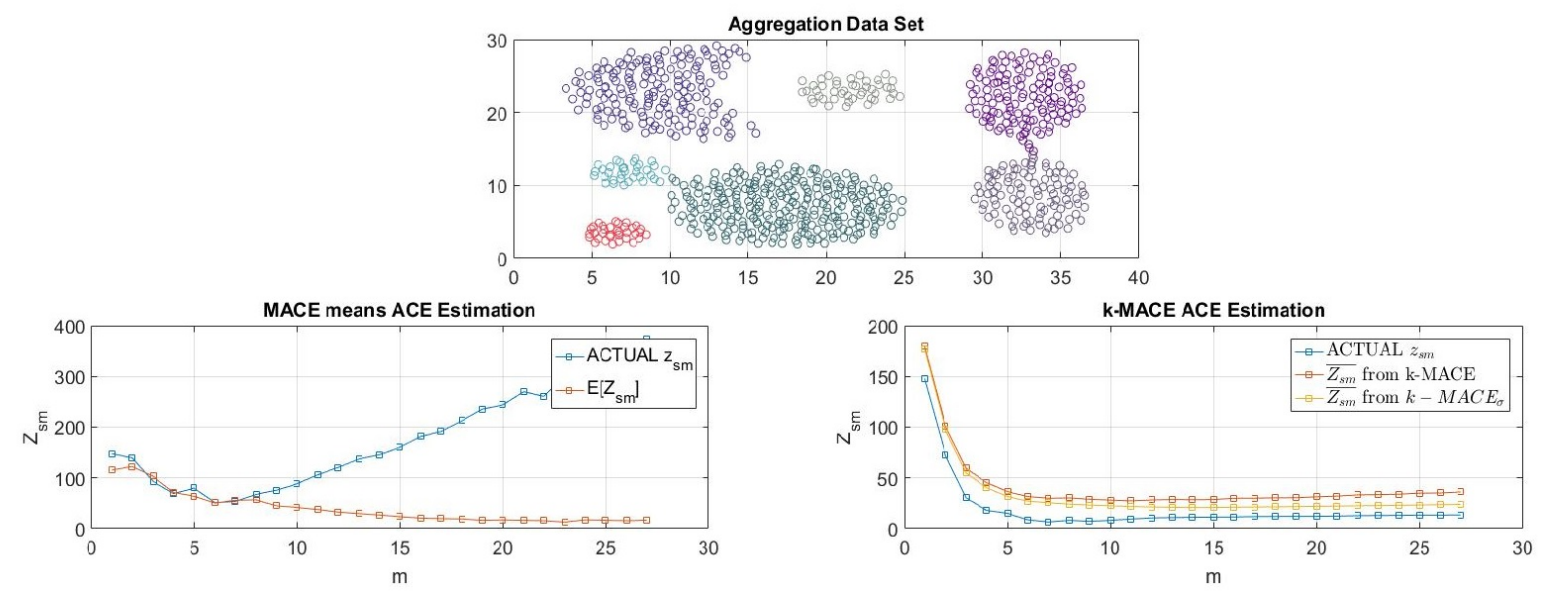

Figure 5.5: Aggregation data set (data set characteristic: $\bar{m}=7, d=2, N=787$ ) (result: $\left.\hat{m}_{\mathrm{k}-\mathrm{MACE}}=11, \hat{m}_{\mathrm{k}-\mathrm{MACE}_{\sigma}}=14, \hat{m}_{\mathrm{mm}}=23\right)$

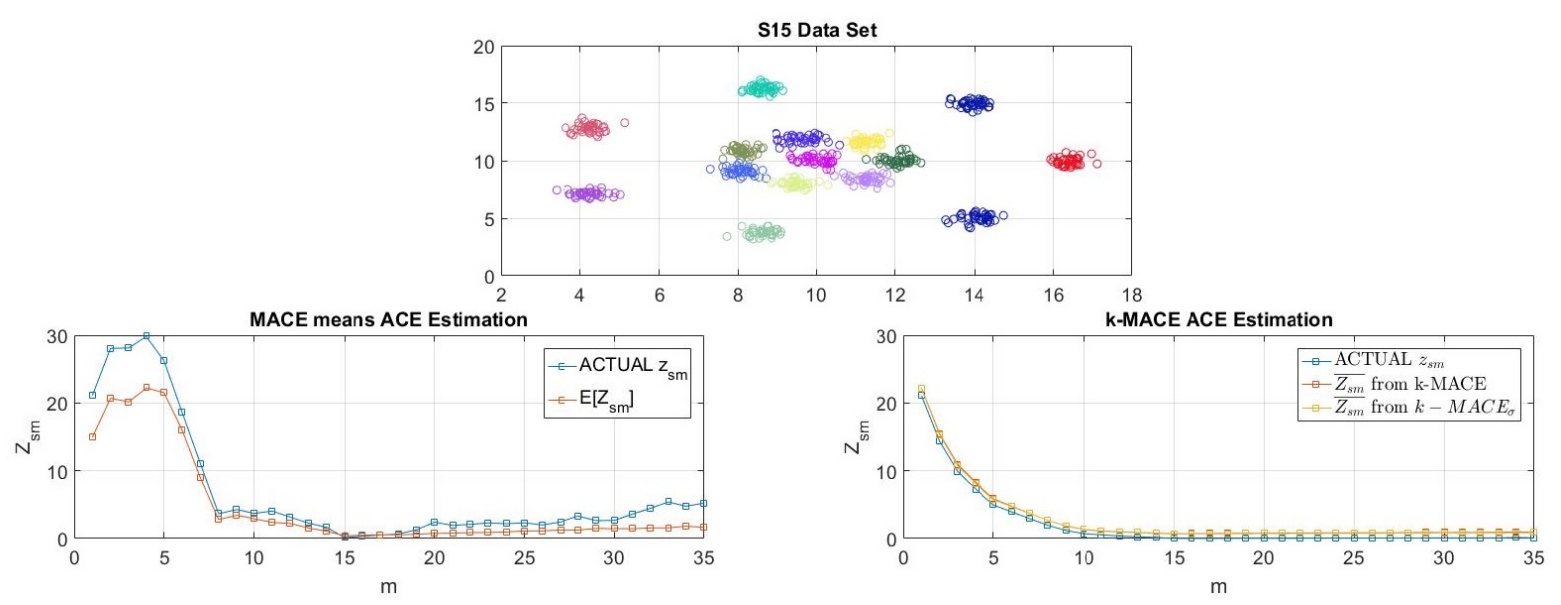

Figure 5.6: R15 data set (data set characteristic: $\bar{m}=15, d=2, N=600$ ) (result: $\left.\hat{m}_{\mathrm{k}-\mathrm{MACE}}=15, \hat{m}_{\mathrm{k}-\mathrm{MACE}_{\sigma}}=15, \hat{m}_{\mathrm{mm}}=15\right)$ 


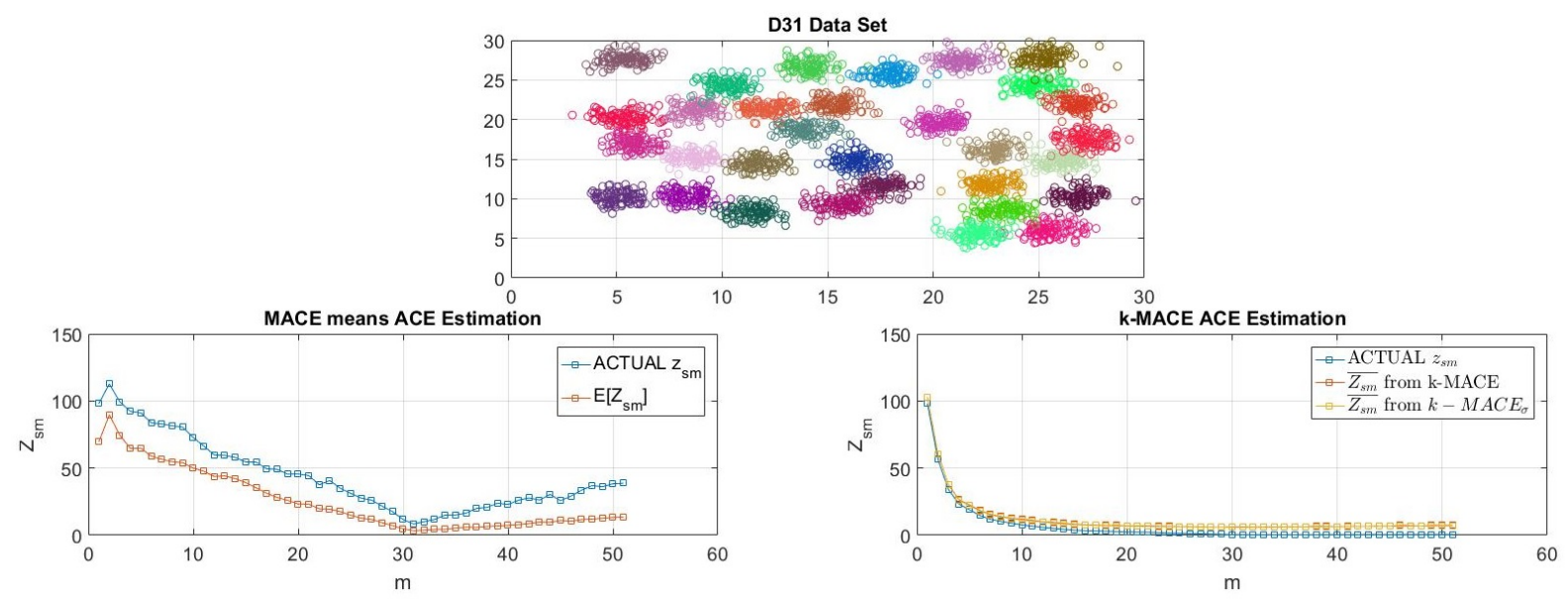

Figure 5.7: D31 data set (data set characteristic: $\bar{m}=31, d=2, N=3100$ ) (result: $\left.\hat{m}_{\mathrm{k}-\mathrm{MACE}}=22, \hat{m}_{\mathrm{k}-\mathrm{MACE}_{\sigma}}=31, \hat{m}_{\mathrm{mm}}=31\right)$ 
Table 5.1: Artificial data set. Result is generated from the average of 50 runs. Results are in the form of $E[\hat{m}] \pm s t d[\hat{m}]$

\begin{tabular}{|l|l|l|l|l|l|l|l|}
\hline & $\begin{array}{l}\mathrm{S} 1 \\
\mathrm{~m}=9\end{array}$ & $\begin{array}{l}\mathrm{S} 2 \\
\mathrm{~m}=5\end{array}$ & $\begin{array}{l}\mathrm{S} 3 \\
\mathrm{~m}=9\end{array}$ & $\begin{array}{l}\mathrm{S} 4 \\
\mathrm{~m}=9\end{array}$ & $\begin{array}{l}\text { Aggregation } \\
\mathrm{m}=7\end{array}$ & $\begin{array}{l}\mathrm{R} 15 \\
\mathrm{~m}=15\end{array}$ & $\begin{array}{l}\mathrm{D} 31 \\
\mathrm{~m}=31\end{array}$ \\
\hline $\mathrm{k}-M A C E^{\mathbf{9} \pm \mathbf{0}}$ & $\mathbf{5 . 1} \pm \mathbf{0 . 3}$ & $\mathbf{9} \pm \mathbf{0}$ & $\mathbf{8 . 7} \pm \mathbf{0 . 2}$ & $8.9 \pm 0.5$ & $\mathbf{1 5} \pm \mathbf{0}$ & $19.7 \pm 0.9$ \\
\hline $\mathrm{k}-\mathrm{MACE}_{\sigma}$ & $\mathbf{9} \pm \mathbf{0}$ & $\mathbf{5 . 1} \pm \mathbf{0 . 2}$ & $12.7 \pm 0.5$ & $11.4 \pm 0.8$ & $14 \pm 1.2$ & $\mathbf{1 5} \pm \mathbf{0}$ & $\mathbf{3 1} \pm \mathbf{0}$ \\
\hline Smart k $-\mathrm{MACE}_{\sigma}$ & $\mathbf{9} \pm \mathbf{0}$ & $4 \pm 0$ & $\mathbf{9} \pm \mathbf{0}$ & $8 \pm 0$ & $\mathbf{7} \pm \mathbf{0}$ & $\mathbf{1 5} \pm \mathbf{0}$ & $\mathbf{3 1} \pm \mathbf{0}$ \\
\hline MACE-means & $\mathbf{9} \pm \mathbf{0}$ & $7 \pm 0.8$ & $12.1 \pm 0.3$ & $14 \pm 0$ & $16 \pm 0.6$ & $\mathbf{1 5} \pm \mathbf{0}$ & $31.8 \pm 0.6$ \\
\hline Dipmeans & $\mathbf{9} \pm \mathbf{0}$ & $4 \pm 0$ & $\mathbf{9} \pm \mathbf{0}$ & $7 \pm 0$ & $5 \pm 0$ & $8 \pm 0$ & $23 \pm 0$ \\
\hline Gmeans & $\mathbf{9} \pm \mathbf{0}$ & $6 \pm 0$ & $11 \pm 0$ & $17 \pm 0$ & $9 \pm 0$ & $16 \pm 0$ & $31 \pm 0$ \\
\hline DBSCAN & $\mathbf{9} \pm \mathbf{0}$ & $2 \pm 0$ & $11 \pm 0$ & $11 \pm 0$ & $1.0 \pm 0.0$ & $\mathbf{1 5} \pm \mathbf{0}$ & $34.0 \pm 0.0$ \\
\hline $\mathrm{CH}+$ K-means & $\mathbf{9} \pm \mathbf{0}$ & $4.9 \pm 0.25$ & $17 \pm 0$ & $17.4 \pm 1.30$ & $15.7 \pm 0.9$ & $15.3 \pm 0.5$ & $33.4 \pm 1.1$ \\
\hline Sil + K-means & $\mathbf{9} \pm \mathbf{0}$ & $4 \pm 0$ & $\mathbf{9} \pm \mathbf{0}$ & $7.8 \pm 0.4$ & $3.0 \pm 0.0$ & $15.3 \pm 0.5$ & $30.9 \pm 1.5$ \\
\hline DB + K-means & $\mathbf{9} \pm \mathbf{0}$ & $4 \pm 0$ & $\mathbf{9} \pm \mathbf{0}$ & $7.7 \pm 0.5$ & $4.0 \pm 0.0$ & $12.2 \pm 3.5$ & $28.4 \pm 2.0$ \\
\hline Xie-Beni + K-means & $\mathbf{9} \pm \mathbf{0}$ & $4 \pm 0$ & $\mathbf{9} \pm \mathbf{0}$ & $6 \pm 0.5$ & $4.0 \pm 0.0$ & $8 \pm 3.5$ & $30.2 \pm 0.7$ \\
\hline Dunn + K-means & $\mathbf{9} \pm \mathbf{0}$ & $4 \pm 0$ & $\mathbf{9} \pm \mathbf{0}$ & $4 \pm 0.7$ & $16.2 \pm 1.0$ & $12.2 \pm 3.5$ & $34 \pm 4.9$ \\
\hline
\end{tabular}


From Table 5.1, most algorithm provides accurate result when dealing with data set that is composed of clusters that are IID and has the same variance as signified by the result from s1 and R15.

Looking at performance of $\mathrm{k}-\mathrm{MACE}$ from Table 5.1, it can be noticed that $\mathrm{k}-\mathrm{MACE}$ provides the most accurate estimate of $\mathrm{CNC}$ for data set s3 and s4 where the clusters have general Gaussian distribution. Moreover, for these synthetic data set, the standard deviation of CNC estimate, std $[\hat{m}]$, of $\mathrm{k}-\mathrm{MACE}$ has a low value. This small standard deviation signifies robustness of our method as it was able to provide consistent results even when dealing with Gaussian clusters that are touching which is the case for data set s4. Overall, when data set is composed of Gaussian cluster, k- MACE outperforms the competing methods in terms of accuracy and precision. $\mathrm{k}$ - MACE also performed well on data sets S1 and R15 where clusters are IID. For these data set, the clusters are well separated and so k-MACE doesn't suffer from bad estimation of cluster covariance as discussed on chapter 4.2 .

Meanwhile,the following observations for $\mathrm{k}-\mathrm{MACE}_{\sigma}$ and Smart $\mathrm{k}-\mathrm{MACE}_{\sigma}$ from Table 5.1 are discussed below:

For data set S3, where the clusters are general Gaussian distributed, stand-alone $\mathrm{k}-\mathrm{MACE}_{\sigma}$ overestimate the CNC. The recombination step for Smart k- MACE $\mathrm{F}_{\sigma}$ was able to fix such errors as the CNC was correctly reduced to 9. Note that in Smart $\mathrm{k}-\mathrm{MACE}_{\sigma}$, the recombination step doesn't require calculation of the ACE's upperbound estimate which is why the plot for ACE is not shown for this method.

For data set D31, where the clusters are IID and clusters have varying degrees of overlap, $\mathrm{k}-\mathrm{MACE}_{\sigma}$ provides the most accurate and consistent estimate of CNC. Moreover, $\mathrm{k}-\mathrm{MACE}_{\sigma}$ outperforms MACE means although these two algorithms are based on the same assumption that data set is composed of IID clusters. MACE means ignored the 
variance of $\mathrm{ACE}$ and only estimates $\mathrm{ACE}$ 's expected value. $\mathrm{k}-\mathrm{MACE}_{\sigma}$ on the other hand considered the variances of both $\mathrm{ACE}$ and data error, thus providing a more robust and accurate estimate of the ACE. As for Smart k- $\mathrm{MACE}_{\sigma}$, note that $\mathrm{CNC}$ estimate is also 31 which means that no touching clusters are futher combined which shows the effectiveness of Chi-square goodness of fit as our unimodal test for touching clusters. The fact that no clusters are further are recombined also validates that the data set is composed of IID clusters as explained in section 4.3.

For aggregation data set, only Smart $\mathrm{k}-\mathrm{MACE}_{\sigma}$ were able to estimate the $\mathrm{CNC}$ correctly. At first, $\mathrm{k}-\mathrm{MACE}_{\sigma}$ overestimated the $\mathrm{CNC}$ but due to the recombination step of $\mathrm{k}-\mathrm{MACE}_{\sigma}$, touching clusters that are inappropriately divided are recombined. Overall, the result proves that the recombination step on Smart k- MACE $\mathrm{F}_{\sigma}$ were able to alleviate the weakness of $\mathrm{k}-\mathrm{MACE}_{\sigma}$ (it cannot handle General Gaussian Clusters).

\subsection{Real World Bench Mark Data Sets}

Our algorithm is tested on 12 real-world data sets from UCI machine learning repository website 1 seeds, iris, vertebral, wine, abalone, breast, yeast, thyroid, Water Treatment Plant (WTP), glass, soybean and Wisconsin Diagnostic Breast Cancer (WDBC), We have compared our result with some of well known index validity methods such as Calinkski Harabaz, Davis-Bouldin, Silhouette, gap, Dunn and Xie-Beni index. Similar to this validity indexes method, $\mathrm{k}$ - MACE also aims on optimization of an objective function which is the average central error. In addition to this, we also compared it to G-means and DIP-means, since similar to $\mathrm{k}-\mathrm{MACE}$, these two algorithms claims to deal well with clusters that has general Gaussian distribution. We also compared our method with DBSCAN which is one of the most popular clustering algorithm that estimates the

\footnotetext{
${ }^{1}$ http://archive.ics.uci.edu/ml/
} 
number of clusters.

For dataset that are labeled, we have calculated the Adjusted Random Index (ARI) and the Normalized Variation Index (NVI) to measure the similarity between the clustering result and the actual clustering partition. ARI and NVI reflects the similarity between two data clustering. ARI scores that are close to 1 means that the clustering result is close to the actual cluster partition. NVI is the opposite as having a score of 0 signifies a correct clustering result.

The result of this part of the experiment is tabulated on Table 5.2, 5.4. The characteristic of the data set used is also described on these tables where $\bar{m}$ is the actual number of clusters, $d$ is the dimension of the data and $N$ is the number of samples in the data set. The result that gives accurate estimate of CNC is written in bold letters.

$\mathrm{k}$ - MACE gave an accurate and consistent estimate of CNC for seeds, iris, wine, breast and WDBC. Also, having $s t d[\hat{m}]=0$ implies that over 50 runs, $\mathrm{k}-$ MACE consistently estimated the CNC correctly. The ARI and NVI values for $\mathrm{k}-\mathrm{MACE}$ is also one of the most optimal amongst other algorithms we compared $\mathrm{k}-\mathrm{MACE}$ against. Out of all algorithms, k-MACE comes on top as it has the most number of correct estimate of CNC.

An intriguing case is from abalone data set where $\mathrm{k}-\mathrm{MACE}$ severely underestimated the number of cluster. For this particular the data set, $\mathrm{k}-\mathrm{MACE}_{\sigma}$ provided the closest CNC estimate. Applying PCA to abalone data set and taking its first 3 major components, the dimension-reduced data looks as follows.

The 29 clusters in abalone data set is signified by color of dots. Upon inspection, it can be seen that these 29 clusters are inseparable. More importantly, there are 3 main segments on the feature space where samples of abalone data set are heavily dense. Perhaps, when more components are taken into account (dimension is increased in feature 


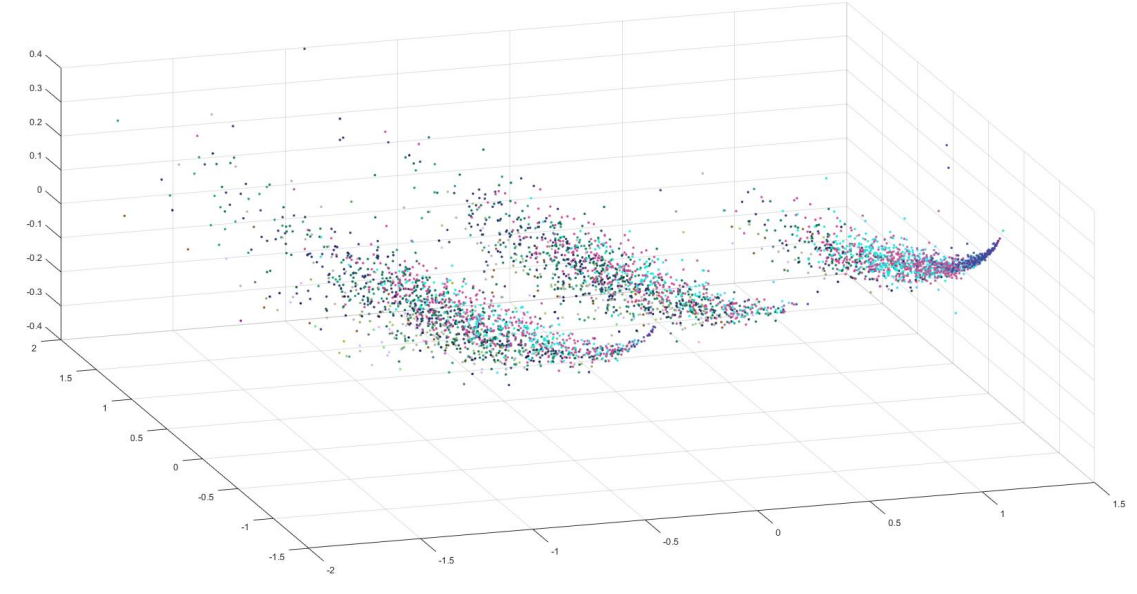

Figure 5.8: Dimension reduced abalone data set

space), these actual clusters will start to become separable and will show more dense areas instead of having only 3. Nonetheless, these 3 main dense areas shown on Figure 5.8 help justify the underestimated CNC, not only for k-MACE but for other methods as well.

For the rest of real life data set, $\mathrm{k}-\mathrm{MACE}, \mathrm{k}-\mathrm{MACE}_{\sigma}$ and smart $\mathrm{k}-\mathrm{MACE}_{\sigma}$. provided comparable results to other existing methods. Taking the average performance of all the clustering algorithm to all real life data set, $\mathrm{k}$ - MACE shows superiority. 
Table 5.2: Real data set 1. Result is generated from the average of 50 runs.

\begin{tabular}{|c|c|c|c|c|c|}
\hline & & $\begin{array}{c}\text { Seeds } \\
\bar{m}=3, \mathrm{~d}=7, \mathrm{~N}=210\end{array}$ & $\begin{array}{c}\text { Iris } \\
\bar{m}=3, \mathrm{~d}=4, \mathrm{~N}=150\end{array}$ & $\begin{array}{c}\text { Vertebral } \\
\bar{m}=3, \mathrm{~d}=6, \mathrm{~N}=310\end{array}$ & $\begin{array}{c}\text { Wine } \\
\bar{m}=3, \mathrm{~d}=13, \mathrm{~N}=178\end{array}$ \\
\hline \multirow{3}{*}{$\mathrm{k}-\mathrm{MACE}$} & $E[m] \pm s t d[m]$ & $\mathbf{3} \pm \mathbf{0}$ & $\mathbf{3} \pm \mathbf{0}$ & $1.8 \pm 0.4$ & $\mathbf{3} \pm \mathbf{0}$ \\
\hline & ARI & 0.7 & 0.7 & 0.3 & 0.5 \\
\hline & NVI & 0.5 & 0.4 & 0.8 & 0.6 \\
\hline \multirow{3}{*}{$\mathrm{k}-\mathrm{MACE}_{\sigma}$} & $E[m] \pm s t d[m]$ & $10.4 \pm 2.5$ & $8 \pm 0$ & $4 \pm 0$ & $6.7 \pm 0.5$ \\
\hline & ARI & 0.3 & 0.5 & 0.3 & 0.5 \\
\hline & NVI & 0.6 & 0.5 & 0.7 & 0.6 \\
\hline \multirow{3}{*}{ Smart k - $\mathrm{MACE}_{\sigma}$} & $E[m] \pm s t d[m]$ & $2.9 \pm 0.3$ & $2 \pm 0$ & $3.0 \pm 0.2$ & $7.3 \pm 0.4$ \\
\hline & ARI & 0.7 & 0.6 & 0.5 & 0.2 \\
\hline & NVI & 0.5 & 0.4 & 0.6 & 0.8 \\
\hline \multirow{3}{*}{ MACE-means } & $E[m] \pm s t d[m]$ & $\mathbf{3} \pm \mathbf{0}$ & $6.5 \pm 0.5$ & $1 \pm 0$ & $11.2 \pm 0$ \\
\hline & ARI & 0.7 & 0.6 & 0 & 0.1 \\
\hline & NVI & 0.5 & 0.5 & 1 & 0.8 \\
\hline \multirow{3}{*}{ DIP-means } & $E[m] \pm s t d[m]$ & $1 \pm 0$ & $2 \pm 0$ & $1 \pm 0$ & $1 \pm 0$ \\
\hline & ARI & 0 & 0.5 & 0 & 0 \\
\hline & NVI & 1 & 0.6 & 1 & 1 \\
\hline \multirow{3}{*}{ G-means } & $E[m] \pm s t d[m]$ & $4 \pm 0$ & $4 \pm 0$ & $5 \pm 0$ & $2 \pm 0$ \\
\hline & ARI & 0.3 & 0.5 & 0.3 & 0.2 \\
\hline & NVI & 0.7 & 0.6 & 0.8 & 0.7 \\
\hline \multirow{3}{*}{ X-means } & $E[m] \pm s t d[m]$ & $45 \pm 0$ & $12 \pm 0$ & $6 \pm 0$ & $40 \pm 0.0$ \\
\hline & ARI & 0.1 & 0.1 & 0.2 & 0.1 \\
\hline & NVI & 0.9 & 0.9 & 0.7 & 0.9 \\
\hline \multirow{3}{*}{ DBSCAN } & $E[m] \pm s t d[m]$ & $2 \pm 0$ & $\mathbf{3} \pm \mathbf{0}$ & $1 \pm 0$ & $1.0 \pm 0.0$ \\
\hline & ARI & 0 & 0.5 & 0 & 0 \\
\hline & NVI & 1 & 0.6 & 1 & 1 \\
\hline \multirow{3}{*}{$\mathrm{CH}+\mathrm{K}$-means } & $E[m] \pm s t d[m]$ & $\mathbf{3} \pm \mathbf{0}$ & $\mathbf{3} \pm \mathbf{0}$ & $4 \pm 0$ & $12.7 \pm 0.5$ \\
\hline & ARI & 0.7 & 0.7 & 0.3 & 0.1 \\
\hline & NVI & 0.5 & 0.4 & 0.7 & 0.8 \\
\hline \multirow{3}{*}{ Sil + K-means } & $E[m] \pm s t d[m]$ & $2 \pm 0$ & $2 \pm 0$ & $3 \pm 0.2$ & $2.0 \pm 0.0$ \\
\hline & ARI & 0.5 & 0.5 & 0.3 & 0.4 \\
\hline & NVI & 0.6 & 0.5 & 0.7 & 0.7 \\
\hline \multirow{3}{*}{ DB + K-means } & $E[m] \pm s t d[m]$ & $2 \pm 0$ & $2 \pm 0$ & $3.1 \pm 0.3$ & $7 \pm 0.5$ \\
\hline & ARI & 0.5 & 0.5 & 0.3 & 0.2 \\
\hline & NVI & 0.6 & 0.5 & 0.7 & 0.8 \\
\hline \multirow{3}{*}{ gap + K-means } & $E[m] \pm s t d[m]$ & $2 \pm 0$ & $2 \pm 0$ & $5.2 \pm 0.5$ & $8.7 \pm 5.2$ \\
\hline & ARI & 0.7 & 0.5 & 0.3 & 0.1 \\
\hline & NVI & 0.5 & 0.5 & 0.7 & 0.9 \\
\hline \multirow{3}{*}{ Xie-Beni + K-means } & $E[m] \pm s t d[m]$ & $2 \pm 0$ & $2 \pm 0$ & $3 \pm 0.5$ & $2 \pm 0$ \\
\hline & ARI & 0.5 & 0.5 & 0.3 & 0.1 \\
\hline & NVI & 0.6 & 0.5 & 0.7 & 0.8 \\
\hline \multirow{3}{*}{ Dunn + K-means } & $E[m] \pm s t d[m]$ & $20.4 \pm 2.7$ & $10.7 \pm 2.7$ & $6.7 \pm 5.2$ & $21.1 \pm 2.4$ \\
\hline & ARI & 0.2 & 0.5 & 0.3 & 0.1 \\
\hline & NVI & 0.7 & 0.5 & 0. & 0.8 \\
\hline
\end{tabular}


Table 5.3: Real data set 2. Result is generated from the average of 50 runs.

\begin{tabular}{|c|c|c|c|c|c|}
\hline & & $\begin{array}{c}\text { Yeast } \\
\bar{m}=10, \mathrm{~d}=8, \mathrm{~N}=1484\end{array}$ & $\begin{array}{c}\text { Thyroid } \\
\bar{m}=2, \mathrm{~d}=5, \mathrm{~N}=215\end{array}$ & $\begin{array}{c}\text { WTP } \\
\bar{m}=13, \mathrm{~d}=38, \mathrm{~N}=527\end{array}$ & $\begin{array}{c}\text { Glass } \\
\bar{m}=7, \mathrm{~d}=10, \mathrm{~N}=214\end{array}$ \\
\hline \multirow{3}{*}{$\mathrm{k}-\mathrm{MACE}$} & $E[m] \pm s t d[m]$ & $3 \pm 0.4$ & $\mathbf{3} \pm \mathbf{0 . 9}$ & $8 \pm 0$ & $5.1 \pm 0.4$ \\
\hline & ARI & NA & NA & NA & 0.2 \\
\hline & NVI & NA & NA & NA & 0.8 \\
\hline \multirow{3}{*}{$\mathrm{k}-\mathrm{MACE}_{\sigma}$} & $E[m] \pm s t d[m]$ & $\mathbf{6} \pm \mathbf{0 . 1}$ & $12.1 \pm 1.8$ & $11 \pm 0$ & $9 \pm 0.9$ \\
\hline & ARI & NA & NA & NA & 0.2 \\
\hline & NVI & NA & NA & NA & 0.8 \\
\hline \multirow{3}{*}{ Smart k - $\mathrm{MACE}_{\sigma}$} & $E[m] \pm s t d[m]$ & $1 \pm 0$ & $3 \pm 0.6$ & $5.8 \pm 0.4$ & $5.1 \pm 0.6$ \\
\hline & ARI & NA & NA & NA & 0.2 \\
\hline & NVI & NA & NA & NA & 0.8 \\
\hline \multirow{3}{*}{ MACE-means } & $E[m] \pm s t d[m]$ & $1 \pm 0$ & $1 \pm 0$ & $19.7 \pm 1.8$ & $1 \pm 0$ \\
\hline & ARI & NA & NA & NA & 0.0 \\
\hline & NVI & NA & NA & NA & 1.0 \\
\hline \multirow{3}{*}{ DIP-means } & $E[m] \pm s t d[m]$ & $1 \pm 0$ & $1 \pm 0$ & NA & $2 \pm 0$ \\
\hline & ARI & NA & NA & $\mathrm{NA}$ & 0.1 \\
\hline & NVI & NA & NA & NA & 0.9 \\
\hline \multirow{3}{*}{ G-means } & $E[m] \pm s t d[m]$ & $101 \pm 0$ & $7 \pm 0$ & NA & $11 \pm 0$ \\
\hline & ARI & NA & $\mathrm{NA}$ & NA & 0.1 \\
\hline & NVI & NA & NA & NA & 0.9 \\
\hline \multirow{3}{*}{$\mathrm{X}$-means } & $E[m] \pm s t d[m]$ & $47 \pm 0$ & $11 \pm 0$ & NA & $41 \pm 0.0$ \\
\hline & ARI & NA & NA & NA & 0.1 \\
\hline & NVI & NA & NA & NA & 0.9 \\
\hline \multirow{3}{*}{ DBSCAN } & $E[m] \pm s t d[m]$ & $3 \pm 0$ & $1 \pm 0$ & $2 \pm 0$ & $2 \pm 0.0$ \\
\hline & ARI & NA & NA & NA & 0.2 \\
\hline & NVI & NA & NA & NA & 0.8 \\
\hline \multirow{3}{*}{$\mathrm{CH}+\mathrm{K}$-means } & $E[m] \pm s t d[m]$ & $2 \pm 0$ & $\mathbf{3} \pm \mathbf{0}$ & $15.9 \pm 2.3$ & $2.1 \pm 0.3$ \\
\hline & ARI & NA & NA & NA & 0.2 \\
\hline & NVI & NA & NA & NA & 0.8 \\
\hline \multirow{3}{*}{ Sil + K-means } & $E[m] \pm s t d[m]$ & $3.9 \pm 1$ & $3.7 \pm 0.5$ & $3 \pm 0$ & $3.8 \pm 0.4$ \\
\hline & ARI & NA & NA & NA & 0.2 \\
\hline & NVI & NA & NA & NA & 0.8 \\
\hline \multirow{3}{*}{$\mathrm{DB}+\mathrm{K}$-means } & $E[m] \pm s t d[m]$ & $7.1 \pm 3.1$ & $4.2 \pm 0.8$ & $3 \pm 0$ & $9 \pm 4.4$ \\
\hline & ARI & NA & NA & NA & 0.3 \\
\hline & NVI & NA & NA & NA & 0.7 \\
\hline \multirow{3}{*}{ gap + K-means } & $E[m] \pm s t d[m]$ & $20.1 \pm 0.9$ & $18.7 \pm 1.5$ & $15 . \pm 0.5$ & $16.3 \pm 0.7$ \\
\hline & ARI & NA & $\mathrm{NA}$ & NA & 0.2 \\
\hline & NVI & NA & NA & NA & 0.7 \\
\hline \multirow{3}{*}{ Xie-Beni + K-means } & $E[m] \pm s t d[m]$ & $1 \pm 0$ & $3 \pm 0$ & $3 \pm 0$ & $4 \pm 0$ \\
\hline & ARI & NA & NA & NA & 0.3 \\
\hline & NVI & NA & NA & NA & 0.7 \\
\hline \multirow{3}{*}{ Dunn + K-means } & $E[m] \pm s t d[m]$ & $16.7 \pm 4.4$ & $4 \pm 0$ & $2 \pm 0$ & $4 \pm 0$ \\
\hline & ARI & NA & $\mathrm{NA}$ & NA & 0.3 \\
\hline & NVI & NA & NA & NA & 0.7 \\
\hline
\end{tabular}


Table 5.4: Real data set 3. Result is generated from the average of 50 runs.

\begin{tabular}{|c|c|c|c|c|c|}
\hline & & $\begin{array}{c}\text { Abalone } \\
\bar{m}=29, \mathrm{~d}=8, \mathrm{~N}=4177\end{array}$ & $\begin{array}{c}\text { Breast } \\
\bar{m}=2, \mathrm{~d}=9, \mathrm{~N}=698\end{array}$ & $\begin{array}{c}\text { Soybean } \\
\bar{m}=19, \mathrm{~d}=35, \mathrm{~N}=307\end{array}$ & $\begin{array}{c}\text { WDBC } \\
\bar{m}=2, \mathrm{~d}=32, \mathrm{~N}=569\end{array}$ \\
\hline \multirow{2}{*}{$\mathrm{k}-\mathrm{MACE}$} & $E[m] \pm s t d[m]$ & $2.9 \pm 1.3$ & $2 \pm 0$ & $12.6 \pm 2.4$ & $\mathbf{2} \pm \mathbf{0}$ \\
\hline & $\begin{array}{l}\text { ARI } \\
\text { NVI }\end{array}$ & $\begin{array}{l}0.1 \\
0.9\end{array}$ & $\begin{array}{l}\text { NA } \\
\text { NA }\end{array}$ & $\begin{array}{l}0.4 \\
0.5\end{array}$ & $\begin{array}{l}0.5 \\
0.7\end{array}$ \\
\hline \multirow{3}{*}{$\mathrm{k}-\mathrm{MACE}_{\sigma}$} & $E[m] \pm s t d[m]$ & $22.8 \pm 0.4$ & $3 \pm 0$ & $12.6 \pm 2.9$ & $7 \pm 0$ \\
\hline & ARI & 0.1 & NA & 0.4 & 0.3 \\
\hline & NVI & 0.9 & NA & 0.0 & 0.8 \\
\hline \multirow{3}{*}{ Smart k - $\mathrm{MACE}_{\sigma}$} & $E[m] \pm s t d[m]$ & $7.0 \pm 0.0$ & $2 \pm \mathbf{0}$ & $12.4 \pm 1.2$ & $3.8 \pm 0.8$ \\
\hline & ARI & 0.1 & NA & 0.0 & 0.4 \\
\hline & NVI & 0.9 & NA & 1 & 0.7 \\
\hline \multirow{3}{*}{ MACE-means } & $E[m] \pm s t d[m]$ & $9 \pm 0$ & $1 \pm 0$ & $1 \pm 0$ & $7.2 \pm 2.1$ \\
\hline & ARI & 0.1 & NA & 0.0 & 0.3 \\
\hline & NVI & 0.9 & NA & 1.0 & 0.8 \\
\hline \multirow{3}{*}{ DIP-means } & $E[m] \pm s t d[m]$ & NA & $12 \pm 0$ & $3 \pm 0$ & $1 \pm 0$ \\
\hline & ARI & NA & $\mathrm{NA}$ & 0 & 0 \\
\hline & NVI & NA & NA & 1 & 1 \\
\hline \multirow{3}{*}{ G-means } & $E[m] \pm s t d[m]$ & NA & $96 \pm 0$ & $16 \pm 0$ & $14 \pm 0$ \\
\hline & ARI & NA & NA & 0.3 & 0.2 \\
\hline & NVI & NA & NA & 0.8 & 0.7 \\
\hline \multirow{3}{*}{ X-means } & $E[m] \pm s t d[m]$ & NA & $104 \pm 0$ & $45 \pm 0$ & $129 \pm 0.0$ \\
\hline & ARI & NA & NA & 0.2 & 0.1 \\
\hline & NVI & NA & NA & 0.7 & 0.9 \\
\hline \multirow{3}{*}{ DBSCAN } & $E[m] \pm s t d[m]$ & $4.0 \pm 0.0$ & $11 \pm 0$ & $4.0 \pm 0.0$ & $1 \pm 0$ \\
\hline & ARI & 0.1 & NA & 0.1 & 0.0 \\
\hline & NVI & 0.9 & NA & 0.5 & 1.0 \\
\hline \multirow{3}{*}{$\mathrm{CH}+\mathrm{K}$-means } & $E[m] \pm s t d[m]$ & $12.9 \pm 0.3$ & $\mathbf{2} \pm \mathbf{0}$ & $12.9 \pm 0.3$ & $11.1 \pm 1.0$ \\
\hline & ARI & 0.1 & NA & 0.1 & 0.2 \\
\hline & NVI & 0.9 & NA & 0.8 & 0.8 \\
\hline \multirow{3}{*}{ Sil + K-means } & $E[m] \pm s t d[m]$ & $5 \pm 0$ & $\mathbf{2} \pm \mathbf{0}$ & $15 \pm 4.1$ & $2.8 \pm 0.4$ \\
\hline & ARI & 0.1 & NA & 0.4 & 0.5 \\
\hline & NVI & 0.9 & NA & 0.5 & 0.7 \\
\hline \multirow{3}{*}{$\mathrm{DB}+\mathrm{K}$-means } & $E[m] \pm s t d[m]$ & $9.7 \pm 0.5$ & $\mathbf{2} \pm \mathbf{0}$ & $15.7 \pm 3.8$ & $\mathbf{2} \pm \mathbf{0}$ \\
\hline & ARI & 0.1 & NA & 0.4 & 0.5 \\
\hline & NVI & 0.9 & NA & 0.4 & 0.7 \\
\hline \multirow{3}{*}{ gap + K-means } & $E[m] \pm s t d[m]$ & $13.0 \pm 0.2$ & $10.42 \pm 0.7$ & $27.6 \pm 1.2$ & $10.5 \pm 1.3$ \\
\hline & ARI & 0.1 & NA & 0.4 & 0.2 \\
\hline & NVI & 0.9 & NA & 0.4 & 0.8 \\
\hline \multirow{3}{*}{ Xie-Beni + K-means } & $E[m] \pm s t d[m]$ & NA & $2 \pm 0$ & $3 \pm 0.5$ & $2 \pm 0$ \\
\hline & ARI & NA & 0 & 0.1 & 0.5 \\
\hline & NVI & NA & 1 & 0.8 & 0.7 \\
\hline \multirow{3}{*}{ Dunn + K-means } & $E[m] \pm s t d[m]$ & NA & $2 \pm 0$ & $3 \pm 5.2$ & $20.3 \pm 2.5$ \\
\hline & ARI & NA & 0 & 0.1 & 0.1 \\
\hline & NVI & NA & 1 & 0.8 & 0.8 \\
\hline
\end{tabular}




\section{Chapter 6}

\section{Conclusion and Future Work}

In this thesis, we tackle the problem of estimating the correct number of clusters. The number of clusters is one of the parameters that is required in partitional clustering methods. This parameter is difficult to be intuitively determined beforehand and is very critical to the clustering result. A brief review of some of exisiting methods that attempt to estimate the number of clusters is provided in Chapter 2. Signal denoising scheme that inspired $\mathrm{k}-\mathrm{MACE}$ was also reviewed.

In Chapter 3, $\mathrm{k}$ - MACE algorithm is proposed as a way to estimate the correct number of clusters and is used as a wrapper to k-means clustering. Similar to other validity index method, we define an objective function which is the ACE ACE is a function of the $m$ which is the number of clusters fed into k-means. By minimizing ACE, the estimate of the correct cluster is found. The direct calculation of ACE is not possible in real life since it requires the knowledge of the cluster's true center. However, we have introduced a way to estimate the probabilistic bounds ACE using the available data error. This method is one of the main contributions of this work. One of the biggest limitations of MACE means is that it is designed to work only for data whose clusters has the same 
variance. A modification of $\mathrm{k}-\mathrm{MACE}$ is proposed to alleviate such limitations.

In Chapter 4, we discussed a special case of $\mathrm{k}-\mathrm{MACE}$ and that is when data set is composed of IID clusters with uniform variance, denoted by $\mathrm{k}-\mathrm{MACE}_{\sigma}$. The advantage of this is that it makes the calculation of ACE's bounds simpler thus reducing the complexity.k $-\mathrm{MACE}_{\sigma}$ is still proven to be advantageous over MACE-means although both algorithms are based on the same assumption. The reason for this is that we introduced the bounds on ACE's estimation. MACE means on the other hand, only uses the expectation of ACE. $\mathrm{k}-\mathrm{MACE}_{\sigma}$ when applied to a data set whose clusters are not IID results into overestimation of number of clusters. We proposed a way of detecting this error by checking unimodality of touching clusters. If such error is detected, then assumption that all clusters are IID with the same variance is not applicable and therefore, $\mathrm{k}-\mathrm{MACE}$ is preferable instead of $\mathrm{k}-\mathrm{MACE}_{\sigma}$.

In Chapter 5, we present the simulation result. For synthetic data set $\mathrm{k}-\mathrm{MACE}$ shows superiority when dealing with clusters that has general Gaussian distribution as $\mathrm{k}$ - MACE provided consistent and accurate result even for the case where Gaussian clusters are overlapping. For IID clusters, it was $\mathrm{k}-\mathrm{MACE}_{\sigma}$ that came on top especially when dealing with D31 data set that is compose of overlapping IID clusters. $\mathrm{k}-\mathrm{MACE}_{\sigma}$ also provided accurate and consistent estimate of CNC for IID setting. For real life data, on average, k-MACE performs the best.

In $\mathrm{k}-\mathrm{MACE}$, the covariance of the clusters is estimated using clustering results obtained from calculation of data error. Any error on the m-clustering step will get carried over towards estimation of true cluster covariance which will in turn, affect the estimation of ACE. An example of this is shown for the case when $\mathrm{k}$ - MACE is applied on D31 data set. This being said,one interesting avenue of research is to find a way to obtain a more accurate estimate of the cluster covariance that is independent of the 
clustering result. This will lessen the dependency of the $\mathrm{k}$ - MACE on $m$-clustering result that can improve robustness of the algorithm. Also, by being able to estimate true cluster covariance independently, it will be possible for ACE to be used as a validity index. 
Appendices 



\section{Appendix A}

\section{Average Central Error}

The total central error of cluster $C_{m j}$ is defined by

$$
\begin{gathered}
Z_{s_{m j}}=\left\|\bar{c}_{x_{m j}}-c_{m j}\right\|_{F}^{2} \\
Z_{s_{m j}}=\left\|I \bar{c}_{x_{m j}}-B_{m j} x_{m j}\right\|_{F}^{2}
\end{gathered}
$$

$B_{m j}$ is an averaging matrix that is the following

$$
B_{m j}=\left[\begin{array}{ccc}
\frac{1}{n_{m j}} & \cdots & \frac{1}{n_{m j}} \\
\frac{1}{n_{m j}} & \frac{1}{n_{m j}} & \cdots \\
\cdots & \cdots & \cdots \\
\frac{1}{n_{m j}} & \cdots & \frac{1}{n_{m j}}
\end{array}\right]
$$

where $n_{m j}$ is the number of elements in cluster $C_{m j}$.

The set $x_{m j}$ is formed by taking all elements $x_{i} \in\left(x^{N}\right)$ that belongs to cluster $C_{m j}$.

Making $x_{m j}^{i}=\bar{c}_{x_{m j}^{i}}+\bar{W}_{x_{m j}^{i}}$ and $\bar{W}_{x_{m j}^{i}}=\hat{W}_{x_{m j}^{i}} Q_{x_{m j}^{i}}$, where $\hat{W}_{x_{m j}^{i}}$ is an independent 
Gaussian Random vector and $Q_{x_{m j}^{i}}$ the eigenvector of the $\bar{W}_{x_{m j}^{i}}$ 's covariance matrix,

$$
\begin{array}{r}
Z_{s_{m j}}=\left\|\left(I-B_{m j}\right) \bar{c}_{x_{m j}}-B_{m j} \hat{W}_{x_{m j}} Q_{x_{m j}}\right\|_{F}^{2} \\
Z_{s_{m j}}=\left\|A_{m j} \bar{c}_{x_{m j}}-B_{m j} \hat{W}_{x_{m j}} Q_{x_{m j}}\right\|_{F}^{2}
\end{array}
$$

since $A_{m j}^{T} B_{m j}=0$ and $\left\|Q_{x_{m j}}\right\|_{F}^{2}=1$, we get

$$
\begin{gathered}
Z_{s_{m j}}=\left\|A_{m j} \bar{c}_{x_{m j}}\right\|_{F}^{2}+\left\|B_{m j} \hat{W}_{x_{m j}}\right\|_{F}^{2} \\
\left\|B_{m j} \hat{W}_{x_{m j}}\right\|_{F}^{2}=\frac{1}{n_{m j}} \sum_{i=1}^{n_{m j}} \hat{W}_{x_{m j}^{i}} \hat{W}_{x_{m j}^{i}}^{T}+\frac{1}{n_{m j}} \sum_{i \neq k}^{n_{m j}} \hat{W}_{x_{m j}^{i}} \hat{W}_{x_{m j}^{k}}^{T} \\
Z_{s_{m j}}=\left\|A_{m j} \bar{c}_{x_{m j}}\right\|_{F}^{2}+\frac{1}{n_{m j}} \sum_{i=1}^{n_{m j}} \hat{W}_{x_{m j}^{i}} \hat{W}_{x_{m j}^{i}}^{T}+\frac{1}{n_{m j}} \sum_{i \neq k}^{n_{m j}} \hat{W}_{x_{m j}^{i}} \hat{W}_{x_{m j}^{k}}^{T}
\end{gathered}
$$

From (A.7), the argument inside the second summation term can be expanded as follows:

$$
\begin{aligned}
& \hat{W}_{x_{m j}^{i}} \hat{W}_{x_{m j}^{i}}^{T}=\left[\hat{W}_{x_{m j}^{i}}^{1} \quad \hat{W}_{x_{m j}^{i}}^{2} \ldots \hat{W}_{x_{m j}^{i}}^{d}\right]\left[\hat{W}_{x_{m j}^{i}}^{1} \quad \hat{W}_{x_{m j}^{i}}^{2} \ldots \hat{W}_{x_{m j}^{i}}^{d}\right]^{T} \\
& \hat{W}_{x_{m j}^{i}} \hat{W}_{x_{m j}^{i}}^{T}=\left(\hat{W}_{x_{m j}^{i}}^{1}\right)^{2}+\left(\hat{W}_{x_{m j}^{i}}^{2}\right)^{2}+\ldots+\left(\hat{W}_{x_{m j}^{i}}^{d}\right)^{2}
\end{aligned}
$$

where $\hat{W}_{x_{m j}^{i}}^{d^{\prime}}$ refers to the variation of element $i$ of the cluster $C_{m j}$ on the $d^{\prime}$ component. Applying equation A.8), the second term on equation A.7 becomes a Chi-square random variable with degrees of freedom $d o f=n_{m j} * d$ 


\section{A.1 Expected Value of ACE}

$Z_{s_{m j}}$ is a function of the random vector $\hat{W}_{x_{m j}^{i}}$. The first term of $Z_{s_{m j}}$ and the last term has an expected value of zero. The second term is chi-square random variable with a non-zero mean. The overall expected value of $Z_{s_{m j}}$ is therefore given by

$$
E\left[Z_{s_{m j}}\right]=\left\|A_{m j} \bar{c}_{x_{m j}}\right\|_{F}^{2}+\frac{1}{n_{m j}} \sum_{i=1}^{n_{m j}} \operatorname{tr}\left(\bar{\wedge}_{x_{m j}^{i}}\right)
$$

where $\bar{\Lambda}_{x_{m j}^{i}}$ is the diagonal matrix that represents the covariance of the independent random vector $\bar{W}_{x_{m j}^{i}}$.

\section{A.2 Variance of $\mathrm{ACE}$}

The first term of equation A.7 is a constant thus, it has a variance of zero. The variance of the two following terms are given below:

$$
\begin{gathered}
\operatorname{Var}\left[\frac{1}{n_{m j}} \sum_{i=1}^{n_{m j}} \hat{W}_{x_{m j}^{i}} \hat{W}_{x_{m j}^{i}}^{T}\right]=\frac{2}{n_{m j}^{2}} \sum_{i=1}^{n_{m j}} \operatorname{tr}\left(\bar{\wedge}_{x_{m j}^{i}}{ }^{2}\right) \\
\operatorname{Var}\left[\frac{1}{n_{m j}} \sum_{i \neq k}^{n_{m j}} \hat{W}_{x_{m j}^{i}} \hat{W}_{x_{m j}^{k}}^{T}\right]=\frac{2}{n_{m j}^{2}} \sum_{i \neq k}^{n_{m j}} \operatorname{tr}\left({\bigwedge_{x_{m j}^{i}}}_{{ }_{i}} \bar{\wedge}_{m j}^{k}\right)
\end{gathered}
$$

The covariance between the second and term term of $Z_{s_{m j}}$ in A.7 is 0 . Therefore, the variance of $Z_{s_{m j}}$ is given by the following:

$$
\operatorname{Var}\left[Z_{s_{m j}}\right]=\frac{2}{n_{m j}^{2}} \sum_{i=1}^{n_{m j}} \operatorname{tr}\left(\left({\bigwedge_{x_{m j}^{i}}}^{2}\right)+\frac{2}{n_{m j}{ }^{2}} \sum_{i \neq k}^{n_{m j}} \operatorname{tr}\left(\bar{\wedge}_{x_{m j}^{i}} \bar{\wedge}_{x_{m j}^{k}}\right)\right.
$$




\section{A.3 Average Central Error When $\bar{\Sigma}_{X_{i}}=\sigma_{w}^{2} I_{d x d}$}

Assuming that the variations on all cluster is $I I D$ with variance $\sigma_{w}^{2}$, we have

$$
W_{x_{m j}^{i}} \sim \mathcal{N}\left(0, \sigma_{w}^{2} I_{d x d}\right), i=1,2, \ldots N
$$

It follows that

$$
\begin{gathered}
\operatorname{tr}\left(\bar{\wedge}_{x_{m j}^{i}}\right)=d \sigma_{w}^{2} \\
E\left[Z_{s_{m j}}\right]=\left\|A_{m j} \bar{c}_{x_{m j}}\right\|_{F}^{2}+d \sigma_{w}^{2} \\
\operatorname{Var}\left[Z_{s_{m j}}\right]=\frac{2 d}{n_{m j}^{2}}\left(n_{m j} \sigma_{w}^{4}\right)+\frac{2}{n_{m j}^{2}}\left(n_{m j}\right)\left(n_{m j}-1\right) d \sigma_{w}^{4} \\
\operatorname{Var}\left[Z_{s_{m j}}\right]=2 d \sigma_{w}^{4}
\end{gathered}
$$

Taking equations A.15 and A.16) to solve for expected value and variance of the overall average central error, we have

$$
E\left[Z_{s_{m}}\right]=\frac{1}{N} \sum_{j=1}^{m}\left\|A_{m j} \bar{c}_{x_{m j}}\right\|_{F}^{2}+\frac{d m \sigma_{w}^{2}}{N}
$$

The covariance between $Z_{s_{m j}}$ 's are equal to zero. (i.e $\operatorname{Cov}\left[Z_{s m j}, Z_{s m k}\right]_{j \neq k}=0$

$$
\operatorname{Var}\left[Z_{s_{m}}\right]=\frac{2 m d \sigma_{w}^{4}}{N^{2}}
$$

The upperbound of $Z_{s_{m}}$ can be calculated directly under the assumption that $W_{x_{i}} \sim$ 
$\mathcal{N}\left(0, \sigma_{w}^{2} I_{d x d}\right)$, for $i=1,2, \ldots N$. Letting $\beta_{n}=N$

$$
\begin{gathered}
\overline{Z_{s_{m}}}=E\left[Z_{s_{m}}\right]+\beta_{n} \sqrt{\operatorname{Var}\left[Z_{s_{M}}\right]} \\
\overline{Z_{s_{m}}}=\frac{1}{N} \sum_{j=1}^{m}\left\|A_{m j} \bar{c}_{x_{m j}}\right\|_{F}^{2}+\frac{d m \sigma_{w}^{2}}{N}+N \sqrt{\frac{2 m d \sigma_{w}^{4}}{N^{2}}}
\end{gathered}
$$

and finally, the upperbound of $Z_{s_{m}}$ is given by

$$
\overline{Z_{s_{m}}}=\frac{1}{N} \sum_{j=1}^{m}\left\|A_{m j} \bar{c}_{x_{m j}}\right\|_{F}^{2}+\frac{d m \sigma_{w}^{2}}{N}+\sigma_{w}^{2} \sqrt{2 m d}
$$

Similarly, the lower bound of $Z_{s_{m}}$ is given by

$$
\begin{gathered}
\underline{Z_{s_{m}}}=E\left[Z_{s_{m}}\right]-\beta_{n} \sqrt{\operatorname{Var}\left[Z_{s_{M}}\right]} \\
\underline{Z_{s_{m}}}=\frac{1}{N} \sum_{j=1}^{m}\left\|A_{m j} \bar{c}_{x_{m j}}\right\|_{F}^{2}+\frac{d m \sigma_{w}^{2}}{N}-N \sqrt{\frac{2 m d \sigma_{w}^{4}}{N^{2}}} \\
\underline{Z_{s_{m}}}=\frac{1}{N} \sum_{j=1}^{m}\left\|A_{m j} \bar{c}_{x_{m j}}\right\|_{F}^{2}+\frac{d m \sigma_{w}^{2}}{N}-\sigma_{w}^{2} \sqrt{2 m d}
\end{gathered}
$$




\section{Appendix B}

\section{Data Error}

The defenition of cluster compactness is given by

$$
\begin{gathered}
Y_{s_{m j}}=\left\|x_{m j}-c_{m j}\right\|_{F}^{2} \\
Y_{s_{m j}}=\left\|x_{m j}-B_{m j} x_{m j}\right\|_{F}^{2} \\
Y_{s_{m j}}=\left\|A_{m j} x_{m j}\right\|_{F}^{2}
\end{gathered}
$$

$$
\begin{aligned}
& \text { Making } x_{m j}^{i}=\bar{c}_{x_{m j}^{i}}+\hat{W}_{x_{m j}^{i}} Q_{x_{m j}^{i}} \text { we get } \\
& \qquad Y_{s_{m j}}=\left\|\bar{c}_{x_{m j}}+\hat{W}_{x_{m j}} Q_{x_{m j}}\right\|_{F}^{2}
\end{aligned}
$$




$$
\begin{array}{r}
Y_{s_{m j}}=\left\|A_{m j} \bar{c}_{x_{m j}}\right\|_{F}^{2}+\left\|A_{m j} \hat{W}_{x_{m j}}\right\|_{F}^{2}+ \\
\frac{2\left(n_{m j}-1\right)}{n_{m j}} \sum_{i=1}^{n_{m j}} \bar{c}_{x_{m j}^{i}} \hat{W}_{x_{m j}^{i}}^{T}-\frac{2}{n_{m j}} \sum_{i \neq k}^{n_{m j}}\left(\bar{c}_{x_{m j}^{i}} \hat{W}_{x_{m j}^{k}}^{T}+\bar{c}_{x_{m j}^{k}} \hat{W}_{x_{m j}^{i}}^{T}\right)
\end{array}
$$

Finally,

$$
\begin{aligned}
Y_{s_{m j}}=\left\|A_{m j} \bar{c}_{x_{m j}}\right\|_{F}^{2}+ & \frac{n_{m j}-1}{n_{m j}} \sum_{i=1}^{n_{m j}} \bar{W}_{x_{m j}^{i}} \bar{W}_{x_{m j}^{i}}^{T}-\frac{1}{n_{m j}} \sum_{i \neq k}^{n_{m j}} \bar{W}_{x_{m j}^{i}} \bar{W}_{x_{m j}^{k}}^{T} \\
& 2 \sum_{i=1}^{n_{m j}} \bar{c}_{x_{m j}^{i}} \bar{W}_{x_{m j}^{i}}^{T}-\frac{2}{n_{m j}} \sum_{d^{\prime}=1}^{d}\left(\sum_{i=1}^{n_{m j}} \bar{W}_{x_{m j}^{i}}^{d^{\prime}} \sum_{i=1}^{n_{m j}} \bar{c}_{x_{m j}^{i}}^{d^{\prime}}\right)
\end{aligned}
$$

\section{B.1 Expected Value of Data Error}

The expected value of $\hat{W}_{x_{m j}^{i}}$ is zero, making terms 4 and 5 of B.6 expectation to b also zero. The 3rd term's expectation is also zero due to independence between random variables $\hat{W}_{x_{m j}^{i}}$ and $\hat{W}_{x_{m j}^{k}}(i \neq k)$. The expected value of $Y_{s_{m j}}$ is given by

$$
E\left[Y_{s_{m j}}\right]=\left\|A_{m j} \bar{c}_{x_{m j}}\right\|_{F}^{2}+\frac{\left(n_{m j}-1\right)}{n_{m j}} \sum_{i=1}^{n_{m j}} \operatorname{tr}\left({\overline{\bigwedge_{x_{m j}^{i}}}}\right)
$$

\section{B.2 Variance of Data Error}

The first term of equation $(\overline{B .6})$ is a constant thus, it has a variance of zero. The variance of the four following terms are given below:

$$
\operatorname{Var}\left[\frac{n_{m j}-1}{n_{m j}} \sum_{i=1}^{n_{m j}} \bar{W}_{x_{m j}^{i}} \bar{W}_{x_{m j}^{i}}^{T}\right]=\frac{2\left(n_{m j}-1\right)^{2}}{n_{m j}^{2}} \sum_{i=1}^{n_{m j}} \operatorname{tr}\left(\left(\bar{\wedge}_{x_{m j}^{i}}\right)^{2}\right)
$$




$$
\begin{array}{r}
\operatorname{Var}\left[-\frac{1}{n_{m j}} \sum_{i \neq k}^{n_{m j}} \bar{W}_{x_{m j}^{i}} \bar{W}_{x_{m j}^{k}}^{T}\right]=\frac{2}{n_{m j}^{2}} \sum_{i \neq k}^{n_{m j}} \operatorname{tr}\left(\bar{\wedge}_{x_{m j}^{i}} \bar{\lambda}_{x_{m j}^{k}}\right) \\
\operatorname{Var}\left[2 \sum_{i=1}^{n_{m j}} \bar{c}_{x_{m j}^{i}} \bar{W}_{x_{m j}^{i}}^{T}\right]=\frac{4}{n_{m j} d} \sum_{i=1}^{n_{m j}} \operatorname{tr}\left(\bar{\wedge}_{x_{m j}^{i}}\right) \sum_{i=1}^{n_{m j}} \bar{c}_{x_{m j}^{i}} \bar{c}_{x_{m j}^{i}}^{T} \\
\operatorname{Var}\left[-\frac{2}{n_{m j}} \sum_{d^{\prime}=1}^{d}\left(\sum_{i=1}^{n_{m j}} \bar{W}_{x_{m j}^{i}}^{d^{\prime}} \sum_{i=1}^{n_{m j}} \bar{c}_{x_{m j}^{i}}^{d^{\prime}}\right)\right]= \\
\frac{4}{n_{m j}^{2} d} \sum_{i=1}^{n_{m j}} \operatorname{tr}\left(\bar{\wedge}_{x_{m j}^{i}}\right)\left(\sum_{i=1}^{n_{m j}} \bar{c}_{x_{m j}^{i}} \bar{c}_{x_{m j}^{i}}^{T}+\sum_{i \neq k}^{n_{m j}} \bar{c}_{x_{m j}^{i}} \bar{c}_{x_{m j}^{k}}^{T}\right)
\end{array}
$$

The covariance between terms 4 and 5 of equation (B.6) is given below:

$$
\begin{array}{r}
2 \operatorname{Cov}\left[\left[2 \sum_{i=1}^{n_{m j}} \bar{c}_{x_{m j}^{i}} \bar{W}_{x_{m j}^{i}}^{T},-\frac{2}{n_{m j}} \sum_{d^{\prime}=1}^{d}\left(\sum_{i=1}^{n_{m j}} \bar{W}_{x_{m j}^{i}}^{d^{\prime}} \sum_{i=1}^{n_{m j}} \bar{c}_{x_{m j}^{i}}^{d^{\prime}}\right)\right]=\right. \\
\frac{-8}{n_{m j}^{2} d} \sum_{i=1}^{n_{m j}} \operatorname{tr}\left(\bar{\wedge}_{x_{m j}^{i}}\right)\left(\sum_{i=1}^{n_{m j}} \bar{c}_{x_{m j}^{i}} \bar{c}_{x_{m j}^{i}}^{T}+\sum_{i \neq k}^{n_{m j}} \bar{c}_{x_{m j}^{i}} \bar{c}_{x_{m j}^{k}}^{T}\right)
\end{array}
$$

while the rest of the covariance between terms $2-5$ of $(\overline{B .6})$ is zero.

Note that

$$
\left\|A_{m j} \bar{c}_{x_{m j}}\right\|_{F}^{2}=\frac{n_{m j}-1}{n_{m j}} \sum_{i=1}^{n_{m j}} \bar{c}_{x_{m j}^{i}} \bar{c}_{x_{m j}^{i}}^{T}-\frac{1}{n_{m j}} \sum_{i \neq k}^{n_{m j}} \bar{c}_{x_{m j}^{i}} \bar{c}_{x_{m j}^{k}}^{T}
$$

Adding equations B.8 B.12 and using equation B.13, the variance of $Y_{s_{m_{j}}}$ is given 
below

$$
\begin{array}{r}
\operatorname{Var}\left[Y_{s_{m j}}\right]=\frac{2\left(n_{m j}-1\right)^{2}}{n_{m j}^{2}} \sum_{i=1}^{n_{m j}} \operatorname{tr}\left(\left(\bar{\wedge}_{x_{m j}^{i}}\right)^{2}\right)+\frac{1}{n_{m j}^{2}} \sum_{i \neq k}^{n_{m j}} \operatorname{tr}\left(\bar{\wedge}_{x_{m j}^{i}} \bar{\wedge}_{x_{m j}^{k}}\right) \\
\frac{4}{d n_{m j}}\left\|A_{m j} \bar{c}_{x_{m j}}\right\|_{F}^{2} \sum_{i=1}^{n_{m j}} \operatorname{tr}\left(\bar{\wedge}_{x_{m j}^{i}}\right)
\end{array}
$$

\section{B.3 Average Central Error When $\bar{\Sigma}_{\mathbf{X}_{\mathrm{i}}}=\sigma_{\mathrm{w}}^{2} \mathbf{I}_{\mathrm{dxd}}$}

Assuming that the variations on all cluster is $I I D$ with variance $\sigma_{w}^{2}$, we have

$$
\begin{gathered}
E\left[Y_{s_{m j}}\right]=\left\|A_{m j} \bar{c}_{x_{m j}}\right\|_{F}^{2}+d\left(n_{m j}-1\right) \sigma_{w}^{2} \\
\operatorname{Var}\left[Y_{s_{m j}}\right]=4 \sigma_{w}^{2}\left\|A_{m j} \bar{c}_{x_{m j}}\right\|_{F}^{2}+2 d\left(n_{m j}-1\right) \sigma_{w}^{4}
\end{gathered}
$$

Finally taking equations $(\mathrm{B} .15)-(\mathrm{B} .16)$ to solve for overall cluster compactness' expected value and variance,

$$
E\left[Y_{s_{m}}\right]=\frac{1}{N} \sum_{j=1}^{m}\left\|A_{m j} \bar{c}_{x_{m j}}\right\|_{F}^{2}+\frac{d(N-m) \sigma_{w}^{2}}{N}
$$

The covariance between $Y_{s_{m j}}$ 's are equal to zero. (i.e $\operatorname{Cov}\left[Y_{s m j}, Y_{s m k}\right]_{j \neq k}=0$ ).

$$
\operatorname{Var}\left[Y_{s_{m}}\right]=\frac{4 \sigma_{w}^{2}}{N^{2}} \sum_{j=1}^{m}\left\|A_{m j} \bar{c}_{x_{m j}}\right\|_{F}^{2}+\frac{2 d(N-m) \sigma_{w}^{4}}{N}
$$

The following inequality lets us solve the bounds of $\frac{1}{N} \sum_{j=1}^{m}\left\|A_{m j} \bar{c}_{x_{m j}}\right\|_{F}^{2}$

$$
E\left[Y_{s_{m}}\right]-\alpha_{N} \sqrt{\operatorname{Var}\left[Y_{s_{m}}\right]} \leq y_{s_{m}}
$$


Plugging-in equations (4.8) and (4.9) to equation $(\mathrm{B} .19)$ and solving for the boundary, we get

$$
\begin{gathered}
\frac{1}{N} \sum_{j=1}^{m}\left\|A_{m j} \bar{c}_{x_{m j}}\right\|_{F}^{2}+\frac{d(N-m) \sigma_{w}^{2}}{N}-y_{s_{m}}= \\
\alpha_{N} \sqrt{\frac{4 \sigma_{w}^{2}}{N^{2}} \sum_{j=1}^{m}\left\|A_{m j} \bar{c}_{x_{m j}}\right\|_{F}^{2}+\frac{2 d(N-m) \sigma_{w}^{4}}{N}}
\end{gathered}
$$

letting $\frac{1}{N} \sum_{j=1}^{m}\left\|A_{m j} \bar{c}_{x_{m j}}\right\|_{F}^{2}=m_{l}, m_{w}=\frac{d(N-m) \sigma_{w}^{2}}{N}$, we get

$$
m_{l}+m_{w}-y_{s_{m}}=\alpha \sqrt{\frac{4 \sigma_{w}^{2}}{N} m_{l}+\frac{2 \sigma_{w}^{2}}{N} m_{w}}
$$

Squaring both sides

$$
\left(m_{l}+m_{w}-y_{s_{m}}\right)^{2}=\alpha^{2}\left(\frac{4 \sigma_{w}^{2}}{N} m_{l}+\frac{2 \sigma_{w}^{2}}{N} m_{w}\right)
$$

Equation (B.22) is a quadratic equations in terms of $m_{l}$. Solving for the roots of $m_{l}$ can give us both the lowerbound and the upperbound with the higher root of $m_{l}$ being its upperbound.

$$
\begin{aligned}
& \overline{m_{l}}=y_{s_{m}}-m_{w}+2 \frac{\alpha^{2} \sigma_{w}^{2}}{N}+k_{s m}(\alpha) \\
& \underline{m_{l}}=y_{s_{m}}-m_{w}+2 \frac{\alpha^{2} \sigma_{w}^{2}}{N}-k_{s m}(\alpha)
\end{aligned}
$$

and

$$
k_{s m}(\alpha)=\frac{2 \alpha \sigma_{w}}{\sqrt{N}} \sqrt{\frac{\alpha^{2} \sigma_{w}^{2}}{N}+y_{s_{m}}-\frac{1}{2} m_{w}}
$$





\section{References}

[1] J. Shi, J. Malik. "Normalized cuts and image segmentation." IEEE Transactions on Pattern Analysis and Machine Intelligence, vol.22, pp. 888-905, 2000.

[2] O.J. Oyelade, O.O. Oladipupo, I.C. Obagbuwai. "Application of k-means clustering algorithm for prediction of students' academic performance." International Journal of Computer Science and Information Security, Vol. 7, No. 1, 2010.

[3] X. Wang, J. Garibaldi. "A comparison of fuzzy and non-fuzzyclustering techniques in cancer diagnosis." International conference in compututational intelligence in medecine and healthcare, pp. 250-256, 2005.

[4] P. Chatterjee, P. Milanfar. "Patch-based Near-Optimal Image Denoising." IEEE Transactions on Image Processing, vol. 21, no. 4, pp. 1635-1649, April 2012.

[5] J. Han, M. Kamber. Data Mining: Concepts and Techniques, San Francisco, CA: Morgan-Kaufman, 2000, pp. 444-445.

[6] S. Na, L. Xumin, G. Yong. "Research on k-means Clustering Algorithm: An Improved k-means Clustering Algorithm." Intelligent Information Technology and Security Informatics (IITSI), pp. 63-67, 2010. 
[7] X.Xie, G. Beni. "A validity measure for fuzzy clustering. "IEEE transaction on Pattern Analysis and Machine Intelligence, vol. 8, pp.841-847,1991.

[8] L. Kaufman, P.J. Rousseeuw. Finding groups in data: an introduction to cluster analysis, New York, NY: Wiley, 1990.

[9] J.C. Dunn. "Well separated clusters and optimal fuzzy partitions." Journal of Cybernetics, vol.4, no.1, pp. 95-104,1974.

[10] T. Caliski, J. Harabasz. "A dendrite method for cluster analysis." Communications in Statistics, vol. 3, no.1, pp. 1-27, 1974.

[11] D. L. Davies, D. W. Bouldin. "A cluster separation measure." IEEE Transactions on Pattern Analysis and Machine Intelligence, vol. PAMI-1, no. 2, pp. 224 - 227, April 1979.

[12] W.J. Krzanowski, Y.T Lai. "A criterion for determining the number of groups in a data set using sum-of-squares clustering." Biometrics, vol, 44 no.1 pp. 23-34, 1988.

[13] A. Strehl. "Relationship-based clustering and cluster ensemble for high dimensional data mining." PhD thesis. The University of Texas at Austin, May 2002.

[14] Y. Fukuyama, M. Sugeno. "A new method of choosing the number of clusters for the fuzzy c-means method." Proceeding of fifth Fuzzy System Symp., pp. 247 250, 1989.

[15] I. Gath, A. B. Geva. "Unsupervised optimal fuzzy clustering." IEEE transaction on pattern analysis and machine intelligence, vol. 11, no. 7, pp. 773 - 780, Jul. 1989.

[16] M. K. Pakhira, S. Bandyopadhyay, U. Maulik. "Validity index for crisp and fuzzy clusters." Pattern Recognition, vol. 37, pp. 487 - 501, 2004. 
[17] D. Pelleg, A. W. Moore. "X-means: Extending k-means with efficient estimation of the number of clusters." Proceedings of the Seventeenth Internatioanl conferecne on Machine Learning, ICML '00, Morgan Kaufmann Publishers Inc., pp. 727 - 734, 2000

[18] Z. Zhao, S. Guo , Q. Xu , T. Ban. "G-means: a clustering algorithm for intrusion detection." Proceedings of the 15th international conference on Advances in neuroinformation processing, November 2008, pp 563-570

[19] R. E. Kass, L. Wasserman. "A reference Bayesian test for nested hypotheses and its relationship to the Schwarz criterion." Journal of the American Statistical Association, Vol. 90, 1995

[20] T. W. Anderson, D. A. Darling. "Asymptotic theory of certain "goodness-of-fit" criteria based on stochastic processes." Annals of Mathematical Statistics vol. 23, no. 2, pp. 193-212, 1952.

[21] S. Beheshti, M.A. Dahleh. "Noisy Data and Impulse Response Estimation." IEEE Transaction on Signal Processing, Vol. 58, No.2 Feb, 2010.

[22] S. Beheshti, M.A. Dahleh. "A New Information-Theoritc Approach to Signal Denoising and Best Basis Selection." IEEE Transaction on Signal Processing, Vol. 53, No.10 Feb, 2005.

[23] M. Shahbaba, S. Beheshti. "MACE-means clustering." ELSEVIER Signal Processing, vol. 105, pp. 216-225, 2014.

[24] S. Killip, M. Ziyad, K. Pearce, "What Is an Intracluster Correlation Coefficient? Crucial Concepts for Primary Care Researchers." Annals of Family Medicine, vol.2, no.3, pp. 204208, 2004. 
[25] A. Zimek, E. Schubert, H. P. Kriegel. "A survey on unsupervised outlier detection in high-dimensional numerical data." Statistical Analysis and Data Mining, vol.b5, pp 363 - 387 .

[26] M. Ringner. "What is principal component analysis." Nature Biotechnology, vol. 26, pp303 - 304, 2008

[27] L. Wang, C. Leckie, K. Ramamohanarao, J. Bezdek. "Automatocally Determining the Number of Clusters in Unlabeled Data Sets." IEEE Transactions on Knowledge and Data Engineering, vol 21, No. 3, pp 335-350, 2009

[28] J.C. Bezdek, R. Hathaway. "VAT: A Tool for Visual Assesment of (Cluster) Tendency." Proc. Int'l joint Conf. Neural networks (IJCNN'02), pp. 2225-2230,2002

[29] A. Gionis, H. Mannila, P. Tsaparas. "Clustering aggregation." ACM Transactions on Knowledge Discovery from Data (TKDD), vol.1, no.1, pp. 1-30, 2007.

[30] C.J. Veenman, M.J.T. Reinders, E. Backer. "A maximum variance cluster algorithm." IEEE Trans. Pattern Analysis and Machine Intelligence, vol. 24, no. 9, pp. $1273-1280,2002$ 